\title{
Don't get it or don't spread it: Comparing self-interested versus prosocial motivations for COVID- 19 prevention behaviors
}

\author{
Jillian J. Jordan ${ }^{1 *}$, Erez Yoeli ${ }^{2}$, and David G. Rand ${ }^{2}$ \\ ${ }^{1}$ Harvard Business School \\ ${ }^{2}$ Sloan School of Management, Massachusetts Institute of Technology \\ *To whom correspondence should be addressed: jijordan@hbs.edu
}

\begin{abstract}
COVID-19 prevention behaviors may be seen as self-interested or prosocial. Using American samples from MTurk and Prolific (total $n=6,850$ ), we investigated which framing is more effective - and motivation is stronger - for fostering prevention behavior intentions. We evaluated messaging that emphasized personal, public, or personal and public benefits of prevention. In initial studies (conducted March 14-16, 2020), the Public treatment was more effective than the Personal treatment, and no less effective than the Personal+Public treatment. In additional studies (conducted April 17-30, 2020), all three treatments were similarly effective. Across all these studies, the perceived public threat of coronavirus was also more strongly associated with prevention intentions than the perceived personal threat. Furthermore, people who behaved prosocially in incentivized economic games years before the pandemic had greater prevention intentions. Finally, in a field experiment (conducted December 21-23, 2020), we used our three messaging strategies to motivate contact-tracing app signups $(n=152,556$ newsletter subscribers). The design of this experiment prevents strong causal inference; however, the results provide suggestive evidence that the Personal+Public treatment may have been more effective than the Personal or Public treatment. Together, our results highlight the importance of prosocial motives for COVID-19 prevention.
\end{abstract}




\section{Introduction}

The COVID-19 pandemic has caused millions of deaths worldwide, and continues to pose an enormous global threat. It thus remains essential that people engage in behaviors that help prevent infection (e.g., masking, personal hygiene, and physical distancing) ${ }^{1}$ even after the initial introduction of vaccines ${ }^{2}$. Many preventative behaviors, however, are difficult to adhere to or require substantial personal sacrifices. What motivates people to engage in them?

One reason this question is interesting is that coronavirus prevention behaviors protect both the individuals who engage in them and society at large. Thus, they may be conceptualized as either self-interested actions (that serve to benefit the actor) or as cooperative efforts (that, in addition to benefiting the actor, serve also to benefit others in society). Here we investigate the relative strength of these motivations, and the relative efficacy of these framings, for encouraging prevention behaviors.

One might naturally expect a dominant role of self-interest for coronavirus prevention. According to classical economic theories of decision-making, people care only for their own welfare. This perspective would suggest that self-interest should be the strongest motivator. As Adam Smith famously wrote, "It is not from the benevolence of the butcher, the brewer, or the baker that we expect our dinner, but from their regard to their own interest" 5 .

Yet while the classical economic perspective remains highly influential, behavioral scientists are increasingly aware of the importance of more psychologically informed theories ${ }^{4,6-}$ ${ }^{8}$. Research in psychology and behavioral economics provides clear evidence that people care about the welfare of others and are motivated to cooperate ${ }^{9-13}$, and that people strive to avoid appearing selfish in the eyes of others and are highly sensitive to social norms ${ }^{14-17}$. Thus, psychological research reveals that people have "prosocial motivations" (a term we use to refer to any motivation to promote the welfare of others - including those that may be implicitly or explicitly driven by reputation concerns or the desire to adhere to social norms).

While much of this research - which has focused extensively on behavior in economic games - suggests that people care more about their own welfare than the welfare of others, regard for others seems especially strong in the domain of physically aversive outcomes. Experiments reveal that people sometimes voluntarily undergo painful experiences to relieve the suffering of others ${ }^{18}$. Furthermore, people tend to be more risk-averse when making decisions on behalf of others ${ }^{19}$, including in the context of physical harm ${ }^{20}$. And evidence suggests that when people are tasked with allocating pain between themselves versus others, they tend to behave more generously than when they are tasked with allocating money between themselves versus others $^{21-23}$.

In one such study, people were actually less willing to harm others than to harm themselves ${ }^{24}$. However, other studies have demonstrated that, even in the domain of harm, people prioritize their own outcomes equally or above the outcomes of others ${ }^{21-23}$. Thus, previous basic science research investigating prosociality provides some reason to doubt that self-interested motives are dominant, and self-interested framings are more effective, for coronavirus prevention. Yet it nonetheless provides no clear prediction regarding the relative role of prosociality.

Another stream of applied research has investigated self-interested versus prosocial motives in the context of health behavior (and especially vaccination decisions), and has provided clear evidence that both motives can encourage disease prevention ${ }^{25-29}$. A few studies have directly compared the relative effectiveness of personal versus public framing in 
vaccination appeals, with inconsistent results: some studies found that public framing was ineffective ${ }^{30-32}$, while others provided some mixed evidence for the effectiveness of public framing ${ }^{33,34}$; and a set of field studies investigating handwashing among healthcare professionals found that it was more effective to emphasize patient safety than personal safety ${ }^{35}$. Thus, the literature surrounding disease spread prevention likewise does not make a clear prediction regarding the relative importance of self-interested versus prosocial motivations, and the relative efficacy of self-interested versus prosocial framing, for coronavirus prevention.

To investigate, we measure the influence of three messaging treatments on intentions to engage in COVID-19 prevention behaviors: one that emphasizes personal benefits of prevention (Personal message), one that emphasizes public benefits of prevention (Public message), and one that emphasizes both types of benefits (Personal+Public message). Our Personal message was designed to encourage subjects to simply consider the value of prevention behaviors for themselves, while our Public message was designed to prompt subjects to consider that prevention behaviors can, in addition to conferring personal benefits, also benefit others. Finally, our Personal+Public message was designed to explicitly encourage subjects to consider both types of benefits.

In a first set of studies (Studies 1-2, total $n=2176$ ) conducted early in the pandemic (between March 14 and March 16, 2020, at which time there were under 2000 confirmed U.S. cases), we find that (i) the Public message was more effective than the Personal message, and (ii) the Personal+Public message was no more effective than the Public message. In a second a set of studies (Study 3a-d, total $n=3985$ ) conducted slightly later in the pandemic (between April 17 and April 30, 2020, at which time there were 500,000 to 1,000,000 confirmed U.S. cases), we find that all three messaging strategies were similarly effective.

We also take a correlational approach to investigate the extent to which prosocial versus self-interested motivations predict prevention intentions. Across Studies 1-3, as well as an additional study using a more representative sample (Study 4), we consistently find that the perceived public threat of coronavirus is a stronger predictor of prevention intentions than the perceived personal threat. And in Study 5, by linking data from Studies 1-3 to an external dataset of incentivized economic game decisions, we find that people who behaved prosocially years before the pandemic report greater prevention intentions.

Finally, in Study 6, which we conducted more than half a year later (between December 21 and December 23, 2020), we employ a field experiment to investigate the power of our three aforementioned messaging strategies to motivate people to sign up for a contact tracing app. Randomization in the experiment was imperfect, preventing strong causal inference, but the results provide suggestive evidence that combining self-interested and prosocial framing may have been more effective than self-interested or prosocial framing alone.

Together, our results challenge the hypotheses that self-interested motives are the dominant driver of prevention intentions and self-interested appeals are the most effective messaging strategy. Instead, they suggest that prosociality can play an important role in combatting COVID-19.

\section{Studies 1 and 2}

We begin by describing the method and results of our first set of studies, conducted in the early days of the COVID-19 pandemic reaching the United States. 
For all studies reported in this paper, written informed consent was obtained from all participants, the study was approved by the Massachusetts Institute of Technology Committee on the Use of Humans as Experimental Subjects, and all research was performed in accordance with relevant guidelines and regulations.

\section{Method}

\section{Overview}

On March 14 (Study 1) and March 16 (Study 2), 2020, we conducted two studies online using convenience samples from Amazon Mechanical Turk (Mturk). Although there is a considerable amount of evidence supporting the validity of Mturk samples for social science research $^{36,37}$, Mturk samples are not nationally representative. Most notably, participants from Mturk skew much younger than the national age distribution. Yet we nonetheless see our Mturk samples as representative of an important population. Young people are a frequent target for COVID-19 prevention messaging, because they are perceived as less likely to engage in prevention behaviors (a perception that is consistent with our data from all studies in this paper; see SI Section 4.1).

Studies 1-3 were all pre-registered, and our sample sizes were based on our preregistrations. Furthermore, our analyses adhere closely to our pre-registered analysis plans. We note the substantive exceptions in our main text where relevant, and list all exceptions (as well as links to our pre-registrations) in SI Section 5. We also note that for all studies in this paper, our full materials, raw data, and a script reproducing all analyses are available at https://osf.io/sr4n9/.

Studies 1 and 2 used very similar designs, but differed in a few ways. We begin by describing Study 1, and then describe the ways that Study 2 differed from Study 1.

\section{Design}

In Study 1, we recruited a target of $n=1000$ subjects, and assigned them to one of four experimental conditions, which included a control condition (involving no treatment) and three treatment conditions (Personal, Public, and Personal+Public).

After obtaining consent from all subjects, we presented subjects in our treatment conditions with their assigned treatment. In all three treatment conditions, subjects were presented with (i) some written text and then (ii) a flier, before being directed to complete our set of outcome measures. In contrast, in the control condition, subjects were not presented with a written text or a flier, and began the study by completing our set of outcome measures.

We designed our three treatment conditions to be very similar, but manipulated whether they emphasized the personal, public, or personal and public benefits of coronavirus prevention behaviors. We note that while we see our manipulations (both in Study 1 and throughout this paper) as having high face validity, we did not collect any manipulation check measures to evaluate whether these benefits were successfully communicated to subjects.

\section{Treatments}

In all treatments, we first assigned subjects to read some written text about COVID-19, and then presented subjects with a flier about COVID-19.

Written text. To introduce the written text portion of our treatments, we presented subjects with the following text: "Please read the following information about COVID-19, which the World Health Organization has recently classified as a pandemic." We then began by 
providing subjects with three paragraphs of basic information about coronavirus and the threat it poses. This portion of the written text was identical across treatments; see SI Section 6 for text. Next, we encouraged subjects to take the virus seriously and take preventative action. This portion of the written text varied across treatments. In the Personal treatment, it read:

For all of these reasons, coronavirus is a serious threat to you. It is recommended that you take this threat very seriously to prevent contracting COVID-19 and getting very ill or dying. Fortunately, there are steps you can take to keep yourself safe.

In the Public treatment, it read:

This means coronavirus is a serious threat to your community. It is recommended that you take this threat very seriously to prevent spreading COVID-19 and causing people in your community to get very ill or die. Fortunately, there are steps you can take to keep your community safe.

And in the Personal+Public treatment, it read:

This means coronavirus is a serious threat to you and your community. It is recommended that you take this threat very seriously to prevent contracting COVID-19 and getting very ill or dying, or spreading COVID-19 and causing people in your community to get very ill or die. Fortunately, there are steps you can take to keep yourself and your community safe.

Finally, the written text concluded by presenting a final paragraph encouraging subjects to engage in prevention behaviors. This portion of the text was constant across treatments; see SI Section 6 for text.

Thus, in the Personal treatment, we emphasized the threat to the subject, in the Public treatment, we emphasized the threat to the subject's community, and in the Personal+Public treatment, we emphasized the threat to the subject and their community. We note that the difference between the clause "for all these reasons" (Personal treatment) and "this means" (Public treatment and Public + Personal treatment) reflects an unintentional error; however, we believe that it is very unlikely to account for our results.

Furthermore, we note that our treatments varied slightly in length. However, we similarly believe that length differences are unlikely to account for our results. The length differences across treatments were quite minimal - and, as noted above, all treatments were accompanied by four paragraphs of text that was constant across treatments (and is reported in the SI). Thus, the percentage difference in word length across treatments is very small.

Fliers. After subjects finished reading this text, they were asked to carefully read a flier about COVID-19 (see Figure 1). To introduce the flier portion of our treatments, we presented subjects with the following text: "Thank you. Now, please carefully look at this flier, which provides further information about COVID-19." We then displayed a flier that varied across treatments, again by emphasizing threat to the subject, their community, or both. The images in the fliers were purchased from the stock photo provider istockphoto.com. 

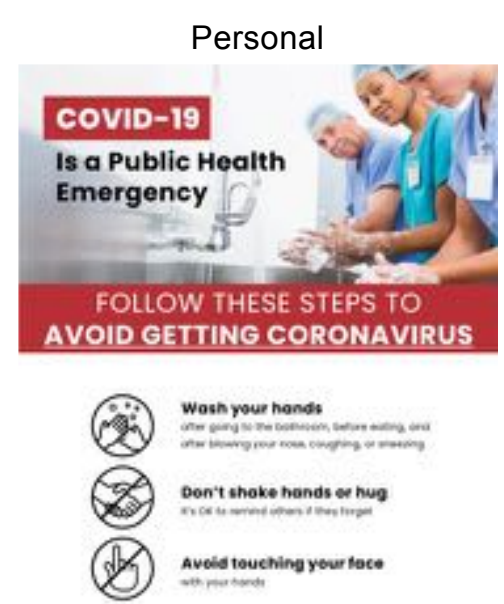

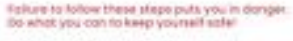

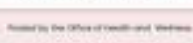

Public
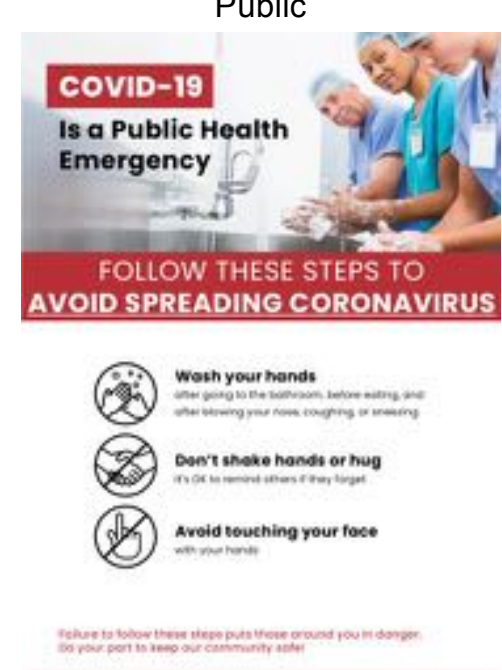
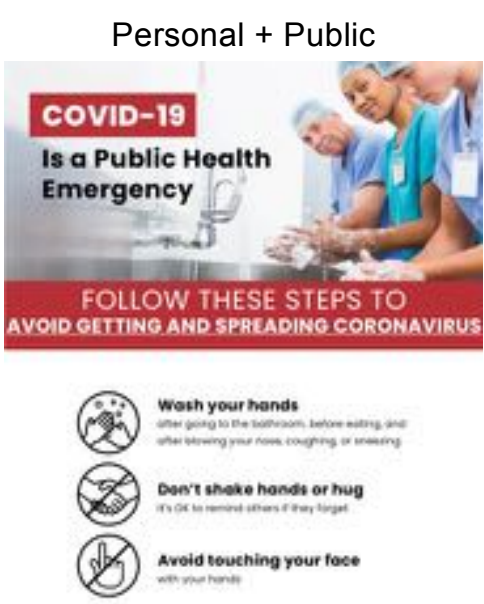

Figure 1. Fliers shown in each treatment. Photograph attribution: iStock.com/kali9.

\section{Measures}

After the treatments were presented to subjects in the treatment conditions, all subjects completed a series of outcome measures. We began by measuring (i) our two dependent variables and (ii) the perceived public and personal threat of coronavirus, manipulating betweensubjects which set of variables we measured first. Finally, we concluded by measuring a set of individual difference variables, described in SI Section 1.

Dependent variables. We collected two key dependent variables in a fixed order.

First, subjects reported their prevention intentions. To do so, they reported their intentions, on 0-100 sliding scales, to engage in a series of 11 prevention behaviors ("wash my hands at least 10 times a day", "wash my hands more often", "stop shaking other people's hands", "stop hugging people", "try my hardest to avoid touching my face", "stay home if I am feeling even a little bit sick", "try to stay home whenever possible, even if I am not sick", "cover my mouth when I cough and sneeze", "purchase food reserves and medication", and "stock up on cleaning supplies"). To create a composite measure of prevention intentions, we averaged intentions to engage in these 11 behaviors.

Next, subjects reported a more detailed set of social distancing intentions. While our above-described set of questions about prevention intentions included an overall social distancing item ("try to stay home whenever possible, even if I am not sick"), we also asked subjects a series of questions about specific activities they intended to avoid. In particular, we asked subjects to report their intentions, on 0-100 sliding scales, to avoid a set of 10 activities ("going to bars", "going to restaurants", "going to coffee shops", "going to the grocery store", "going to the gym", "going to work (somewhere outside of your home)", "using public transportation", "going to the airport and flying", "socializing in small gatherings", and "attending large events or gatherings"). Then, on a subsequent page, we asked subjects which of these activities they would engage in at least sometimes if coronavirus were not a concern. To create a composite measure of social distancing intentions, for each subject, we averaged intentions to avoid all activities that the subject indicated they would otherwise engage in.

Perceived personal and public threat of coronavirus. Additionally, we measured the perceived personal threat (to the subject) and public threat (to society) of coronavirus. We 
measured perceived public and personal threat on separate pages in random order. Each construct was measured via two parallel questions using on 0-100 sliding scales (which we averaged to form composite variables). The questions were (i) "To what extent are you afraid for yourself (your community) of contracting (contracting and spreading) coronavirus (i.e., COVID-19)? In other words, to what extent do you fear the personal repercussions for you (collective repercussions for society) that would follow from contracting (contracting and spreading) coronavirus?" and (ii) "How large of a personal (societal) threat do you think coronavirus (i.e., COVID-19) poses to you (your community)?", with italicized text corresponding to the personal threat measure and text in parentheses corresponding to the public threat measure. See SI Section 6 for more detail regarding these questions, including a description of a programming error that caused a minor difference in the way that we measured personal versus public threat.

We measured these constructs as potential mediating variables, reasoning that our treatments might function by influencing the perceived (personal or public) threat of coronavirus. Yet across all of our studies, we consistently found no significant treatment effects, or differences between treatments, on either threat variable (see SI Section 4.5 for details), suggesting that our treatments operated through other causal pathways (e.g., by increasing the perceived importance of prevention behaviors for avoiding harm to oneself or others). Thus, throughout this paper, instead of reporting analyses of our threat variables as potential mediators of our treatment effects, we instead report exploratory analyses of the associations between our threat variables and prevention intentions.

\section{Modifications for Study 2}

Study 2 was very similar to Study 1, with a few differences. First, we recruited a target of $n=1200$ subjects and assigned them to one of our three treatment conditions. Study 2 thus omitted the control condition that was included in Study 1 and had a larger target sample size per condition ( $n=400$ rather than $n=250)$. Second, we moved the measurement of all individual difference variables to the beginning of the study (i.e., before presenting our treatments), with the exception of performance on the Cognitive Reflection Task ${ }^{38}$ (which, like in Study 1, was measured last).

Third, we made a few modifications to the wording we used to measure the perceived public and personal threat of coronavirus. Specifically, for both our personal and public threat measures, we modified the first question to read "To what extent are you afraid of contracting (contracting and spreading) coronavirus (i.e. COVID-19) because of the consequences for you personally (for your community)?" Additionally, for our public threat measure, we modified the second question by replacing the phrase "societal threat" with "collective threat".

Finally, as our dependent variable, we only measured prevention intentions (and did not include our set of questions from Study 1 about more specific social distancing intentions). We made this decision because in Study 1, our measure of prevention intentions (which also included an overall social distancing item) produced stronger evidence for treatment effects and interesting differences between treatments. In Study 2, we thus chose to focus on replicating the observed effects on our measure of prevention intentions. For this reason, our analyses also focus more extensively on prevention intentions than our set of questions about more specific social distancing intentions. 


\section{Sample}

A total of $n=1,019$ subjects started Study 1 and $n=1,224$ subjects started Study 2. To form our final samples, when we collected duplicate responses from the same IP address or Mturk worker ID, we included only the chronologically first response. We also excluded responses from subjects who did not complete all key measures (defined as our dependent measures and potential mediators). This left us with $n=988$ subjects in Study 1 (mean age $=39$, $57 \%$ male) and $n=1188$ subjects (mean age $=37,60 \%$ male) in Study 2 .

\section{Analysis approach}

All of our analyses use linear regression. In all models aggregating data from both Studies 1 and 2, we include a study dummy. When computing Cohen's d values associated with regression results, we account for covariates (such as study dummies) by taking unstandardized regression coefficients (i.e., the difference between the covariate adjusted means for each group) as the numerator.

For analyses of our dependent variables, we report results (i) among all subjects, and, as a robustness check, (ii) among subjects for whom we measured our dependent variables before measuring our potential mediators. Although the latter analysis was not pre-registered, we include it to confirm the robustness of our results after having discovered an unexpected interaction between condition and the order in which we measured our dependent variables versus potential mediators. Specifically, in a regression predicting prevention intentions across both studies as a function of condition dummies, order, and their interactions, the interaction terms are jointly significant, $F(3,2167)=4.97, p=.002$.

We tested three treatments and measured two dependent variables, creating six possible comparisons (both when evaluating the effectiveness of each treatment relative to the control, and when comparing pairs of treatments). Thus, in addition to reporting $p$-values for these comparisons, we also report q-values, which indicate the probability of making at least one false discovery across these six comparisons when rejecting the null hypothesis for any result with an equal or smaller q-value. Specifically, we report calculated q-values (reported as $q_{c}$ ), derived from analytical calculations that conservatively assume that the tests for all six comparisons are independent from each other, and simulated q-values (reported as $q_{s}$ ), derived from simulations of our actual data that take into account the non-independence between some tests. See SI Section 2 for more details.

Finally, we note that in Study 1, we found some evidence that the Public treatment was relatively more effective for individuals reporting greater subjective health. Consequently, in our Study 2 pre-registration, we planned for our primary analyses to focus specifically on healthier individuals. However, evidence for an interaction between health and our Public treatment was weaker in Study 2 than in Study 1 (see SI Section 4.6 for details). Thus, we do not feel confident focusing on health in our primary analyses, and instead report analyses of all subjects. We note, however, that analyses of healthy individuals also support our key findings from Studies 1-2.

\section{Results}

\section{Comparisons of treatments to control}

We begin by comparing each of our treatment conditions to the control in Study 1 (which included a control condition). We thus conduct regressions predicting each of our two DVs, taking the control condition as the baseline and including dummies for the other three conditions. 
When investigating composite prevention intentions (across our 11-item scale, $\alpha=.89$ in each study), we find some robust evidence for treatment effects. We report the results of our analyses in Table 1 and plot prevention intentions across conditions in Figure 2. We find that subjects in all treatment conditions report directionally higher prevention intentions than subjects in the control condition, but only the Public condition shows a robust treatment effect that survives corrections for multiple comparisons.

$$
\text { All subjects }(n=988) \quad \text { Dependent variables first }(n=506)
$$

\begin{tabular}{|c|c|c|}
\hline Personal & $\begin{array}{c}\text { Control }=76.41[74.31,78.50] \\
\text { Personal }=79.19[76.98,81.40] \\
\mathbf{b}=\mathbf{2 . 7 8}, \mathbf{t}=\mathbf{1 . 8 9}, \mathbf{d}=\mathbf{0 . 1 7}, \\
\mathrm{p}=.059, \mathrm{q}_{\mathrm{c}}=.307, \mathrm{q}_{\mathrm{s}}=.245\end{array}$ & $\begin{array}{c}\text { Control }=74.49[71.21,77.77] \\
\text { Personal }=78.08[74.97,81.18] \\
\mathbf{b}=\mathbf{3 . 5 9}, \mathbf{t}=\mathbf{1 . 7 2}, \mathbf{d}=\mathbf{0 . 2 1} \\
\mathrm{p}=.086, \mathrm{q}_{\mathrm{c}}=.419, \mathrm{q}_{\mathrm{s}}=.345\end{array}$ \\
\hline Public & $\begin{array}{c}\text { Control }=76.41[74.31,78.50] \\
\text { Public }=81.88[80.11,83.64] \\
\mathbf{b}=\mathbf{5 . 4 7}, \mathbf{t}=\mathbf{3 . 7 0}, \mathbf{d}=\mathbf{0 . 3 3} \\
\mathrm{p}<.001, \mathrm{q}_{\mathrm{c}}=.001, \mathrm{q}_{\mathrm{s}}=.001\end{array}$ & $\begin{array}{c}\text { Control }=74.49[71.21,77.77] \\
\text { Public }=82.39[79.83,84.95] \\
\mathbf{b}=\mathbf{7 . 9 0}, \mathbf{t}=\mathbf{3 . 7 4}, \mathbf{d}=\mathbf{0 . 4 8} \\
\mathrm{p}<.001, \mathrm{q}_{\mathrm{c}}=.001, \mathrm{q}_{\mathrm{s}}=.001\end{array}$ \\
\hline $\begin{array}{c}\text { Personal + } \\
\text { Public }\end{array}$ & $\begin{array}{c}\text { Control }=76.41[74.31,78.50], \\
\text { Personal+Public }=79.76[77.67,81.85], \\
\mathbf{b}=\mathbf{3 . 3 5}, \mathbf{t}=\mathbf{2 . 2 6}, \mathbf{d}=\mathbf{0 . 2 0}, \\
\mathrm{p}=.024, \mathrm{q}_{\mathrm{c}}=.137, \mathrm{q}_{\mathrm{s}}=.110\end{array}$ & $\begin{array}{c}\text { Control }=74.49[71.21,77.77], \\
\text { Personal+Public }=82.22[79.69,84.75], \\
\mathbf{b}=\mathbf{7 . 7 3}, \mathbf{t}=\mathbf{3 . 6 4}, \mathbf{d}=\mathbf{0 . 4 7}, \\
\mathrm{p}<.001, \mathrm{q}_{\mathrm{c}}=.002, \mathrm{q}_{\mathrm{s}}=.001\end{array}$ \\
\hline
\end{tabular}

Table 1. Treatment effects on prevention intentions in Study 1. We compare each of our treatments to the control in Study 1. For each treatment, we report mean prevention intentions (with 95\% CIs) in the treatment and control conditions, and the treatment effect (both among all subjects, and subjects for whom we measured our dependent variables first).

A

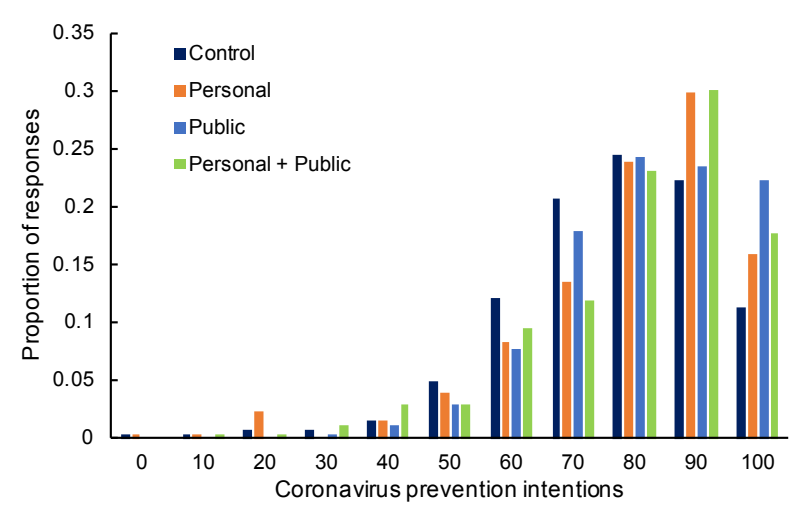

B

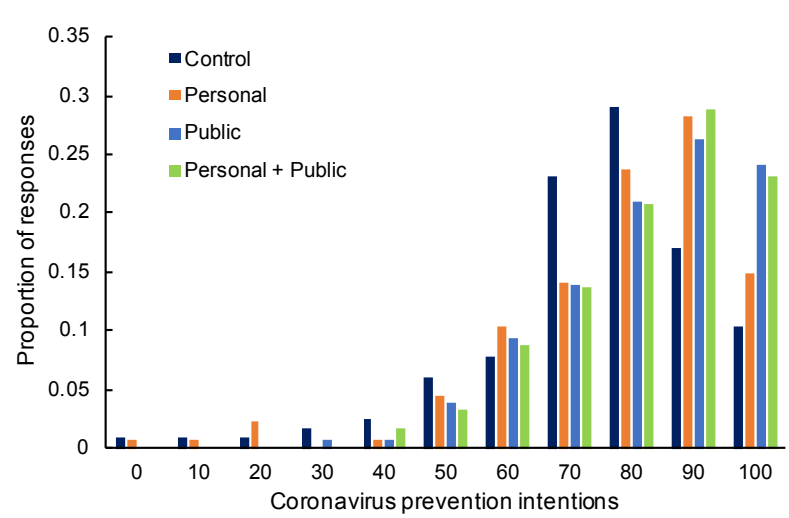

Figure 2. Prevention intentions by experimental condition in Study 1. Shown are frequencies of composite prevention intentions, rounded to zero or a multiple of ten, by experimental condition in Study 1, among all subjects (Panel $A, n=988$ ) and subjects for whom we measured our dependent variables first (Panel $B, n=506)$. 
When investigating composite social distancing intentions (across our 10-item scale investigating specific social distancing intentions collected in Study $1, \alpha=.91$ ), we do not find robust evidence for treatment effects (among all subjects, all ps $>.1$ and all qs $>.4$, although effects are somewhat stronger among subjects for whom we measured our dependent variables first); see SI Section 4.2 for more details.

\section{Comparisons between treatments}

Next, we turn to comparing the relative effectiveness of our different treatments. When investigating prevention intentions, which we measured in both studies, to maximize precision we pool data from both studies and report analyses from this combined dataset. We note, however, that we also plot results from each study individually, to illustrate the similarity across studies. We find that the Public treatment had the directionally largest effect on prevention intentions, and thus organize our results around comparing the Public treatment to the other two treatments. Throughout this paper, we conduct this comparison via regressions that take the Public treatment condition as the baseline and measure relative effectiveness of Public using dummies for the other two treatments.

The results, shown in Table 2 and Figure 3, provide robust evidence that the Public treatment was more effective than the Personal treatment. (In addition to surviving corrections for multiple comparisons, this conclusion also holds when accounting for the fact that our analyses of the pooled data across studies can be conceptualized as analyses of one study in which we "peeked" at the data after an initial collection, which can inflate type-I error rate. Following the approach of Sagarin et al., 2014, we calculate that under a "worst-case scenario" approach to data peeking, an adjusted alpha threshold of .028 is needed to maintain an actual type-I error rate of .05, and the observed $\mathrm{p}$ and $\mathrm{q}$ values fall below this threshold; see SI Section 3 for more details.) Furthermore, we find no significant difference between the effectiveness of the Public treatment and Personal+Public treatment. Thus, in Studies 1-2, we find evidence for the power of prosocial framing.

All subjects $(n=1930)$

\begin{tabular}{|c|c|c|}
\hline $\begin{array}{l}\text { Public vs. } \\
\text { Personal }\end{array}$ & $\begin{array}{c}\text { Public }=82.48[81.34,83.61] \\
\text { Personal }=79.93[78.66,81.19] \\
\mathbf{b}=\mathbf{2 . 5 5}, \mathbf{t}=\mathbf{2 . 9 0}, \mathbf{d}=\mathbf{0 . 1 6} \\
\mathrm{p}=.004, \mathrm{q}_{\mathrm{c}}=.022, \mathrm{q}_{\mathrm{s}}=.019\end{array}$ & $\begin{array}{r}\text { Public }=83.22[81.69,84 \\
\text { Personal }=79.42[77.65,8 \\
\mathbf{b}=\mathbf{3 . 8 0}, \mathbf{t}=\mathbf{3 . 2 5}, \mathbf{d}=\mathbf{0} . \\
\mathrm{p}=.001, \mathrm{q}_{\mathrm{c}}=.007, \mathrm{q}_{\mathrm{s}}=.\end{array}$ \\
\hline $\begin{array}{c}\text { Public vs. } \\
\text { Personal+Public }\end{array}$ & $\begin{array}{c}\text { Public }=82.48[81.34,83.61] \\
\text { Personal }+ \text { Public }=81.07[79.81,82.32] \\
\mathbf{b}=\mathbf{1 . 4 1}, \mathbf{t}=\mathbf{1 . 6 0}, \mathbf{d}=\mathbf{0 . 0 9} \\
\mathrm{p}=.109, \mathrm{q}_{\mathrm{c}}=.501, \mathrm{q}_{\mathrm{s}}=.408\end{array}$ & $\begin{array}{c}\text { Public }=83.22[81.69,84 \\
\text { Personal }+ \text { Public }=83.14[81.6 \\
\mathbf{b}=\mathbf{0 . 1 3}, \mathbf{t}=\mathbf{0 . 1 1}, \mathbf{d}=\mathbf{0} \\
\mathrm{p}=.913, \mathrm{q}_{\mathrm{c}}=1.000, \mathrm{q}_{\mathrm{s}}=\end{array}$ \\
\hline \multicolumn{3}{|c|}{$\begin{array}{l}\text { Table 2. Relative effects of the Public treatment on prevention intentions in Studies } 1 \text { and } 2 . \\
\text { We compare the Public treatment to each of our other treatments, across the treatment } \\
\text { conditions of Studies } 1 \text { and } 2 \text { combined. For each comparison, we report mean prevention } \\
\text { intentions (with 95\% CIs) by condition and the relative effect of the Public treatment (both } \\
\text { among all subjects, and subjects for whom we measured our dependent variables first). }\end{array}$} \\
\hline
\end{tabular}


A

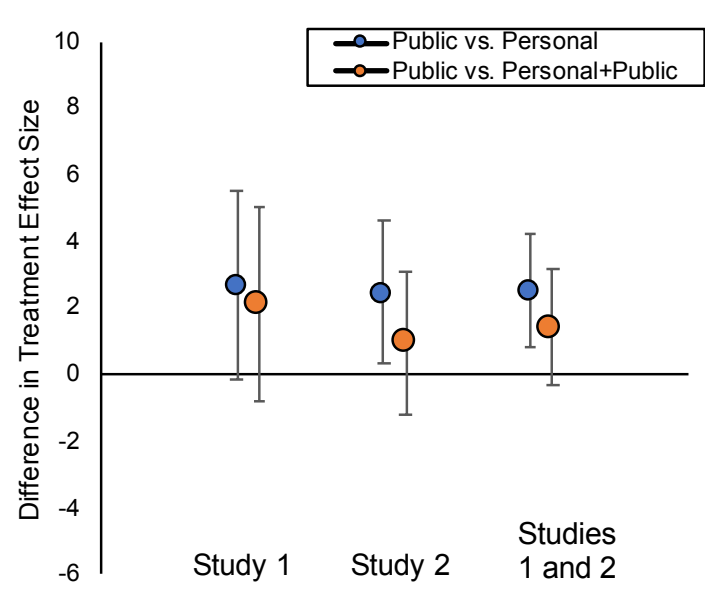

B

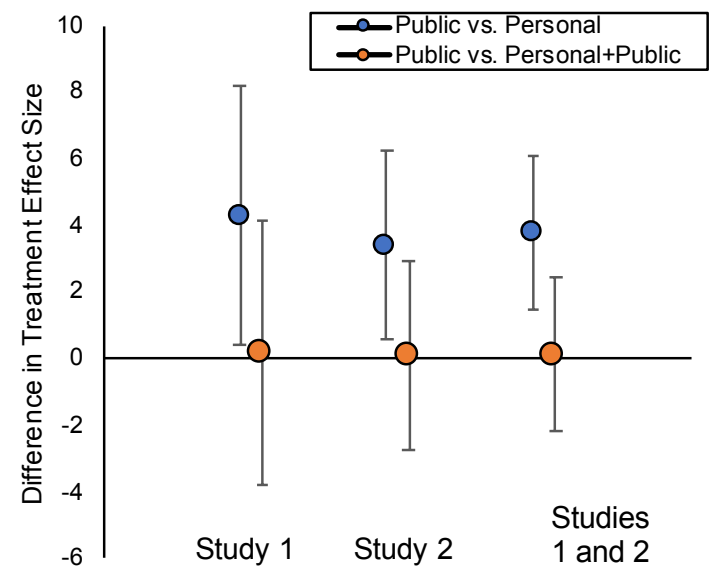

Figure 3. Relative effects of the Public treatment on prevention intentions in Studies 1 and 2. Shown are the relative effects of the Public treatment, as compared to the Personal treatment (blue dots) and Personal + Public treatment (orange dots). We plot results among all subjects (Panel A: Study $1 n=742$, Study $2 n=1188$, Studies 1 and 2 combined $n=1930$ ) and subjects for whom we measured our dependent variables first (Panel B: Study $1 n=389$, Study $2 n=$ 592, Studies 1 and 2 combined $n=981$ ).

When investigating composite social distancing intentions (from our 10-item scale investigating specific social distancing intentions, which we measured only in Study 1), we find no significant differences between any pairs of our treatments in any of our analyses (all ps $>.1$ and all qs $>.5$ ); see SI Section 4.2 for more details. However, the Public treatment was more effective than the Personal treatment at influencing the overall social distancing item included in our composite measure of prevention intentions ("try to stay home whenever possible, even if I am not sick"). In an aggregate analysis of Studies 1 and 2, we find a significant positive effect of a Public vs. Personal dummy on this item, both among all subjects, $b=3.70, t=2.83, d=.16, p$ $=.005$, and subjects for whom we measured our dependent variables first, $b=6.11, t=3.35, d=$ $.26, p=.001$. Thus, we find some evidence that the Public treatment may have been more effective than the Personal treatment at encouraging social distancing in Studies 1-2.

We also note that we find no clear evidence of heterogeneity in the effects of our treatments on prevention intentions either (i) across the 11 prevention behaviors we investigated (see SI Section 4.3), or (ii) across individuals based on the individual difference variables we collected (see SI Section 4.4).

Together, these results reveal that prosocial framing was more effective than selfinterested framing. Thus, we find evidence that it is valuable to encourage people to consider that prevention behaviors can, in additional to conferring personal benefits, also serve to benefit others.

\section{Perceived public and personal threat of coronavirus}

Next, we provide further support for the hypothesis that prosocial motivations play a central role in driving coronavirus prevention intentions by investigating the association between prevention intentions and the perceived public and personal threat of coronavirus. 
As illustrated in Table 3, we find that (i) both threat variables were positively associated with prevention intentions, and (ii) the association with prevention intentions is significantly stronger for perceived public than personal threat (although there was substantial covariance between threat variables, $r=.72$ in Study 1 and $r=.68$ in Study 2, ps $<.001$ ). These results hold in both Study 1 and Study 2 (despite the fact that we measured our threat variables slightly differently across studies), and are robust to including controls in our multiple regression models for age, gender, education, income, and political party affiliation.

Together, these results are consistent with the proposal that prosocial motivations play an important role in driving prevention intentions.

\begin{tabular}{|c|c|c|c|}
\hline & \multicolumn{3}{|c|}{ Study $1(n=988)$} \\
\hline & $\begin{array}{l}\text { Separate } \\
\text { models }\end{array}$ & $\begin{array}{l}\text { Multiple } \\
\text { regression }\end{array}$ & $\begin{array}{l}\text { Multiple } \\
\text { regression with } \\
\text { controls }\end{array}$ \\
\hline \multirow[t]{2}{*}{ Personal threat } & $0.412 * * *$ & 0.0730 & $0.0890 *$ \\
\hline & $(0.0290)$ & $(0.0393)$ & $(0.0398)$ \\
\hline \multirow[t]{2}{*}{ Public threat } & $0.522 * * *$ & $0.469 * * *$ & $0.451 * * *$ \\
\hline & $(0.0272)$ & $(0.0393)$ & $(0.0394)$ \\
\hline \multirow[t]{3}{*}{$\begin{array}{c}\text { Public vs. } \\
\text { Personal } \\
\text { comparison }\end{array}$} & $\begin{array}{c}\mathrm{t}(985)=5.40 \\
\mathrm{p}<.001\end{array}$ & $\begin{array}{c}\mathrm{F}(1,985)= \\
29.59 \\
\mathrm{p}<.001\end{array}$ & $\begin{array}{c}\mathrm{F}(1,980)= \\
24.05 \\
\mathrm{p}<.001\end{array}$ \\
\hline & \multicolumn{3}{|c|}{ Study $2(n=1188)$} \\
\hline & $\begin{array}{l}\text { Separate } \\
\text { models }\end{array}$ & $\begin{array}{l}\text { Multiple } \\
\text { regression }\end{array}$ & $\begin{array}{l}\text { Multiple } \\
\text { regression with } \\
\text { controls }\end{array}$ \\
\hline \multirow[t]{2}{*}{ Personal threat } & $0.401 * * *$ & $0.0652 *$ & $0.0843 * *$ \\
\hline & $(0.0266)$ & $(0.0332)$ & $(0.0325)$ \\
\hline \multirow[t]{2}{*}{ Public threat } & $0.540 * * *$ & $0.496 * * *$ & $0.498 * * *$ \\
\hline & $(0.0244)$ & $(0.0332)$ & $(0.0326)$ \\
\hline $\begin{array}{c}\text { Public vs. } \\
\text { Personal } \\
\text { comparison }\end{array}$ & $\begin{array}{c}t(1185)=7.02 \\
p<.001\end{array}$ & $\begin{array}{c}\mathrm{F}(1,1185)= \\
50.31, \mathrm{p}<.001\end{array}$ & $\begin{array}{c}F(1,1180)= \\
48.25, \mathrm{p}<.001\end{array}$ \\
\hline
\end{tabular}

Standard errors in parentheses

$* * * \mathrm{p}<0.001, * * \mathrm{p}<0.01, * \mathrm{p}<0.05$

Table 3. Associations between threat variables and prevention intentions in Studies 1 and 2. We report results from regressions predicting prevention intentions as a function of our threat variables. Shown are results from (i) a set of separate regression models for each threat variable (Column 1) and (ii) multiple regression models using both threat variables (Columns 2-3), for Study 1 (top rows) and Study 2 (bottom rows). We show results from multiple regression models both with and without controls for age, gender, education (coded here and in all analyses as a college degree dummy), income, and political party affiliation. All coefficients are standardized coefficients, with standard errors for each coefficient in parentheses. For each model, we also report results from a test comparing the public versus personal threat coefficient. 


\section{Study 3}

We now turn to describing of our second set of studies, which were conducted during in a later stage of the COVID-19 pandemic in the United States.

\section{Method}

\section{Overview}

On April 17 (Study 3a), April 22 (Study 3b), April 23 (Study 3c) and April 30 (Study 3d), 2020, we conducted four independent but almost identical "runs" of a study using convenience samples from Amazon Mechanical Turk. This set of studies (which we also refer to simply as "Study 3") was conceptually similar to Studies 1 and 2, and again investigated the effectiveness of our three treatments (Personal, Public, and Personal+Public). However, in Study 3 we updated our stimuli to account for the progression of the pandemic, including by emphasizing the longterm threat posed by coronavirus and measuring intentions to engage in prevention behaviors even after the conclusion of official stay-at-home orders (which were pervasive in the U.S. in April 2020). We also made some other meaningful design changes, described below.

Across all runs of Study 3, we assigned subjects to one of our three treatments. Instead of including a no-treatment control condition, we measured prevention intentions twice: once before subjects were exposed to their treatment (at "Time 1"), and once after subjects were exposed to their treatment (at "Time 2"). This design increased our power to detect treatment effects (conceptualized in Study 3 as differences between Time 1 and Time 2 prevention intentions), and also allowed us to investigate whether the relative effectiveness of our treatments varied as a function of Time 1 prevention intentions.

We conducted four runs of Study 3 because, in each of the first three runs (but not the fourth), we observed substantial differences in Time 1 (i.e., pre-treatment) prevention intentions across conditions. While we planned, in our pre-registrations, to control for Time 1 prevention intentions when predicting Time 2 prevention intentions, we were nonetheless concerned that the Time 1 imbalances might influence our results and thus chose to continue repeating the study. However, we ultimately found qualitatively identical results when either (i) analyzing only Study $3 \mathrm{~d}$ (in which there was no substantial Time 1 imbalance) or (ii) conducting an aggregate analysis of Studies 3a-d; for completeness, we present results from both analysis approaches.

In Studies 3a-c, we recruited $n=750$ subjects ( 250 per condition). In Study $3 d$, (i) we recruited a larger sample of $n=1800$ subjects ( 600 per condition), in order to reduce the probability of again observing imbalanced Time 1 prevention intentions across conditions, and correspondingly (ii) shortened the study by measuring fewer individual difference variables.

\section{Treatments}

Like in Studies 1-2, our Study 3 treatments again consisted of a written text and a flier. However, as noted above, we updated our stimuli. To introduce the written text portion of our treatments, we presented subjects with the following text: "Please read the following information about COVID-19." In all treatments, the written text then began with two paragraphs describing the ongoing threat posed by coronavirus and emphasizing the possibility of new outbreaks; see SI Section 6 for text. The written text then preceded to a new page, which varied across treatments. The Personal treatment read: 
For these reasons, coronavirus is likely to remain a serious threat to you for the foreseeable future. It is important that you continue to take this threat very seriously to prevent contracting COVID-19 and getting very ill or dying. It is recommended that you continue to take the necessary steps to keep yourself safe from infection now, and from new outbreaks in the future.

The Public treatment read:

For these reasons, coronavirus is likely to remain a serious threat to your community for the foreseeable future. It is important that you continue to take this threat very seriously to prevent spreading COVID-19 and causing people in your community to get very ill or die. It is recommended that you continue to take the necessary steps to keep your community safe from infection now, and from new outbreaks in the future.

And the Personal+Public treatment read:

For these reasons, coronavirus is likely to remain a serious threat to you and your community for the foreseeable future. It is important that you continue to take this threat very seriously to prevent contracting COVID-19 and getting very ill or dying, or spreading COVID-19 and causing people in your community to get very ill or die. It is recommended that you continue to take the necessary steps to keep yourself and your community safe from infection now, and from new outbreaks in the future.

Finally, in all treatments, the written text concluded by presenting two paragraphs encouraging subjects to continue engaging in prevention behaviors, even after official stay at home orders end, and noting the potential importance of contract tracing and testing efforts; see SI Section 6 for text. We note that, as was true for Studies 1-2, our treatments again varied slightly in length, but the length differences were again quite minimal (especially in the context of the text that was constant across treatments).

We also updated our fliers, illustrated in Figure 4. To introduce the flier portion of our treatments, in Studies 3a-c, we presented subjects with the following text: "Thank you. Now, please carefully look at this flier, which provides further guidelines for behavior over the next few weeks, while stay at home orders and other official social distancing measures remain in place". By the time we ran Study 3d, some states were beginning to lift such measures, so we instead used the text "Thank you. Now, please carefully look at this flier, which provides guidelines for behavior relevant to COVID-19." The images in the fliers were again purchased from the stock photo provider istockphoto.com. 

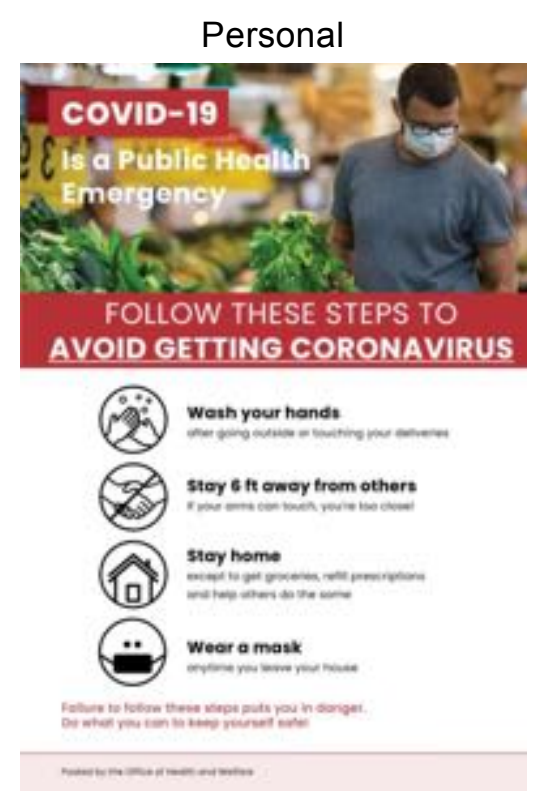
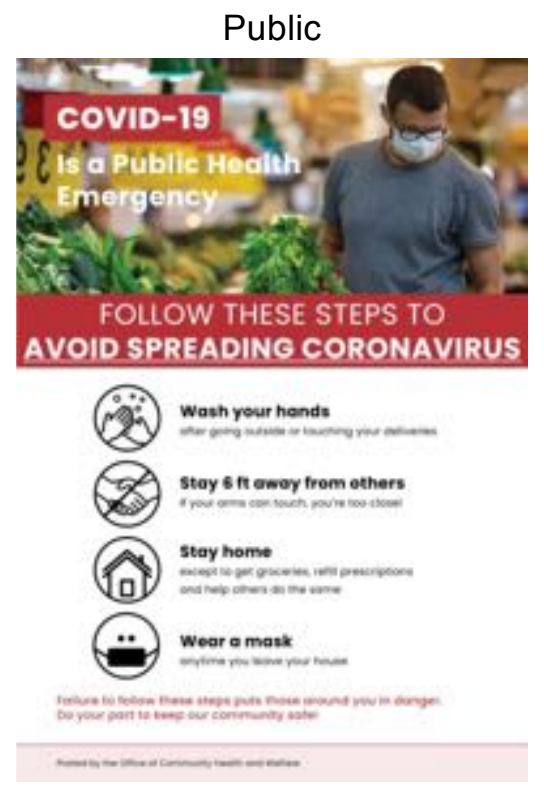
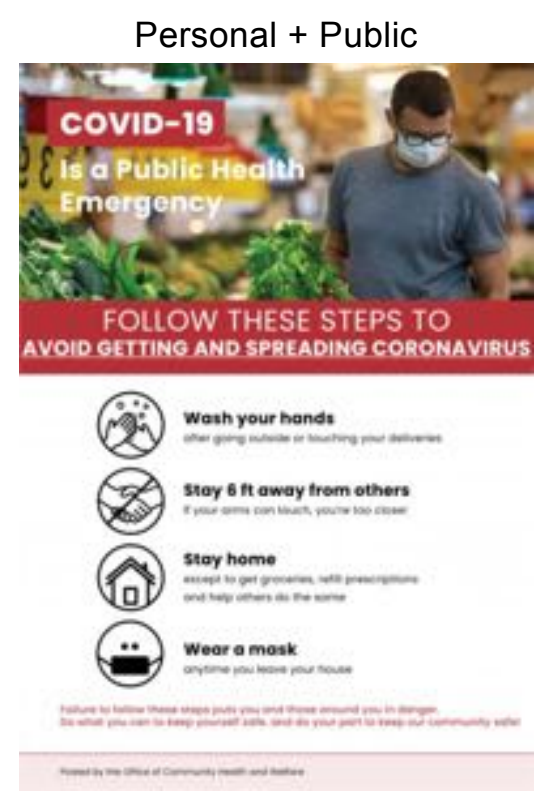

Figure 4. Fliers shown in each treatment across Studies 3a-d. Photograph attribution: iStock.com/Space_Cat.

\section{Measures}

In Study 3, we began by measuring prevention intentions, and then exposed subjects to a randomly assigned treatment. Next, we re-measured prevention intentions, presented subjects with an attention check (in Studies 3a-c), measured our threat variables, and concluded by measuring a set of individual difference variables (including a second attention check in Studies 3a-c) described in SI Section 1.

Prevention intentions. As in Studies 1-2, in Study 3 we measured prevention intentions via 0-100 sliding scales. To limit anchoring effects, we modified the sliders so that subjects were not shown a numeric value that corresponded to their responses. Additionally, we updated our measure of prevention intentions by (i) asking subjects specifically about their intentions "even after official stay-at-home orders end", and (ii) measuring intentions to engage in a different set of 10 prevention behaviors.

Specifically, Study 3 measured intentions to "wash my hands as much as possible for the foreseeable future", "try my hardest to avoid touching my face for the foreseeable future", "limit my physical interaction with others when possible for the foreseeable future", "refrain from visiting anyone who is sick at the hospital, even if they are a close family member and even if they are dying", "if relevant, allow the government to track my health data, movements, and/or the people I interact with", "if relevant, allow the government to regularly test me for COVID19", "wear a mask when I leave the house for the foreseeable future," "completely avoid any unnecessary physical contact with others (e.g., hugging or handshakes) for the foreseeable future", "avoid crowded indoor or outdoor spaces for the foreseeable future", and "even if I think I have contracted and recovered from COVID-19, remain vigilant unless I have a confirmed positive test (either for the virus during infection, or antibodies after infection)".

Perceived public and personal threat of coronavirus. We measured the perceived public and personal threat of coronavirus as in Study 2 (and correcting the programming error present in Studies 1-2). 


\section{Sample}

A total of $n=4,174$ subjects started Studies $3 \mathrm{a}-\mathrm{d}$ and $n=1,846$ subjects started Study $3 \mathrm{~d}$. We formed our final Study 3 samples as in Studies 1-2, leaving us with $n=3985$ subjects across Studies 3a-d (mean age $=38,55 \%$ male) and $n=1773$ subjects in Study $3 \mathrm{~d}$ (mean age $=39$, $48 \%$ male).

\section{Analysis approach}

Because of the imbalance in Time 1 prevention intentions across treatments in Studies 3a-c, when investigating effects of our treatments on dependent measures, we report results both (i) across Studies 3a-d (and include study dummies in our models), and (ii) in Study 3d only.

As in Studies 1-2, our Study 3 analyses correct for multiple comparisons. Study 3 tested three treatments and measured one dependent variable; thus, we report q-values that indicate the probability of false discovery across three comparisons. However, because the results of Study 3 are clearly definitive (specifically, in supporting the conclusions that all treatments were effective, and there were no meaningful differences in effectiveness between treatments), we simply report (more conservative) calculated q-values $\left(q_{c}\right)$ and do not generate simulated qvalues that account for the non-independent between some tests.

In our Study 3 pre-registrations, we planned to analyze our data in long format (with one observation per prevention intention item per subject). Yet for consistency with our approach from Studies 1-2, we instead primarily analyze data in wide format, and compute composite prevention intentions across our 10 items (across Studies 3a-d, Time $1 \alpha=.87$ and Time $2 \alpha=$ .89). When investigating whether the relative effectiveness of treatments is influenced by Time 1 prevention intentions, however, we do analyze the data in long format (allowing us to address this research question more precisely). We note that our conclusions from all analyses are qualitatively unchanged when analyzing in wide versus long format.

\section{Results}

\section{Effectiveness of treatments}

We begin by investigating whether each of our treatments was effective at increasing prevention intentions. To this end, we shape our data to have two observations per subject (for composite prevention intentions at Times 1 and 2, respectively). Then, for each treatment, we predict prevention intentions as a function of time, with robust standard errors clustered on subject. As illustrated by Table 4, we find strong evidence that all three treatments were effective at increasing prevention intentions. 


\begin{tabular}{|c|c|c|}
\hline & Studies 3a-d $(n=3985)$ & Study 3d $(n=1773)$ \\
\hline Personal & 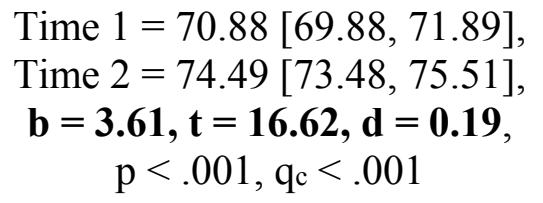 & $\begin{array}{c}\text { Time } 1=70.41[68.87,71.96], \\
\text { Time } 2=74.01[72.41,75.62], \\
\mathbf{b}=\mathbf{3 . 6 0}, \mathbf{t}=\mathbf{1 2 . 3 6}, \mathbf{d}=\mathbf{0 . 1 8}, \\
\mathrm{p}<.001, \mathrm{q}_{\mathrm{c}}<.001\end{array}$ \\
\hline Public & $\begin{array}{c}\text { Time } 1=73.46[72.50,74.42] \\
\text { Time } 2=76.73[75.77,77.68] \\
\mathbf{b}=\mathbf{3 . 2 7}, \mathbf{t}=\mathbf{1 4 . 9 9}, \mathbf{d}=\mathbf{0 . 1 8} \\
\mathrm{p}<.001, \mathrm{q}_{\mathrm{c}}<.001\end{array}$ & $\begin{array}{c}\text { Time } 1=71.23[69.73,72.73], \\
\text { Time } 2=75.04[73.50,76.58], \\
\mathbf{b}=\mathbf{3 . 8 1}, \mathbf{t}=\mathbf{1 2 . 0 2}, \mathbf{d}=\mathbf{0 . 2 0}, \\
\mathrm{p}<.001, \mathrm{q}_{\mathrm{c}}<.001\end{array}$ \\
\hline $\begin{array}{l}\text { Personal } \\
+ \text { Public }\end{array}$ & 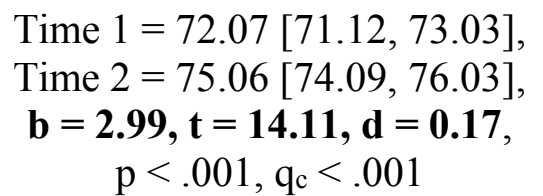 & $\begin{array}{c}\text { Time } 1=70.84[69.38,72.30], \\
\text { Time } 2=74.37[72.86,75.89], \\
\mathbf{b}=\mathbf{3 . 5 3}, \mathbf{t}=\mathbf{1 0 . 6 6}, \mathbf{d}=\mathbf{0 . 1 9}, \\
\mathrm{p}<.001, \mathrm{q}_{\mathrm{c}}<.001\end{array}$ \\
\hline
\end{tabular}

Table 4. Effectiveness of treatments in Study 3. For each treatment, we report mean Time 1 (i.e., pre-treatment) and Time 2 (i.e., post-treatment) prevention intentions, and the change over time (across Studies 3a-d, and in Study $3 d$ only).

\section{Comparisons between treatments}

Next, we turn to comparing the relative effectiveness of our different treatments. To this end, we shape our data to have one observation per subject (with two variables for composite prevention intentions at Times 1 and 2, respectively). Then, we predict Time 2 prevention intentions as a function of treatment dummies, controlling for Time 1 prevention intentions. As illustrated by Table 5, we find that our three treatments were similarly effective.

\begin{tabular}{ccc} 
& Studies 3a-d $(\boldsymbol{n}=\mathbf{3 9 8 5})$ & Study 3d $(\boldsymbol{n}=\mathbf{1 7 7 3})$ \\
\hline Public vs. & $\mathbf{b}=\mathbf{- \mathbf { 0 . 1 2 }}, \mathbf{t}=\mathbf{- 0 . 4 2}, \mathbf{d}=-$ & $\mathbf{b}=\mathbf{0 . 2 5}, \mathbf{t}=\mathbf{0 . 5 7}, \mathbf{d}=\mathbf{0 . 0 1}$, \\
Personal & $\mathbf{0 . 0 1}$, & $\mathrm{p}=.567, \mathrm{q}_{\mathrm{c}}=.919$ \\
\hline Public vs. & $\mathbf{b}=\mathbf{0 . 4 0}, \mathbf{t}=\mathbf{1 . 3 3}, \mathbf{d}=\mathbf{0 . 0 2}$, & $\mathbf{b}=\mathbf{0 . 3 0}, \mathbf{t}=\mathbf{0 . 6 8}, \mathbf{d}=\mathbf{0 . 0 2}$, \\
Personal+Public & $\mathrm{p}=.182, \mathrm{q}_{\mathrm{c}}=.453$ & $\mathrm{p}=.498, \mathrm{q}_{\mathrm{c}}=.874$ \\
\hline Pesonal vs. & $\mathbf{b}=\mathbf{0 . 5 2}, \mathbf{t}=\mathbf{1 . 7 5}, \mathbf{d}=\mathbf{0 . 0 3}$, & $\mathbf{b}=\mathbf{0 . 0 5}, \mathbf{t}=\mathbf{0 . 1 0}, \mathbf{d}=\mathbf{0 . 0 0}$, \\
Personal+Public & $\mathrm{p}=.080, \mathrm{q}_{\mathrm{c}}=.220$ & $\mathrm{p}=.918, \mathrm{q}_{\mathrm{c}}=.999$
\end{tabular}

Table 5. Comparisons between treatments in Study 3. For each pairwise comparison, we report the relative effect of the treatment listed first on Time 2 prevention intentions, when controlling for Time 1 prevention intentions (across Studies 3a-d, and in Study 3d only).

We also find no robust evidence that the relative effectiveness of our treatments varies as a function of Time 1 prevention intentions; see SI Section 4.7 for more detail.

\section{Perceived public and personal threat of coronavirus}

Next, we turn to investigating the association between prevention intentions and the perceived public and personal threat of coronavirus. Given that our threat variables did not 
mediate our treatment effects in Studies 1 and 2, our Study 3 pre-registrations simply planned to investigate absolute values of perceived threat (for the purpose of comparison to Studies 1 and 2). (See SI Section 4.5 for this comparison, as well as evidence that, as in Studies 1-2, in Study 3 we again found no differences between our treatments on either threat variable.)

However, we again investigate the association between prevention intentions and our threat variables in order to shed further light on the extent to which prevention intentions are motivated by prosociality versus self-interest. These analyses are not pre-registered, but nonetheless serve as a confirmatory test of whether our association results from Studies 1-2 replicate in Study 3.

As illustrated in Table 6, across Studies 3a-d we find that (i) both threat variables were associated with composite prevention intentions, and (ii) the association is significantly stronger for perceived public than personal threat (although there was substantial covariance between threat variables, $r=.73$ ). These results hold when predicting composite prevention intentions at either Time 1 or Time 2, and are robust to including controls for age, gender, education, income, political party affiliation, and race (note that we control for race in our analyses of Study 3, but not Studies 1-2, because race was only measured in Study 3 ).

Together, these results are consistent with the proposal that prosocial motivations play an important role in driving prevention intentions.

\begin{tabular}{|c|c|c|c|}
\hline & \multicolumn{3}{|c|}{ Time 1 prevention intentions } \\
\hline & $\begin{array}{l}\text { Separate } \\
\text { models }\end{array}$ & $\begin{array}{l}\text { Multiple } \\
\text { regression }\end{array}$ & $\begin{array}{c}\text { Multiple } \\
\text { regression } \\
\text { with controls }\end{array}$ \\
\hline \multirow[t]{2}{*}{ Personal threat } & $0.505 * * *$ & $0.185 * * *$ & $0.170 * * *$ \\
\hline & $(0.0138)$ & $(0.0190)$ & $(0.0191)$ \\
\hline \multirow[t]{2}{*}{ Public threat } & $0.570 * * *$ & $0.435 * * *$ & $0.437 * * *$ \\
\hline & $(0.0131)$ & $(0.0190)$ & $(0.0193)$ \\
\hline \multirow[t]{3}{*}{$\begin{array}{c}\text { Public vs. } \\
\text { Personal } \\
\text { comparison }\end{array}$} & $\begin{array}{c}\mathrm{t}(3982)=6.94 \\
\mathrm{p}<.001\end{array}$ & $\begin{array}{c}F(1,3979)= \\
49.65, p<.001\end{array}$ & $\begin{array}{c}F(1,3967)= \\
55.73, p<.001\end{array}$ \\
\hline & \multicolumn{3}{|c|}{ Time 2 prevention intentions } \\
\hline & $\begin{array}{l}\text { Separate } \\
\text { models }\end{array}$ & $\begin{array}{l}\text { Multiple } \\
\text { regression }\end{array}$ & $\begin{array}{c}\text { Multiple } \\
\text { regression } \\
\text { with controls }\end{array}$ \\
\hline \multirow[t]{2}{*}{ Personal threat } & $0.505 * * *$ & $0.160 * * *$ & $0.145 * * *$ \\
\hline & $(0.0138)$ & $(0.0189)$ & $(0.0188)$ \\
\hline \multirow[t]{2}{*}{ Public threat } & $0.587 * * *$ & $0.469 * * *$ & $0.471 * * *$ \\
\hline & $(0.0129)$ & $(0.0188)$ & $(0.0190)$ \\
\hline $\begin{array}{c}\text { Public vs. } \\
\text { Personal } \\
\text { comparison }\end{array}$ & $\begin{array}{c}\mathrm{t}(3982)=8.70 \\
\mathrm{p}<.001\end{array}$ & $\begin{array}{c}\mathrm{F}(1,3979)= \\
77.43, \mathrm{p}<.001\end{array}$ & $\begin{array}{c}F(1,3967)= \\
85.30, p<.001\end{array}$ \\
\hline
\end{tabular}

Standard errors in parentheses

$* * * \mathrm{p}<0.001,{ }^{* *} \mathrm{p}<0.01,{ }^{*} \mathrm{p}<0.05$

Table 6. Associations between threat variables and prevention intentions in Study 3. We report results from regressions predicting prevention intentions as a function of our threat variables, across Studies 3a-d. Shown are results from (i) a set of separate regression models for each 
threat variable (Column 1) and (ii) multiple regression models using both threat variables (Columns 2-3), when predicting prevention intentions at Time 1 (top rows) and Time 2 (bottom rows). We show results from multiple regression models both with and without controls for age, gender, education, race, income, and political party affiliation. All coefficients are standardized coefficients, with standard errors for each coefficient in parentheses. For each model, we also report results from a test comparing the public versus personal threat coefficient.

\section{Study 4}

In Study 4, we investigate the generalizability of the associations between our threat variables and prevention intentions to a more representative sample and different operationalizations of perceived public and personal threat.

\section{Method}

To do so, we conduct a novel reanalysis of data from Pennycook, McPhetres, Bago \& Rand (2020), who used Prolific to recruit a sample of $n=748$ American subjects on March $24^{\text {th }}$, 2020 that matched the national distribution on age, gender, ethnicity, and geographic region. Subjects completed the same prevention intentions measure from Studies 1 and 2. As in our previous analyses, we averaged responses to these items to create a composite intentions score. Additionally, subjects used 7-point Likert scales to indicate their agreement with various statements related to the threat posed by COVID-19. Our main text analysis focuses on the items that we see as mapping most closely onto the constructs of public threat ("The coronavirus poses a major threat to the public") and personal threat ("Because of my age and/or pre-existing conditions, I am likely to have serious symptoms if I were to contract the coronavirus" and "Because of my age and/or pre-existing conditions, I am likely to need hospitalization if I were to contract the coronavirus"; averaged to create a personal threat index). In SI Section 4.10 we demonstrate that the results are robust to alternative choices about which items to use (including using all items collected).

We note that the Study 4 correlation between perceived public threat and our perceived personal threat index $(r=.24)$ was substantially lower than in Studies 1-3 (although still highly statistically significant, $\mathrm{p}<0.001$ ). This reduces any potential concerns of multiple-collinearity that may have arisen in the previous analyses.

For full methodological details and materials, see Pennycook et al. (2020) and https://osf.io/3a497/.

\section{Results}

As illustrated in Table 7, and consistent with our results from Studies 1-3, we again find that (i) both personal and public threat are associated with prevention intentions, and (ii) the association is significantly stronger for perceived public than personal threat. Our results are also robust to including controls in the multiple regression model for age, gender, education, race, income, and political party affiliation. Thus, we find that the correlational results observed in Studies 1-3 generalize to a more representative sample, and different measures of threat. 


\begin{tabular}{cccc}
\hline & Separate models & $\begin{array}{c}\text { Multiple } \\
\text { regression }\end{array}$ & $\begin{array}{c}\text { Multiple } \\
\text { regression with } \\
\text { controls }\end{array}$ \\
\hline Personal threat & $\mathbf{0 . 2 8 2 * * *}$ & $\mathbf{0 . 1 9 1 * * *}$ & $\mathbf{0 . 1 7 9 * * *}$ \\
Public threat & $(0.0351)$ & $(0.0336)$ & $(0.0397)$ \\
Public vs. & $\mathbf{0 . 4 1 8 * * *}$ & $\mathbf{0 . 3 7 1} * * *$ & $\mathbf{0 . 3 5 5 * * *}$ \\
$\begin{array}{c}\text { Personal } \\
\text { comparison }\end{array}$ & $(0.0333)$ & $(0.0336)$ & $(0.0354)$ \\
\hline
\end{tabular}

Standard errors in parentheses

$* * * \mathrm{p}<0.001,{ }^{* *} \mathrm{p}<0.01, * \mathrm{p}<0.05$

Table 7. Associations between threat variables and prevention intentions in Study 4. We report results from regressions predicting prevention intentions as a function of the composition thread variables collected in Study 4. Shown are results from (i) a set of separate regression models for each threat variable (Column 1) and (ii) multiple regression models using both threat variables (Columns 2-3). We show results from multiple regression models both with and without controls for age, gender, education, race, income, and political party affiliation. All coefficients are standardized coefficients, with standard errors for each coefficient in parentheses. For each model, we also report results from a test comparing the public versus personal threat coefficient.

\section{Study 5}

In Study 5, we provide a final source of support for the proposal that prosocial motivates contribute to prevention intentions. Specifically, we investigate the association between prevention intentions across Studies 1-3 and prosocial behavior in incentivized economic game experiments conducted years prior to the pandemic.

\section{Method}

To this end, we draw on an external dataset of MTurk studies conducted by members of our research group. All of these studies included an economic "Dictator Game" (DG), in which subjects received an endowment of money (which varied across studies) and were asked to allocate it between themselves and another anonymous MTurk worker (in increments that varied across studies). Subjects faced a financial incentive to selfishly keep the money for themselves: in all studies, subjects' DG decisions influenced their bonus payment. Thus, we take the percentage of the starting endowment that subjects chose to allocate to the other Mturk worker as a measure of prosocial behavior.

This dataset compiled 44 different studies ${ }^{40-42}$, conducted between 2012 and 2018, and included 37,622 unique decisions made by 23,170 unique subjects (as indexed by Mturk Worker IDs). For subjects who made more than one DG decision, we computed the average across their decisions. 
We then investigated the association between prevention intentions in Studies 1-3 and previous DG decisions. To this end, we merged our data from Studies 1-3 with our DG dataset (using Mturk Worker IDs), resulting in DG data for a total of $n=1,522$ subjects across Studies 1-3. To conduct an aggregate analysis of Studies 1-3, we used our composite measure of prevention intentions from Studies 1-2 and our composite measure of Time 1 prevention intentions from Studies 3a-d.

\section{Results}

We found a small but significant positive association between prevention intentions and DG decisions $(B=.08, t=3.42, p=.001)$ that is robust to controlling for age, gender, education, race, income, and political party affiliation $(B=.06, t=2.54, p=.011)$. We note that we also find similar results when using Time 2 prevention intentions from Studies 3a-d (without controls $B=.09, t=3.75, p<.001$, with controls $B=.07, t=2.69, p=.007)$. We also note that in our models with controls, because race was only measured in Study 3, we recoded our dummies for each racial category to include a third value for missing data.

Thus, we find evidence that prosociality, as measured by incentive-compatible behavior in the Dictator Game (conducted years prior to the coronavirus pandemic), is positively associated with coronavirus prevention intentions. This result provides further support for the proposal that prosocial motives contribute to prevention intentions.

\section{Study 6}

Finally, in Study 6 we present a field experiment testing messaging aimed at promoting the installation of a contact tracing app. Contact tracing apps use Bluetooth on users' mobile phones to identify which users have been in close proximity to each other. They then can notify a user if they have been in close proximity with somebody who has tested positive for COVID-19. This allows the notified individual to get tested when they otherwise might not have, and, if they test positive, to seek care earlier than they otherwise might have, and to take measures to avoid infecting others. Using such apps thus has benefits both for oneself and for others, mirroring other COVID-19 prevention behaviors. In December 2020, the time that we conducted our field experiment, contact tracing apps were available in roughly half of U.S. states and were touted by experts as one of the most effective tools in combating the spread of COVID-19 before vaccines became more widely available.

\section{Method}

Our field experiment was performed in collaboration with COVID Act Now (CAN). CAN is one of the three largest COVID-19 websites, as measured by daily visits. CAN provides key statistics about the pandemic at a national, state, and county level, and also sends a daily newsletter with COVID-19 news. This newsletter was the setting for our experiment.

Our experiment was performed on three consecutive days from Monday December 21 to Wednesday December 23, 2020. Our sample was the entirety of CAN's newsletter subscribers, which numbered 152,556 at the time. Although our goal was for users to be perfectly randomized to condition, our field partner implemented the following procedure. 
The sample was divided into three treatment groups. Assignment to groups was performed based on last name: subscribers with last names that began with the letters A through I were assigned to group one $(n=59,352)$, subscribers with last names that began with the letters $\mathrm{J}$ through Q were assigned to group two $(n=56,978)$, and the remaining subscribers were assigned to group three $(n=36,226)$.

On all three days of the experiment, all subscribers were emailed a newsletter. The email subject lines and content of these newsletters was different on each of these three days. To implement our treatments, on each day, just one of the treatment groups received a short "header" in the body of their email, at the beginning of their newsletter. This header briefly explained what contact tracing apps are and encouraged the reader to download such an app. It also provided a link where readers could see if their state had a contact tracing app available, and, if so, could download the app.

There were three versions of the header. The version sent on Monday December 21 was sent to subscribers in group one, and emphasized the personal benefits of downloading a contact tracing app (Personal treatment): "It lets you know if you may have COVID, which helps you obtain treatment more quickly." The version sent on Tuesday December 22 was sent to subscribers in group two, and emphasized the benefits that downloading an app has for others (Public treatment): "It lets you know if you may have COVID, which prevents you from spreading COVID to more people". Finally, the version sent on Wednesday December 23 was sent to subscribers in group three, and emphasized both benefits (Personal+Public treatment): "It lets you know if you may have COVID, which helps you obtain treatment more quickly and prevents you from spreading COVID to more people".

For complete copies of the newsletters sent each day, see SI Section 6.5.

\section{Results}

Our key outcome measure is the click-through rate: the fraction of people who clicked the link to the contract tracing app, among those who opened the email (and thus saw the header prompting them to download the contact tracing app). As described above, our three treatments displayed the relevant header on three different dates, and in the context of three different email subject lines. Thus, overall email open rates varied slightly across days (reflecting effects either of date or email subject line); in particular, $40.28 \%(n=23,908)$ opened the email on $12 / 21$, $41.50 \%(n=23,644)$ opened the email on $12 / 22$, and $37.98 \%(n=13,760)$ opened the email on $12 / 23$. Thus, we cannot simply compare the total number of link clicks on each of these days (as differences could reflect unequal rates of opening the email, rather than the influence of our messaging in the headers). We therefore instead ask: among those who opened their email and saw their header, what fraction chose to click the link?

We find that the click-through rate in the Personal treatment $(6.27 \%)$ did not differ significantly from the click-through rate in the Public treatment (6.64\%); two-sample test of proportions: $\mathrm{z}=-1.64, p=.100$. However, the click-through rate in the Personal + Public treatment $(7.33 \%)$ was significantly higher than the rate observed in either the Personal treatment $(\mathrm{z}=3.98, p<.001)$ or the Public treatment $(\mathrm{z}=2.55, p=.011)$.

By examining actual link-clicking behavior in the field (rather than self-reported behavioral intentions in a survey experiment), Study 6 provides externally valid evidence for the power of prosocial framing. A header that highlighted the personal and public benefits of 
contract tracing resulted in higher click-through rates than headers highlighting either only the personal benefits or only the public benefits.

It is important to note that because of the imperfect randomization, this evidence is only suggestive. First, recall that assignment to treatment was not random, but rather based on subscriber last name. Thus, it is possible that the observed differences between treatments reflect that subscribers with different last names behave differently. Second, recall that our three treatments were presented on different dates and in the context of different email subject lines, resulting in different open rates across days. If different types of subscribers opened the email on different days, it is possible that the observed differences between treatments reflect that some types were more inclined to click the link than others. Finally, recall that our three treatments were presented in the context of different newsletter content (that was displayed below the header with our messaging). Thus, it is possible that the observed differences between treatments reflect that these differences in newsletter content influenced click-through rates. Thus, while Study 6 provides suggestive evidence for the power of combined personal and public framing in a field context, future work should seek to conceptually replicate these results using a more appropriate randomization procedure.

\section{Discussion}

Coronavirus prevention efforts can reasonably be conceptualized as either self-interested or prosocial. We have investigated which framing is more effective - and motivation is stronger-for fostering intentions to engage in prevention behaviors.

First, we investigated the relative efficacy of self-interested (“don't get it") versus prosocial ("don't spread it") messaging. In a set of studies conducted earlier in the pandemic (March 14-16, 2020, at which time there were fewer than 2000 U.S. cases), prosocial framing was more effective than self-interested framing. Furthermore, combining self-interested and prosocial framing was no more effective than pure prosocial framing. In a set of studies conducted slightly later in the pandemic (April 17-30, 2020, at which time hundreds of thousands of Americans had been infected), all three framing strategies were similarly effective. Finally, in a field experiment conducted more than half a year later (December 21-23, 2020) that featured heightened external validity but imperfect randomization, we found suggestive evidence that combining self-interested and prosocial framing may have been more effective at motivating people to sign up for a contact tracing app than self-interested or prosocial framing alone.

Thus, across all of our experiments, we never found self-interested framing to be significantly more effective than prosocial framing. These findings are striking, considering the substantial risks of hospitalization and death posed by COVID-19 to individuals. And they suggest that people are receptive to the suggestion that prevention behaviors can, in additional to conferring personal benefits, also serve to benefit others. In this way, our results imply that prosocial motives - or the desire to appear prosocial — can support prevention behaviors.

We also supported this proposal with correlational analyses. Across all of our survey experiments, we consistently found that the perceived public threat of coronavirus is more strongly associated with prevention intentions than the perceived personal threat. Furthermore, we found that people who behaved prosocially in incentivized economic games conducted years before the pandemic reported greater prevention intentions. While these correlation results cannot provide decisive causal evidence for the influence of prosocial motives, they are robust to demographic controls and provide suggestive evidence that builds on our treatment effects. 
Together, our results thus challenge the hypotheses that self-interested motives are the dominant driver of prevention intentions, and that self-interested appeals are the best messaging strategy. And they suggest that prosociality can play an important role in helping to combat COVID-19.

Our results thus contribute to the body of research demonstrating that both self-interest and prosociality can motivate people to prevent infectious disease spread-including recent work specifically investigating efforts to prevent coronavirus ${ }^{3,43-51}$. And our findings align with evidence that people are moral actors who care for others and are motivated to avoid appearing selfish, and that regard for others is especially strong in the domain of physically aversive outcomes.

It is interesting that we found an advantage for prosocial appeals in our first set of survey experiments, but no differences in the effectiveness of our treatments in our second set of survey experiments. While our data cannot speak to the explanation for this change, one possibility is that the psychology surrounding coronavirus changed meaningfully between March and April 2020, as the pandemic progressed. Regardless, the differences between our sets of results highlight that an important goal for ongoing COVID-19 research is to continue investigating the value of prosocial framing across contexts.

Another important question pertains to the efficacy of prosocial framing across different messaging content. While our results highlight the potential power of prosocial framing, our survey experiments tested two (similar) sets of messages. When designing the treatments used in these experiments, we sought to emphasize the substantial threats posed by coronavirus to both individuals and society. However, different self-interested appeals could potentially be more effective (e.g., if they compellingly suggested that young people are at higher personal risk than most believe). Furthermore, we contrasted concern for oneself with concern for one's community at large; concern for close others (e.g., friends and family) ${ }^{45,49}$ presents an interesting intermediate case that combines self-interested and prosocial motives differently than our Personal+Public treatment did. Thus, to gain a general understanding of the value of selfinterested versus prosocial framing, it is important to investigate a variety of different messaging contexts.

Subsequent to our survey experiments, numerous other research groups have used designs that are conceptually similar to ours (i.e., that investigate the efficacy of prosocial versus self-interested framing). As these other studies were conducted at different times, on different study populations, and using different stimuli and dependent measures, they give some insight into the generalizability of our findings. Taken together, the extant body of work supports the conclusions that prosocial framings can motivate coronavirus prevention intentions, and that there is limited evidence that prosocial framings are less effective than self-interested framings.

More specifically, a few papers provide suggestive evidence that prosocial framing may be more effective than self-interested framing. Across three studies of Americans, Luttrell and Petty (2020) found that, as compared to self-focused messages, subjects perceived other-focused messages to be similarly or more persuasive ${ }^{52}$. They also found that other-focused messages were seen as relatively more persuasive by subjects who saw public health as a moral issue. In another study of Americans, Capraro and Barcelo (2020) measured intentions to wear a face covering across four messaging conditions (emphasizing the threat of the virus to you, your family, your community, or your country), plus a control". They found that the "your community" treatment increased intentions relative to the control, while there were no other significant differences between conditions. Additionally, across one study of Turkish participants and two studies of American participants, Ceylan and Hayran (2021) found that, compared to 
self-interested framing, prosocially framed messages were more persuasive at encouraging social distancing ${ }^{53}$. And finally, in a four-wave study of Japanese participants, Sasaki et al (2021) compared the impact of five different messages (including two other-focused, one self-focused, and one both self- and other-focused), plus a control, on measures of self-reported prevention intentions and behaviors ${ }^{54}$. Overall, their nuanced results suggest that the other-focused messages (and especially other-focused messages that were "gain-framed" rather than "loss-framed") were as or more effective than the self-focused or combined messages. However, the effects of their message treatments were generally less persistent over time, and less positive (and in fact were negative for some measures), in the context of self-reported behavior than prevention intentions; and they only analyze participants who responded to all four survey waves, which has the potential to induce selection effects.

Other papers have failed to demonstrate an advantage for prosocial framing, but have also failed to provide clear evidence that prosocial framing is any less effective than selfinterested framing. In a conceptual replication of our survey experiments among Japanese participants, Miyajima and Murakami (2021) evaluated messages emphasizing the benefits of prevention behaviors for you, others, you and others, or your family; they found that all of these messages increased prevention intentions relative to a control, and that there were no efficacy differences between messaging strategies ${ }^{55}$. In a study of Danish participants, Falco and Zaccagni (2020) measured the impact of four text-message reminders treatments (emphasizing the threat to you, your family, others, or the country), and a control, on intentions to stay at home - and, in a follow-up survey, reports of having stayed at home ${ }^{56}$. They found that only the "you" and "family" treatments increased intentions to stay home, relative to the control, and none of their treatments had a significantly positive effect on self-reported behavior in the follow-up; however, their follow-up survey showed high attrition rates, which has the potential to induce selection effects and undermine causal inference. In a study of French participants, Hacquin et al (2020) manipulated the language of two public health posters across a variety of treatment conditions (including one that used other-focused language and one that used selffocused language) and compared each treatment to a control poster ${ }^{57}$. They found no significant difference in prevention behavior intentions across any of their conditions. And finally, in a study of Americans, Favero and Pedersen (2020) compared four messaging treatments (including one self-focused and three other-focused) to a control and found no significant differences between any of their conditions in intentions to engage in social distancing ${ }^{58}$.

Finally, one study did find some evidence for a relative disadvantage of prosocial framing. In a field experiment of American Facebook users, Banker and Park (2020) evaluated the efficacy of advertisements linking users to recommendations from the CDC website ${ }^{59}$. They found that messages using a "distant" prosocial frame ("protect your community") resulted in lower click-through rates than messages using a self-interested frame ("protect yourself"). However, messages using a "close" prosocial frame ("protect your loved ones") were as effective as self-interested messages.

Thus, a variety of studies have now compared the relative efficacy of prosocial versus self-interested framing across a range of messaging contexts. Several of these studies have provided suggestive evidence for an advantage of prosocial framing, while several others have not found clear differences between the efficacy of these framings. Importantly, one study did find evidence that "distant" prosocial framing was less effective than self-interested framing, although it also found that "close" prosocial framing was no less effective. Together, this body of work is broadly consistent with the conclusions that prosocial framings can be effective, and that 
there is limited evidence for contexts in which they are less effective than self-interested framings.

Our work has important limitations. First, as is common in research investigating disease prevention behavior, we mostly focused on self-reported intentions for prevention behaviors (which may be susceptible to socially desirable responding). It is thus possible that prosociallyframed messaging may not be effective for changing actual behavior. Indeed, the aforementioned studies $^{54,56}$ that measured both intentions (immediately after treatment) and self-reported behavior (at follow-up) highlight that messaging interventions may sometimes be more effective at changing intentions than behavior.

However, it is difficult to draw decisive conclusions from these studies, given that (i) attrition (from the initial survey to the follow-up) can challenge causal inference and (ii) retrospective self-reporting of behavior may not always reliably index actual behavior. Furthermore, despite its imperfect randomization, the field experiment we present in Study 6 provides suggestive evidence that adding prosocial framing to self-interested framing may motivate actual behavior. And abstracting away from COVID-19 specifically, meta-analytic evidence suggests that health interventions that induce changes in intentions do typically translate into changes in behavior ${ }^{60}$.

Moreover, even if social pressure to report prevention intentions did contribute to the effectiveness of prosocial messaging, our results may still be relevant. If prosocial messaging heightens social pressure to report prevention intentions, it may also heighten social pressure for actual behavior. And a great deal of research shows the power of social pressure for promoting cooperation outside the laboratory ${ }^{61}$. Nonetheless, it is critical that future work continue to investigate the impact of prosocially-framed messaging on actual prevention behavior.

Another important limitation of our studies is that we primarily used convenience samples of Americans recruited from Mturk, leaving open questions about the generalizability of our results. In particular, Mturk samples tend to skew younger than the national age distribution, limiting our ability to draw inferences about older people-who are a very important and vulnerable population in the context of COVID-19. That said, we do believe that understanding the motives of young people is also essential: young people tend to be less compliant with prevention behaviors, and thus are a critical target for COVID-19 messaging. Yet future work should nonetheless evaluate prosocially-framed messaging both among representative samples of Americans, and samples of vulnerable populations. In addition to older adults, evidence suggests that people from racial and ethnic minority groups are being disproportionately affected by COVID- $19^{62}$, making them another important study population.

Relatedly, future work should continue to investigate the power of prosocial appeals across cultures. It is interesting that our results highlight the power of prosocial motives, given that the United States is a fairly individualist (rather than collectivist) culture ${ }^{63}$. And it is also interesting that the aforementioned set of related studies serves to broadly support our conclusions, despite sampling subjects from a variety of different countries. Further research should continue to evaluate the efficacy of prosocial framing in different cultural contexts.

\section{Data availability}

The datasets generated during and analysed during the current study are publicly available in the on OSF at https://osf.io/sr4n9/. 


\section{References}

1. Anderson, R. M., Heesterbeek, H., Klinkenberg, D. \& Hollingsworth, T. D. How will countrybased mitigation measures influence the course of the COVID-19 epidemic? The Lancet 395, 931-934 (2020).

2. $\mathrm{Su}, \mathrm{Z}$. et al. Vaccines are not yet a silver bullet: The imperative of continued communication about the importance of COVID-19 safety measures. Brain, Behavior, \& Immunity - Health 12, 100204 (2021).

3. Everett, J. A., Colombatto, C., Chituc, V., Brady, W. J. \& Crockett, M. The effectiveness of moral messages on public health behavioral intentions during the COVID-19 pandemic. (2020).

4. Van Bavel, J. J. et al. Using social and behavioural science to support COVID-19 pandemic response. (2020).

5. Smith, A. The Wealth of Nations: An inquiry into the nature and causes of the Wealth of Nations. (1776).

6. Aunger, R. \& Curtis, V. Behaviour Centred Design: towards an applied science of behaviour change. Health psychology review 10, 425-446 (2016).

7. Jolls, C., Sunstein, C. R. \& Thaler, R. A behavioral approach to law and economics. Stanford law review 1471-1550 (1998).

8. Tidwell, J. B. et al. Effect of a behaviour change intervention on the quality of peri-urban sanitation in Lusaka, Zambia: a randomised controlled trial. The Lancet Planetary Health $\mathbf{3}$, e187-e196 (2019).

9. Aquino, K. \& Reed, I. I. The self-importance of moral identity. J Pers Soc Psychol 83, 1423 (2002).

10. DeSteno, D. Emotional success: The power of gratitude, compassion, and pride. (Houghton Mifflin Harcourt, 2018).

11. Fehr, E. \& Fischbacher, U. The nature of human altruism. Nature 425, 785-791 (2003).

12. Hofmann, W., Wisneski, D. C., Brandt, M. J. \& Skitka, L. J. Morality in everyday life. Science 345, 1340-1343 (2014).

13. Zaki, J. \& Mitchell, J. P. Equitable decision making is associated with neural markers of intrinsic value. Proceedings of the National Academy of Sciences of the United States of America 108, 19761-19766 (2011).

14. Barclay, P. Trustworthiness and competitive altruism can also solve the "tragedy of the commons". Evolution and Human Behavior 25, 209-220 (2004).

15. Boyd, R. \& Richerson, P. J. The evolution of indirect reciprocity. Social Networks 11, 213 236 (1989).

16. Fehr, E. \& Gachter, S. Altruistic punishment in humans. Nature 415, 137-140 (2002).

17. Nowak, M. A. \& Sigmund, K. Evolution of indirect reciprocity. Nature 437, 1291-1298 (2005).

18. Batson, C. D., O’Quin, K., Fultz, J., Vanderplas, M. \& Isen, A. M. Influence of self-reported distress and empathy on egoistic versus altruistic motivation to help. Journal of personality and social psychology 45, 706 (1983).

19. Atanasov, P. D. Risk preferences in choices for self and others: Meta analysis and research directions. Available at SSRN 1682569 (2015). 
20. Stone, E. R., YoonSun, C., Bruine de Bruin, W. \& Mandel, D. R. I can take the risk, but you should be safe: Self-other differences in situations involving physical safety. Judgment and Decision making 8, 250-267 (2013).

21. Davis, A. L., Jehli, N., Miller, J. H. \& Weber, R. A. Generosity across contexts. (2015).

22. Davis, A. L., Miller, J. H. \& Bhatia, S. Are preferences for allocating harm rational? Decision 5, 287 (2018).

23. Story, G. W. et al. Social redistribution of pain and money. Scientific reports 5, 15389 (2015).

24. Crockett, M. J., Kurth-Nelson, Z., Siegel, J. Z., Dayan, P. \& Dolan, R. J. Harm to others outweighs harm to self in moral decision making. Proceedings of the National Academy of Sciences 111, 17320-17325 (2014).

25. Betsch, C., Böhm, R., Korn, L. \& Holtmann, C. On the benefits of explaining herd immunity in vaccine advocacy. Nat Hum Behav 1, 0056 (2017).

26. Boudewyns, V., Turner, M. M. \& Paquin, R. S. Shame-free guilt appeals: testing the emotional and cognitive effects of shame and guilt appeals. Psychology \& Marketing 30, 811-825 (2013).

27. Hershey, J. C., Asch, D. A., Thumasathit, T., Meszaros, J. \& Waters, V. V. The roles of altruism, free riding, and bandwagoning in vaccination decisions. Organizational behavior and human decision processes 59, 177-187 (1994).

28. Li, M., Taylor, E. G., Atkins, K. E., Chapman, G. B. \& Galvani, A. P. Stimulating Influenza Vaccination via Prosocial Motives. PLoS One 11, (2016).

29. Vietri, J. T., Li, M., Galvani, A. P. \& Chapman, G. B. Vaccinating to Help Ourselves and Others. Med Decis Making 32, 447-458 (2012).

30. Hendrix, K. S. et al. Vaccine Message Framing and Parents' Intent to Immunize Their Infants for MMR. PEDIATRICS 134, e675-e683 (2014).

31. Gerend, M. A. \& Barley, J. Human papillomavirus vaccine acceptability among young adult men. Sexually transmitted diseases 36, 58-62 (2009).

32. Isler, O., Isler, B., Kopsacheilis, O. \& Ferguson, E. Limits of the social-benefit motive among high-risk patients: a field experiment on influenza vaccination behaviour. $B M C$ Public Health 20, (2020).

33. Bonafide, K. E. \& Vanable, P. A. Male Human Papillomavirus Vaccine Acceptance Is Enhanced by a Brief Intervention that Emphasizes Both Male-Specific Vaccine Benefits and Altruistic Motives. Sexually Transmitted Diseases 42, 76-80 (2015).

34. Cohen, M. Framing Private Vaccination Behavior as a Public Good A Randomized Trial of Self-and Other-Framed Influenza Vaccination Appeals. (YALE UNIVERSITY New Haven, CT, 2016).

35. Grant, A. M. \& Hofmann, D. A. It's Not All About Me: Motivating Hand Hygiene Among Health Care Professionals by Focusing on Patients. Psychol Sci 22, 1494-1499 (2011).

36. Coppock, A. Generalizing from survey experiments conducted on Mechanical Turk: A replication approach. Political Science Research and Methods 7, 613-628 (2019).

37. Paolacci, G., Chandler, J. \& Ipeirotis, P. G. Running experiments on amazon mechanical turk. Judgment and Decision Making 5, 411-419 (2010).

38. Frederick, S. Cognitive reflection and decision making. Journal of Economic perspectives 19, 25-42 (2005). 
39. Pennycook, G., McPhetres, J., Bago, B. \& Rand, D. G. Attitudes about COVID-19 in Canada, the UK, and the USA: A novel test of political polarization and motivated reasoning. (2020).

40. Arechar, A. A. \& Rand, D. G. Learning to be selfish? A large-scale longitudinal analysis of Dictator games played on Amazon Mechanical Turk. https://osf.io/87e4y (2019) doi:10.31234/osf.io/87e4y.

41. Jordan, J. J. \& Rand, D. G. Signaling when nobody is watching: A reputation heuristics account of outrage and punishment in one-shot anonymous interactions. J Pers Soc Psychol (2019).

42. Jordan, M. \& Rand, D. G. The role of character strengths in economic decision-making. Jordan, MR, \& Rand, DG (2018). The Role of Character Strengths in Economic DecisionMaking. Judgment and Decision making 13, 382-392 (2018).

43. Bilancini, E., Boncinelli, L., Capraro, V., Celadin, T. \& Di Paolo, R. The effect of normbased messages on reading and understanding COVID-19 pandemic response governmental rules. (2020).

44. Bos, B., Drupp, M. A., Meya, J. \& Quaas, M. Moral Suasion and the Private Provision of Public Goods: Evidence from the COVID-19 Pandemic. Available at SSRN 3611579 (2020).

45. Capraro, V.\& Barcelo, H. The effect of messaging and gender on intentions to wear a face covering to slow down COVID-19 transmission. https://osf.io/tg7vz (2020) doi:10.31234/osf.io/tg7vz.

46. Heffner, J., Vives, M. L. \& FeldmanHall, O. Emotional responses to prosocial messages increase willingness to self-isolate during the COVID-19 pandemic. (2020).

47. Pfattheicher, S., Nockur, L., Böhm, R., Sassenrath, C. \& Petersen, M. B. The emotional path to action: Empathy promotes physical distancing during the COVID-19 pandemic. (2020).

48. Qian, K. \& Yahara, T. Mentality and behavior in COVID-19 emergency status in Japan: Influence of personality, morality and ideology. PloS one 15, e0235883 (2020).

49. Raihani, N. \& de-Wit, L. Factors Associated With Concern, Behaviour \& Policy Support in Response to SARS-CoV-2. (2020).

50. van Rooij, B. et al. Compliance with COVID-19 Mitigation Measures in the United States. https://osf.io/qymu3 (2020) doi:10.31234/osf.io/qymu3.

51. Yang, L. \& Ren, Y. Moral Obligation, Public Leadership, and Collective Action for Epidemic Prevention and Control: Evidence from the Corona Virus Disease 2019 (COVID19) Emergency. International Journal of Environmental Research and Public Health 17, 2731 (2020).

52. Luttrell, A. \& Petty, R. E. Evaluations of self-focused versus other-focused arguments for social distancing: An extension of moral matching effects. Social Psychological and Personality Science 1948550620947853 (2020).

53. Ceylan, M. \& Hayran, C. Message Framing Effects on Individuals' Social Distancing and Helping Behavior During the COVID-19 Pandemic. Frontiers in psychology 12, 663 (2021).

54. Sasaki, S., Kurokawa, H. \& Ohtake, F. Effective but fragile? Responses to repeated nudgebased messages for preventing the spread of COVID-19 infection. The Japanese Economic Review 1-38 (2021).

55. Miyajima, T. \& Murakami, F. Self-interested framed and prosocially framed messaging can equally promote COVID-19 prevention intention: A replication and extension of Jordan et al.'s study (2020) in the Japanese context. Frontiers in Psychology 12, (2021). 
56. Falco, P. \& Zaccagni, S. Promoting social distancing in a pandemic: Beyond the good intentions. (2020).

57. Hacquin, A.-S., Mercier, H. \& Chevallier, C. Improving preventive health behaviors in the COVID-19 crisis: a messaging intervention in a large nationally representative sample. (2020).

58. Favero, N. \& Pedersen, M. J. How to encourage "Togetherness by Keeping Apart" amid COVID-19? The ineffectiveness of prosocial and empathy appeals. Journal of Behavioral Public Administration 3, (2020).

59. Banker, S. \& Park, J. Evaluating prosocial COVID-19 messaging frames: Evidence from a field study on Facebook. Judgment and Decision Making 15, 1037-1043 (2020).

60. Webb, T. L. \& Sheeran, P. Does changing behavioral intentions engender behavior change? A meta-analysis of the experimental evidence. Psychological bulletin 132, 249 (2006).

61. Kraft-Todd, G., Yoeli, E., Bhanot, S. \& Rand, D. Promoting cooperation in the field. Current Opinion in Behavioral Sciences 3, 96-101 (2015).

62. Wilder, J. M. The Disproportionate Impact of COVID-19 on Racial and Ethnic Minorities in the United States. Clin Infect Dis (2020) doi:10.1093/cid/ciaa959.

63. Hofstede, G. Culture's consequences: International differences in work-related values. vol. 5 (sage, 1984).

\section{Acknowledgements}

We thank Matthew Higgins for helpful research assistance. Additionally, the flyers used in our survey were based on designs by Syon Bhanot and the Busara Center for Behavioral Economics. 


\section{Supplementary information \\ for \\ Don't get it or don't spread it: Comparing self-interested versus prosocial motivations for COVID-19 prevention behaviors}

Jillian J. Jordan, Erez Yoeli, and David G. Rand

1. Individual difference measures collected in Studies 1 $1-3$................................................... 2

2. Procedure for calculating q-values................................................................................... 2

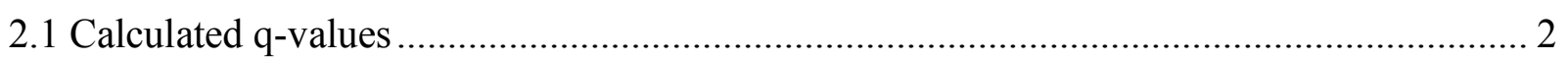

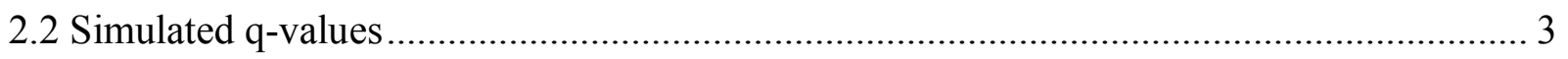

3. Procedure for accounting for data "peeking" in Studies 1-2 ........................................... 4

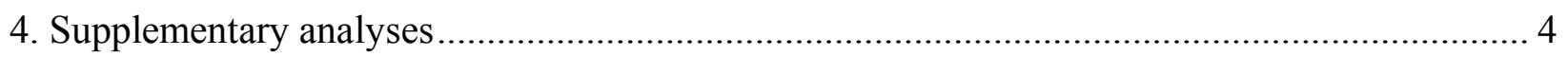

4.1 Association between prevention intentions and age across Studies 1-4 f....................... 4

4.2 Analyses of social distancing intentions in Study 1 ................................................ 5

4.3 Heterogeneity of treatment effects across prevention behaviors in Studies 1-2 ................ 6

4.4 Heterogeneity of treatment effects across individuals in Studies $1-2$.............................. 8

4.5 Perceived public and personal threat of coronavirus across conditions in Studies 1-3 ....... 9

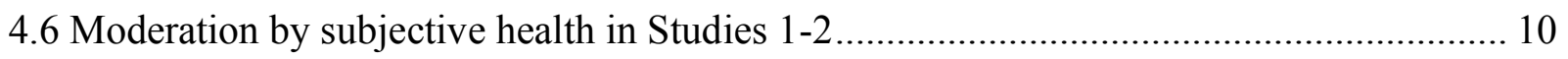

4.7 Did the relative effectiveness of treatments depend on Time 1 prevention intentions in

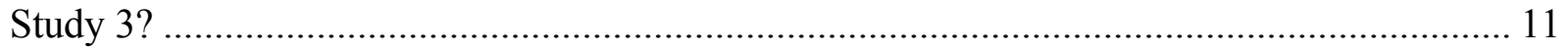

4.8 Analyses of "English check" in Studies 1-2 and attention checks in Study 3 ................. 12

4.9 Alternative choices about public and personal threat items in Study 4 ........................... 13

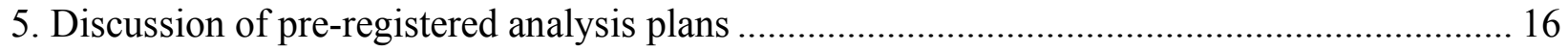

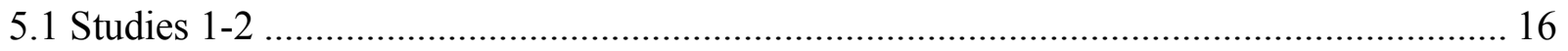

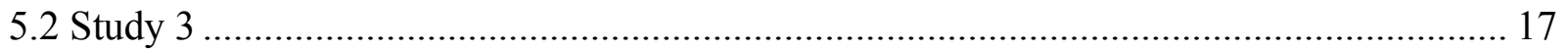

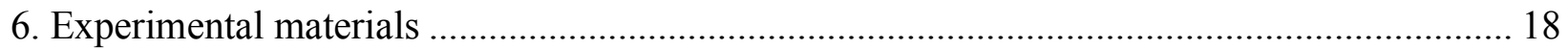

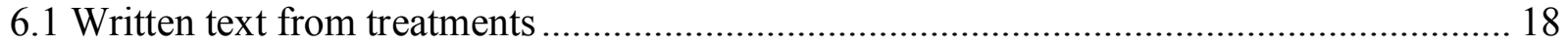

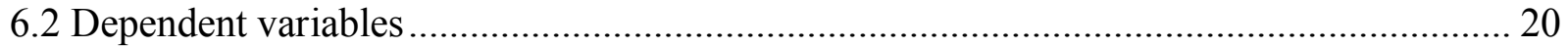

6.3 Perceived public and personal threat of coronavirus ................................................. 24

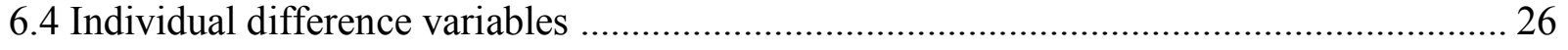

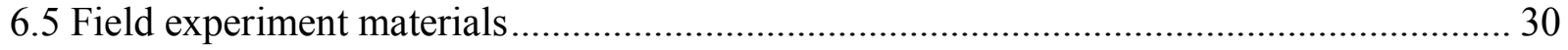




\section{Individual difference measures collected in Studies 1-3}

As mentioned in the main text, in Studies 1-3 we collected a set of individual difference variables. Here, we describe these variables; for exact wording for all questions, see Section 6.

In Studies 1-2, we measured, in a fixed order, age, gender, level of education, zip code, subjective health, number of pre-existing health conditions (from a list of conditions we specified), income bracket, political ideology (via three questions about political party identification, position on social issues, and position on fiscal issues), and previous exposure to information about COVID-19. Next, we presented subjects with a three-item Cognitive Reflection Task. Finally, we asked subjects to answer a simple analogy question and write a few sentences about their plans for the day; these measures were designed to screen for subjects who did not speak English (see Section 4.8 for analyses).

In Studies 3a-c, we measured the same set of individual differences variables as in Studies 1-2, with the exceptions that that we (i) added measures of race and previous participation in surveys about COVID-19, (ii) only included one measure of political ideology (political party identification), and (iii) replaced our "English check" questions with two attention check questions (in which the question text instructed attentive subjects to select specific answer choices; see Section 4.8 for analyses).

Study 3d was identical to Studies 3a-c, with the exception that, for brevity, we omitted our measures of pre-existing health conditions, zip code, and previous exposure to information about COVID-19, as well as both attention checks and the cognitive reflection task.

\section{Procedure for calculating q-values}

As discussed in the main text, in Studies 1-2 we tested three treatments and measured two dependent variables (DVs), creating six possible comparisons, and in Study 3 we tested three treatments and measured one DV, creating three possible comparisons. Thus, in addition to reporting p-values for these comparisons, we also report q-values, which indicate the probability of making at least one false discovery across the set of comparisons when rejecting the null hypothesis for any result with an equal or smaller q-value. We note that in Studies 1-2, we do not account for our analyses of subjects for whom we measured our dependent variables first as a separate set of comparisons, because we simply include these analyses as a robustness check (and not an independent opportunity to support a given hypothesis).

For both sets of studies, we report calculated q-values (reported as $q_{c}$ ), derived from analytical calculations that conservatively assume that the tests for all six comparisons are independent from each other. And for Studies 1-2 only, we also report simulated q-values (reported as $q_{s}$ ), derived from simulations of our actual data that take into account the nonindependence between some tests. (As noted in the main text, we did not generate simulated qvalues for Study 3 because the results are clearly definitive in supporting the conclusions that all treatments were effective, and there were no meaningful differences in effectiveness between treatments.)

Here we provide more details about how we derived these q values; full code to reproduce our simulations is available at https://osf.io/sr4n9/.

\subsection{Calculated q-values}

To calculate $q-v a l u e s$ analytically, we compute $q$ as $1-(1-p)^{\wedge} n$, where $n$ is the number of comparisons (i.e., six in Studies 1-2 and three in Study 3). Our calculated q-values thus represent 
the probability of making at least one false discovery across the full set of tests at the relevant pvalue threshold, assuming that all tests are independent from each other.

\subsection{Simulated q-values}

For Studies 1-2, we also compute simulated q-values, which account for the fact that the assumption of independence is overly conservative, given that (i) our two dependent variables are correlated in Study $1(r=.59, p<.001)$, and (ii) the three pairwise comparisons are not independent.

Each of our simulations estimate the expected probability of making at least one false discovery across 10,000 simulation rounds. In each round, we (i) randomly sample observations (with replacement) from our data, (ii) assign each observation to a random condition (thus forcing the null hypothesis to be true), and then (iii) conduct the six relevant tests. We then calculate simulated q-values for a given $\mathrm{p}$-value as the proportion of simulation rounds in which the minimum simulated p-value across the six tests is smaller than the given p-value. Below, we provide more detail about our simulations; note that we refer to prevention intentions (which we measured in both studies) as DV1, and social distancing intentions (which we measured only in Study 1) as DV2.

First, we describe the process we use to simulate q-values for the reported comparisons of our treatments to the control condition in Study 1, for both DV1 (main text Table 1) and DV2 (SOM Table S1). In each simulation round, we (i) sample from all conditions of our Study 1 data, with $n$ equal to the number of observations across all conditions of Study 1, and then (ii) conduct six tests by comparing each treatment to the control for each DV.

Next, we describe the process we use to simulate q-values for the reported pairwise comparisons between our treatments. To conduct pairwise comparisons between treatments for DV1 (main text Table 2), we analyzed pooled data from Studies 1 and 2 (because both studies measured DV1). In contrast, to conduct pairwise comparisons between treatments for DV2 (SOM Table S2), we analyzed data from Study 1 only (because only Study 1 measured DV2). Accordingly, we conduct two distinct simulations to generate q-values for our analyses of DV1 and DV2, respectively. However, because there are six total comparisons across our two DVs, we use each of these simulations to determine the probability of making at least one false discovery across six tests; thus, each simulation samples data from both DVs.

Therefore, to generate q-values for our pairwise comparisons for DV2, in each simulation round, we (i) sample from the treatment conditions of our Study 1 data, with $n$ equal to the number of observations across the treatment conditions of Study 1, and then (ii) conduct six tests by comparing each pair of treatments for each DV.

In contrast, to generate q-values for our pairwise comparisons for DV1, in each simulation round, we (i) sample from the treatment conditions of our Study 1 data, with $n$ equal to the number of observations across the treatment conditions of Studies 1 and 2, and then (iii) conduct six tests by comparing each pair of treatments for each DV. We note that we sample exclusively from the Study 1 data (despite actually having analyzed data from both studies) because we did not collect DV2 in Study 2; thus, only our Study 1 data allow us to account for the correlation between DV1 and DV2 when simulating the probability of false discovery across the six tests. While the distributions of DV1 values are similar in Studies 1 vs. 2, mean values of DV1 are slightly higher in Study 2, so this approach is only approximate. We also note that to simulate the precise approach we used in our analyses, our simulations categorize observations 
as belonging to "Study 1" or "Study 2" (with the sample sizes corresponding to the actual sizes of Study 1 and Study 2), and each test includes a "study" dummy in the model.

Finally, we note that when we report simulated q-values for subpopulations in our data (specifically, subjects for whom we measured our dependent variables first, in the main text, and subjects reporting above-median subjective health, in Section 4.6), they come from independent simulations that sample exclusively from those subpopulations (and sample proportionately fewer observations).

\section{Procedure for accounting for data "peeking" in Studies 1-2}

In the main text, we show that the Public treatment was more effective than the Personal treatment at increasing prevention intentions via an analysis of the pooled data from Studies 1 and 2. As noted in the main text, our analyses of the pooled data across studies can be conceptualized as analyses of one study in which we "peeked" at the data after an initial collection, which can inflate type-I error rate. However, our conclusion that the Public treatment was more effective than the Personal treatment is robust to accounting for peeking.

Using Sagarin, Ambler, and Lee's (2014) method to evaluate augmented datasets that are based on peeking at marginally significant results, we calculated that, to maintain an actual typeI error rate of .05, it is necessary to evaluate statistical significance in our pooled dataset using an alpha threshold ranging from a "best-case scenario" of .0471 to a "worst-case scenario" of .0281 (for our analysis of all subjects; for our robustness check analysis of subjects for whom we measured our dependent variables first, the range is .049999 to .0283). We report a range, because the required alpha threshold depends on the maximum p-value observed in Study 1 for which we would have conducted Study 2 rather than declaring the initial results non-significant; this could range from a "best-case scenario" of the p-value observed in Study 1 (.066 among all subjects and .029 among subjects for whom we measured our DVs first) to a "worst-case scenario" of 1. Looking to the Public vs. Personal comparison in the main text Table 2, we note that all p- and q-values are below these "worst-case scenario" thresholds, reflecting that our results are still significantly significant after accounting for peeking.

\section{Supplementary analyses}

\subsection{Association between prevention intentions and age across Studies 1-4}

As noted in the main text, Mturk samples tend to skew young (as compared to the U.S. national age distribution). Although not representative, this skew may make Mturk samples valuable for evaluating the effectiveness of messaging, given that young people are less likely to engage in prevention behaviors. Here, we support this claim via an analysis of the association between age and prevention intentions.

First, we report an aggregate analysis of our Mturk studies (i.e., Studies 1-3, total $n=$ $6,161)$. To conduct this analysis, we used our composite measure of prevention intentions from Studies 1-2 and our composite measure of Time 1 prevention intentions from Studies 3a-d. We found a significant positive association between prevention intentions and age (without controls $B=.10, t=7.93, p<.001$, in a model with controls for gender, education, race, income, and political party affiliation $B=.10, t=8.04, p<.001)$. We note that we also find similar results when using Time 2 prevention intentions from Studies 3a-d (without controls $B=.11, t=8.67, p$ $<.001$, with demographic controls $B=.11, t=8.61, p<.001)$. We also note that in our models 
with controls, because race was only measured in Study 3, we recoded our dummies for each racial category to include a third value for missing data.

Finally, we report an analysis of our more representative Prolific study (i.e., Study $4, n=$ 748). In this sample, we likewise found a significant positive association between prevention intentions and age (without controls $B=.13, t=3.52, \mathrm{p}<0.001$, with demographic controls $B=$ $.15, t=4.05, \mathrm{p}<0.001)$.

\subsection{Analyses of social distancing intentions in Study 1}

As reported in the main text, we do not find robust evidence of treatment effects, or differences between treatments, on our measure of composite social distancing intentions collected in Study 1. In Table S1, we report the effects of each of our treatments (relative to the control condition), and in Table S2, we report pairwise comparisons between each treatment pair.

\begin{tabular}{|c|c|c|}
\hline & All subjects $(n=985)$ & Dependant variables first $(n=505)$ \\
\hline Personal & $\begin{array}{c}\text { Control }=66.50[63.69,69.32] \\
\text { Personal }=69.56[66.87,72.25] \\
\mathbf{b}=\mathbf{3 . 0 6}, \mathbf{t}=\mathbf{1 . 5 8}, \mathbf{d}=\mathbf{0 . 1 4} \\
\mathrm{p}=.114, \mathrm{q}_{\mathrm{c}}=.518, \mathrm{q}_{\mathrm{s}}=.419\end{array}$ & $\begin{array}{c}\text { Control }=62.91[58.74,67.07] \\
\text { Personal }=68.10[64.34,71.85] \\
\mathbf{b}=\mathbf{5 . 1 9}, \mathbf{t}=\mathbf{1 . 9 2}, \mathbf{d}=\mathbf{0 . 2 4} \\
\mathrm{p}=.056, \mathrm{q}_{\mathrm{c}}=.292, \mathrm{q}_{\mathrm{s}}=.238\end{array}$ \\
\hline Public & $\begin{array}{c}\text { Control }=66.50[63.69,69.32] \\
\text { Public }=69.47[66.87,72.08] \\
\mathbf{b}=\mathbf{2 . 9 7}, \mathbf{t}=\mathbf{1 . 5 3}, \mathbf{d}=\mathbf{0 . 1 4} \\
\mathrm{p}=.127, \mathrm{q}_{\mathrm{c}}=.557, \mathrm{q}_{\mathrm{s}}=.456\end{array}$ & $\begin{array}{c}\text { Control }=62.91[58.74,67.07] \\
\text { Public }=68.59[64.85,72.32] \\
\mathbf{b}=\mathbf{5 . 6 8}, \mathbf{t}=\mathbf{2 . 0 7}, \mathbf{d}=\mathbf{0 . 2 6} \\
\mathrm{p}=.039, \mathrm{q}_{\mathrm{c}}=.210, \mathrm{q}_{\mathrm{s}}=.172\end{array}$ \\
\hline $\begin{array}{c}\text { Personal + } \\
\text { Public }\end{array}$ & $\begin{array}{c}\text { Control }=66.50[63.69,69.32] \\
\text { Personal+Public }=69.60[66.93,72.27] \\
\mathbf{b}=\mathbf{3 . 1 0}, \mathbf{t}=\mathbf{1 . 5 8}, \mathbf{d}=\mathbf{0 . 1 4}, \\
\mathrm{p}=.114, \mathrm{q}_{\mathrm{c}}=.516, \mathrm{q}_{\mathrm{s}}=.416\end{array}$ & $\begin{array}{c}\text { Control }=62.91[58.74,67.07], \\
\text { Personal }+ \text { Public }=71.83[68.54,75.11], \\
\mathbf{b}=\mathbf{8 . 9 2}, \mathbf{t}=\mathbf{3 . 2 3}, \mathbf{d}=\mathbf{0 . 4 2}, \\
\mathrm{p}=.001, \mathrm{q}_{\mathrm{c}}=.008, \mathrm{q}_{\mathrm{s}}=.006\end{array}$ \\
\hline
\end{tabular}

Table S1. Treatment effects on social distancing intentions. We compare each of our treatments to the control in Study 1. For each treatment, we report mean prevention intentions (with 95\% CIs) in the treatment and control conditions, and the treatment effect (both among all subjects, and subjects for whom we measured our dependent variables first). 


\begin{tabular}{|c|c|c|}
\hline & All subjects $(n=739)$ & Dependant variables first $(n=\mathbf{3 8 8})$ \\
\hline $\begin{array}{l}\text { Public vs. } \\
\text { Personal }\end{array}$ & $\begin{array}{c}\text { Public }=69.47[66.87,72.08] \\
\text { Personal }=69.56[66.87,72.25] \\
\mathbf{b}=\mathbf{- 0 . 0 9}, \mathbf{t}=\mathbf{- 0 . 0 5}, \mathbf{d}=\mathbf{- 0 . 0 0} \\
\mathrm{p}=.963, \mathrm{q}_{\mathrm{c}}=1.000, \mathrm{q}_{\mathrm{s}}=1.000\end{array}$ & $\begin{array}{c}\text { Public }=68.59[64.85,72.32] \\
\text { Personal }=68.10[64.34,71.85] \\
\mathbf{b}=\mathbf{0 . 4 9}, \mathbf{t}=\mathbf{0 . 1 9}, \mathbf{d}=\mathbf{0 . 0 2} \\
\mathrm{p}=.851, \mathrm{q}_{\mathrm{c}}=1.000, \mathrm{q}_{\mathrm{s}}=1.000\end{array}$ \\
\hline $\begin{array}{c}\text { Public vs. } \\
\text { Personal+Public }\end{array}$ & $\begin{array}{c}\text { Public }=69.47[66.87,72.08] \\
\text { Personal }+ \text { Public }=69.60[66.93,72.27] \\
\mathbf{b}=\mathbf{- 0 . 1 3 , t}=\mathbf{- 0 . 0 7 ,} \mathbf{d}=\mathbf{- 0 . 0 1}, \\
\mathrm{p}=.948, \mathrm{q}_{\mathrm{c}}=1.000, \mathrm{q}_{\mathrm{s}}=1.000\end{array}$ & $\begin{array}{c}\text { Public }=68.59[64.85,72.32], \\
\text { Personal+Public }=71.83[68.54,75.11] \\
\mathbf{b}=\mathbf{- 3 . 2 4}, \mathbf{t}=\mathbf{- 1 . 2 3}, \mathbf{d}=\mathbf{- 0 . 1 5}, \\
\mathrm{p}=.220, \mathrm{q}_{\mathrm{c}}=.774, \mathrm{q}_{\mathrm{s}}=.638\end{array}$ \\
\hline $\begin{array}{c}\text { Pesonal vs. } \\
\text { Personal+Public }\end{array}$ & $\begin{array}{c}\text { Personal }=69.56[66.87,72.25] \\
\text { Personal+Public }=69.60[66.93,72.27] \\
\mathbf{b}=\mathbf{- 0 . 0 4}, \mathbf{t}=\mathbf{- 0 . 0 2}, \mathbf{d}=\mathbf{- 0 . 0 0}, \\
\mathrm{p}=.985, \mathrm{q}_{\mathrm{c}}=1.000, \mathrm{q}_{\mathrm{s}}=1.000\end{array}$ & $\begin{array}{l}\text { Personal }=68.10[64.34,71.85], \\
\text { Personal+Public }=71.83[68.54,75.11] \\
\mathbf{b}=\mathbf{- 3 . 7 3 ,} \mathbf{t}=\mathbf{- 1 . 4 3 ,} \mathbf{d}=\mathbf{- 0 . 1 8}, \\
\mathrm{p}=.154, \mathrm{q}_{\mathrm{c}}=.632, \mathrm{q}_{\mathrm{s}}=.503\end{array}$ \\
\hline
\end{tabular}

Table S2. Comparisons between treatments on social distancing intentions. We conduct pairwise comparisons across the treatment conditions of Study 1 (both among all subjects, and subjects for whom we measured our dependent variables first).

\subsection{Heterogeneity of treatment effects across prevention behaviors in Studies 1-2}

Here, we investigate whether there is meaningful heterogeneity of treatment effects across prevention behaviors in Studies 1-2. As discussed in the main text, our primary analyses of prevention intentions investigated composite prevention intentions, computed by averaging intentions to engage in our set of eleven individual prevention behaviors. Here, we consider this set of behaviors individually.

In Figure S1A, we plot overall treatment effects (i.e., effects of a "treatment vs. control" dummy) on each individual prevention behavior in Study 1. In Figure S1B, we plot effects of the Public treatment, relative to the other two treatments, on each individual prevention behavior across Studies 1 and 2. We show results both among all subjects, and subjects for whom we measured our dependent variables first. 

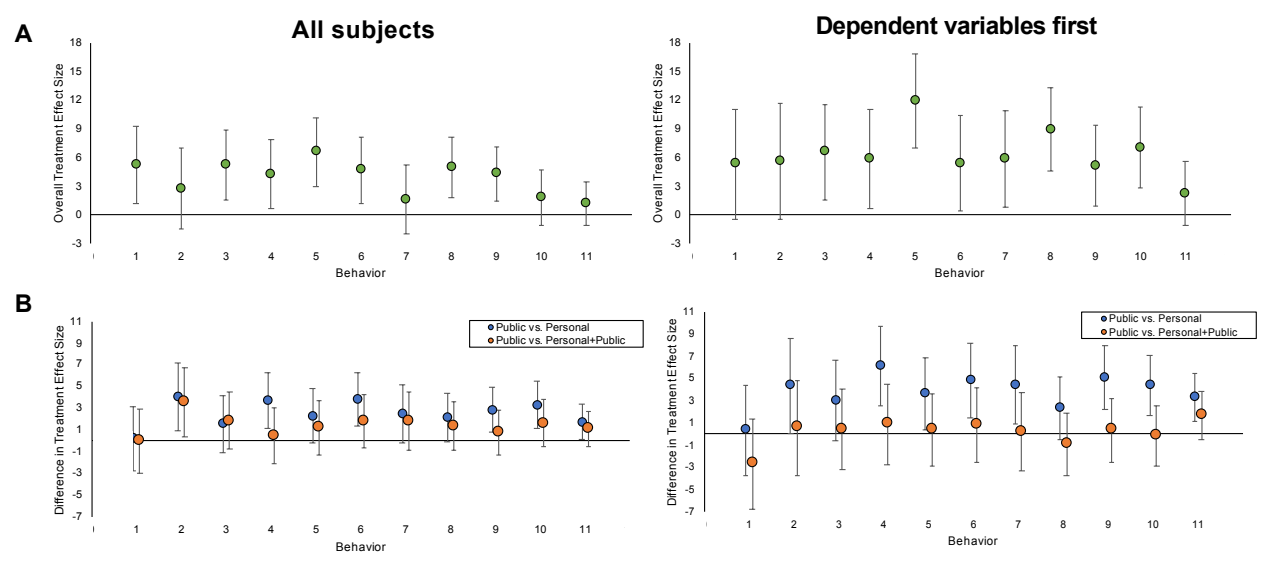

Behavior legend

$$
\begin{aligned}
& \text { 1: } \text { Stock up on cleaning supplies }(\text { Baseline }=64.94) \\
& \text { 2: Wash hands } 10 x \text { a day }(\text { Baseline }=68.22) \\
& \text { 3: Purchase food reserves and meds }(\text { Baseline }=70.87) \\
& \text { 4: Stay home whenever possible }(\text { Baseline }=73.85) \\
& \text { 5: Stop hugging (Baseline }=75.20) \\
& \text { 6: Try hardest to avoid touching face }(\text { Baseline }=75.43) \\
& \text { 7: Wash hands as much as possible }(\text { Baseline }=75.94) \\
& \text { 8: Stop shaking hands }(\text { Baseline }=81.07) \\
& \text { 9: Stay home if even a little bit sick }(\text { Baseline }=81.69) \\
& \text { 10: Wash hands more often }(\text { Baseline }=83.89) \\
& \text { 11: Cover cough and sneeze }(\text { Baseline }=89.39)
\end{aligned}
$$

Figure S1. Treatment effects on individual prevention behaviors. (A) Overall treatment effects on individual behaviors. Shown are the aggregated effects of our three treatments, as compared to the control condition (green dots), in Study 1 (among all subjects, $n=988$, and subjects for whom we measured our dependent variables first, $n=506$ ). (B) Effects of the Public treatment on individual behaviors. Shown are the relative effects of the Public treatment, as compared to the Personal treatment (blue dots) and Personal +Public treatment (orange dots), across the treatment conditions of Studies 1 and 2 combined (among all subjects, $n=1930$, and subjects for whom we measured our dependent variables first, $n=981)$. Behaviors are organized in ascending order by "baseline" intentions to engage in the behavior (defined by mean intentions in the control condition of Study 1, among all subjects), and this value is also reported in the behavior legend. Error bars are 95\% CIs.

Figure S1 reveals that our overall treatment effect, and the advantage of our Public treatment relative to other treatments, are relatively robust across individual prevention behaviors. Confirming this visual impression, we find no significant heterogeneity across individual prevention behaviors. To test for heterogeneity for each condition contrast in Figure S1, we reshaped our data to long format (with one observation per prevention intention item per subject). We then performed a joint significance test on the interaction terms between a dummy for the relevant condition contrast, and dummies for each of the prevention intention items (with robust standard errors clustered on subject).

In analyses of all subjects, we found no significant heterogeneity across behaviors for (i) the contrast between treatments and control in Study $1, F(10,987)=1.38, p=.183$, or (ii) the contrasts between Public and Personal, $F(10,1929)=1.51, p=.131$, or Public and Personal+Public, $F(10,1929)=0.91, p=.519$, across Studies 1 and 2. Likewise, in analyses of all subjects for whom we measured our dependent variables first, we found no significant heterogeneity across behaviors for (i) the contrast between treatments and control in Study 1, $F(10,505)=1.65, p=.090$, or (ii) the contrasts between Public and Personal, $F(10,980)=1.54, p$ $=.121$, or Public and Personal+Public, $F(10,980)=1.31, p=.218$, across Studies 1 and 2 . 
behaviors.

Thus, we find no evidence of heterogeneity in treatment effects across individual

\subsection{Heterogeneity of treatment effects across individuals in Studies 1-2}

Next, we investigate potential heterogeneity of treatment effects across individuals in Studies 1-2. Specifically, in Table S3, we report a set of exploratory analyses investigating whether each of our individual difference variables moderate our treatment effects.

\begin{tabular}{|c|c|c|c|c|c|c|}
\hline & \multicolumn{3}{|c|}{ All subjects } & \multicolumn{3}{|c|}{ Dependant variables first } \\
\hline & $\begin{array}{c}\text { All treatments vs. } \\
\text { Control } \\
\text { in Study } 1 \\
\end{array}$ & $\begin{array}{c}\text { Public vs. } \\
\text { Personal } \\
\text { in Studies 1-2 } \\
\end{array}$ & $\begin{array}{c}\text { Public vs. } \\
\text { Pesonal + Public } \\
\text { in Studies 1-2 } \\
\end{array}$ & $\begin{array}{c}\text { All treatments vs. } \\
\text { Control } \\
\text { in Study } 1 \\
\end{array}$ & $\begin{array}{c}\text { Public vs. } \\
\text { Personal } \\
\text { in Studies 1-2 } \\
\end{array}$ & $\begin{array}{c}\text { Public vs. } \\
\text { Pesonal + Public } \\
\text { in Studies 1-2 }\end{array}$ \\
\hline Age & $\begin{array}{c}\mathbf{0 . 1 7 0} \\
(0.118)\end{array}$ & $\begin{array}{c}\mathbf{0 . 1 2 0} \\
(0.0903)\end{array}$ & $\begin{array}{c}\mathbf{0 . 0 6 8 8} \\
(0.0906)\end{array}$ & $\begin{array}{c}\mathbf{0 . 2 2 8} \\
(0.177)\end{array}$ & $\begin{array}{c}\mathbf{0 . 1 6 9} \\
(0.127)\end{array}$ & $\begin{array}{c}\mathbf{- 0 . 0 2 1 8} \\
(0.129)\end{array}$ \\
\hline Male & $\begin{array}{l}\mathbf{- 0 . 0 3 6 5} \\
(0.0717)\end{array}$ & $\begin{array}{r}\mathbf{- 0 . 0 3 5 2} \\
(0.0440)\end{array}$ & $\begin{array}{l}\mathbf{- 0 . 0 0 1 5 0} \\
(0.0444)\end{array}$ & $\begin{array}{l}\mathbf{- 0 . 1 3 0} \\
(0.102)\end{array}$ & $\begin{array}{l}\mathbf{0 . 0 0 7 7 1} \\
(0.0641)\end{array}$ & $\begin{array}{l}\mathbf{- 0 . 0 2 5 9} \\
(0.0624)\end{array}$ \\
\hline College degree & $\begin{array}{c}\mathbf{0 . 0 8 4 8} \\
(0.0762)\end{array}$ & $\begin{array}{c}\mathbf{0 . 0 4 1 0} \\
(0.0498)\end{array}$ & $\begin{array}{c}\mathbf{0 . 0 2 4 8} \\
(0.0477)\end{array}$ & $\begin{array}{c}\mathbf{0 . 1 5 5} \\
(0.109)\end{array}$ & $\begin{array}{c}\mathbf{0 . 0 3 5 2} \\
(0.0710)\end{array}$ & $\begin{array}{c}\mathbf{0 . 0 5 6 0} \\
(0.0668)\end{array}$ \\
\hline Subjective health & $\begin{array}{r}\mathbf{- 0 . 0 9 2 2} \\
(0.169)\end{array}$ & $\begin{array}{c}\mathbf{0 . 2 5 1} \\
(0.141)\end{array}$ & $\begin{array}{l}\mathbf{0 . 3 2 6 *} \\
(0.135)\end{array}$ & $\begin{array}{l}\mathbf{- 0 . 0 2 2 5} \\
(0.221)\end{array}$ & $\begin{array}{c}\mathbf{0 . 1 0 2} \\
(0.198)\end{array}$ & $\begin{array}{c}\mathbf{0 . 2 0 0} \\
(0.187)\end{array}$ \\
\hline Pre-existing health conditions & $\begin{array}{l}\mathbf{- 0 . 0 3 3 9} \\
(0.0644)\end{array}$ & $\begin{array}{l}\mathbf{- 0 . 0 4 9 6} \\
(0.0375)\end{array}$ & $\begin{array}{l}\mathbf{- 0 . 0 4 5 5} \\
(0.0366)\end{array}$ & $\begin{array}{l}\mathbf{- 0 . 0 5 5 5} \\
(0.0939)\end{array}$ & $\begin{array}{l}\mathbf{- 0 . 0 3 9 9} \\
(0.0591)\end{array}$ & $\begin{array}{l}\mathbf{0 . 0 0 7 7 3} \\
(0.0546)\end{array}$ \\
\hline Income & $\begin{array}{c}\mathbf{0 . 1 6 6} \\
(0.105)\end{array}$ & $\begin{array}{c}\mathbf{0 . 1 4 2} \\
(0.0746)\end{array}$ & $\begin{array}{c}\mathbf{0 . 1 8 5} * \\
(0.0733)\end{array}$ & $\begin{array}{c}\mathbf{0 . 2 5 1} \\
(0.147)\end{array}$ & $\begin{array}{c}\mathbf{0 . 1 2 3} \\
(0.106)\end{array}$ & $\begin{array}{c}\mathbf{0 . 1 7 9} \\
(0.103)\end{array}$ \\
\hline $\begin{array}{c}\text { Conservative (vs. liberal) } \\
\text { political ideology: } \\
\text { Party identification }\end{array}$ & $\begin{array}{l}\mathbf{- 0 . 0 2 9 2} \\
(0.0867)\end{array}$ & $\begin{array}{l}\mathbf{- 0 . 0 6 3 4} \\
(0.0606)\end{array}$ & $\begin{array}{l}\mathbf{- 0 . 1 2 8 *} \\
(0.0594)\end{array}$ & $\begin{array}{r}\mathbf{- 0 . 0 0 1 2 2} \\
(0.120)\end{array}$ & $\begin{array}{c}\mathbf{- 0 . 1 4 6} \\
(0.0895)\end{array}$ & $\begin{array}{c}\mathbf{- 0 . 1 4 3} \\
(0.0842)\end{array}$ \\
\hline $\begin{array}{l}\text { Conservative (vs. liberal) } \\
\text { political ideology: } \\
\text { Social issues }\end{array}$ & $\begin{array}{l}\mathbf{- 0 . 0 5 3 2} \\
(0.0864)\end{array}$ & $\begin{array}{l}\mathbf{- 0 . 0 4 5 1} \\
(0.0579)\end{array}$ & $\begin{array}{l}\mathbf{- 0 . 0 1 9 8} \\
(0.0569)\end{array}$ & $\begin{array}{l}\mathbf{- 0 . 0 1 9 4} \\
(0.120)\end{array}$ & $\begin{array}{c}\mathbf{- 0 . 1 3 3} \\
(0.0835)\end{array}$ & $\begin{array}{l}\mathbf{- 0 . 0 6 1 6} \\
(0.0802)\end{array}$ \\
\hline $\begin{array}{c}\text { Conservative (vs. liberal) } \\
\text { political ideology: } \\
\text { Fiscal issues }\end{array}$ & $\begin{array}{l}\mathbf{- 0 . 0 5 1 2} \\
(0.0910)\end{array}$ & $\begin{array}{r}\mathbf{0 . 0 3 3 4} \\
(0.0623)\end{array}$ & $\begin{array}{l}\mathbf{- 0 . 0 0 5 0 0} \\
(0.0616)\end{array}$ & $\begin{array}{l}\mathbf{0 . 0 8 8 8} \\
(0.128)\end{array}$ & $\begin{array}{l}\mathbf{- 0 . 0 3 7 7} \\
(0.0898)\end{array}$ & $\begin{array}{l}\mathbf{- 0 . 0 4 7 5} \\
(0.0872)\end{array}$ \\
\hline $\begin{array}{l}\text { Previous exposure to } \\
\text { COVID info }\end{array}$ & $\begin{array}{l}-\mathbf{0 . 1 2 0} \\
(0.139)\end{array}$ & $\begin{array}{c}\mathbf{0 . 0 6 4 9} \\
(0.0933)\end{array}$ & $\begin{array}{c}-\mathbf{0 . 1 1 6} \\
(0.0953)\end{array}$ & $\begin{array}{l}\mathbf{- 0 . 0 6 3 2} \\
(0.188)\end{array}$ & $\begin{array}{l}-\mathbf{0 . 2 1 0} \\
(0.130)\end{array}$ & $\begin{array}{l}\mathbf{- 0 . 2 3 7} \\
(0.136)\end{array}$ \\
\hline CRT score & $\begin{array}{c}\mathbf{0 . 0 0 0 5 0 1} \\
(0.0747)\end{array}$ & $\begin{array}{c}\mathbf{0 . 0 1 5 4} \\
(0.0456)\end{array}$ & $\begin{array}{l}\mathbf{- 0 . 0 1 4 9} \\
(0.0459)\end{array}$ & $\begin{array}{l}\mathbf{0 . 0 8 3 4} \\
(0.108)\end{array}$ & $\begin{array}{l}\mathbf{- 0 . 0 3 0 5} \\
(0.0638)\end{array}$ & $\begin{array}{l}\mathbf{- 0 . 0 6 7 2} \\
(0.0642)\end{array}$ \\
\hline $\begin{array}{l}\text { Log-transformed population } \\
\text { density (from zip) }\end{array}$ & $\begin{array}{r}\mathbf{0 . 1 3 6} \\
(0.133) \\
\end{array}$ & $\begin{array}{l}\mathbf{- 0 . 0 3 9 0} \\
(0.107) \\
\end{array}$ & $\begin{array}{l}\mathbf{- 0 . 0 3 2 5} \\
(0.109) \\
\end{array}$ & $\begin{array}{r}\mathbf{0 . 1 3 5} \\
(0.177) \\
\end{array}$ & $\begin{array}{l}\mathbf{- 0 . 1 7 4} \\
(0.154) \\
\end{array}$ & $\begin{array}{l}\mathbf{- 0 . 0 7 4 1} \\
(0.156) \\
\end{array}$ \\
\hline
\end{tabular}

Standard errors in parenthe

*** $\mathrm{p}<0.001,{ }^{* *} \mathrm{p}<0.01, * \mathrm{p}<0.05$

Table S3. Individual difference variables as moderators of treatment effects. Here we explore the extent to which our individual difference variables moderate our treatment effects. We report results from regressions predicting prevention intentions as a function of our individual difference variables, relevant condition contrasts, and their interactions, among all subjects (Columns 1-3) and subjects for whom we measured our dependent variables first (Columns 4-6). For each individual difference variable (in a series of separate regression models), shown is the interaction with (i) the overall treatment effect relative to control in Study 1 (Columns 1 and 4), and (ii) effects of the Public treatment relative to each of our other treatments, across the treatment conditions of Studies 1 and 2 (Columns 2-3 and 5-6). All coefficients are standardized coefficients, and standard errors are reported below each coefficient in parentheses. Before conducting these analyses, we (i) computed a "college degree" dummy from our measure of education, (ii) computed CRT scores (as the number of questions correct out of a possible three), 
and (iii) natural log-transformed our measure of population density. For our analyses of the overall treatment effect in Study 1, among all subjects $n=988$ for all variables except population density, for which $n=954$, and among subjects for whom we measured our dependent variables first, $n=506$ for all variables except population density, for which $n=487$. For our analyses of the Public treatment effects across Studies 1 and 2, among all subjects $n=$ 1930 for all variables except population density, for which $n=1845$, and among subjects for whom we measured our dependent variables first, $n=981$ for all variables except population density, for which $n=935$.

Table S3 reveals that we find no compelling evidence for moderation of our treatment effects. We find no significant moderation in our analyses of subjects for whom we measured our dependent variables first. In our analyses of all subjects, we also find no significant moderation of the overall treatment effect or the comparison of the Public vs. Personal treatments.

We do, however, find three significant moderators of the comparison of the Public vs. Personal+Public treatments. Specifically, as compared to Personal+Public, we observe relatively larger effects of the Public treatment among individuals who report higher subjective health, higher income, and stronger identification with the Democratic party. However, we note that for two of the significant interactions, conceptually related variables showed null effects (specifically, subjective health is conceptually related to pre-existing health conditions, and identification with the Democratic party is related to our other two political ideology variables). Furthermore, because our moderation analyses are only exploratory and we do not take them as strong evidence of any claims, Table S3 does not report q-values; however, it of course reports many tests, creating a multiple comparisons problem.

Thus, we ultimately do not see Table S3 as providing compelling evidence for moderation (without replication).

\subsection{Perceived public and personal threat of coronavirus across conditions in Studies 1-3}

Next, we discuss the perceived public and personal threat of coronavirus across conditions in Studies 1-3. We note that we analyze Studies 1 and 2 separately, because we measured these threat variables via slightly different wording in Studies 1 vs. 2; Studies 3a-d used the Study 2 wording. We also note that in Studies 1-2, due to a programming error, our personal vs. public threat variables differed in the order in which the two questions for each construct were presented; this error was corrected in Studies 3a-d. See Section 6 for more detail on the measurement of threat variables across studies.

As noted in the main text, across both Studies 1-2 and Studies 3a-d, neither of our threat variables differed significantly across conditions. In Table S4, we report descriptive statistics for each threat variable across conditions, both for our earlier studies (with separate results for Study 1 and Study 2) and later studies (with separate results for Studies 3a-d and Study 3d). Table S4 also allows interested readers to compare perceived threat levels earlier vs. later in the pandemic. 
Earlier studies

\begin{tabular}{|c|c|c|c|c|}
\hline & \multicolumn{2}{|c|}{ Study $1(n=988)$} & \multicolumn{2}{|c|}{ Study $2(n=1188)$} \\
\hline & Personal threat & Public threat & Personal threat & Public threat \\
\hline Control & $54.54[50.79,58.29]$ & $67.87[64.79,70.95]$ & & \\
\hline Personal Treatment & $58.82[55.21,62.43]$ & $70.57[67.55,73.59]$ & $57.9[55.14,60.66]$ & $70.4[68.2,72.6]$ \\
\hline Public Treatment & $54.75[51.19,58.31]$ & $71.1[68.17,74.02]$ & $57.15[54.24,60.05]$ & $69.56[67.25,71.86]$ \\
\hline \multirow[t]{4}{*}{ Personal + Public Treatment } & $55.65[51.91,59.39]$ & $71.31[68.52,74.11]$ & $57.57[54.65,60.49]$ & $70.44[68.23,72.66]$ \\
\hline & \multicolumn{4}{|c|}{ Later studies } \\
\hline & \multicolumn{2}{|c|}{ Studies 3a-d $(n=3985)$} & \multicolumn{2}{|c|}{ Study 3d $(n=1773)$} \\
\hline & Personal threat & Public threat & Personal threat & Public threat \\
\hline Personal Treatment & $60.59[59.07,62.12]$ & $68.99[67.71,70.27]$ & $57.21[54.85,59.58]$ & $66.39[64.36,68.43]$ \\
\hline Public Treatment & $60.93[59.39,62.47]$ & $70.58[69.33,71.83]$ & $56.67[54.28,59.06]$ & $67.11[65.07,69.15]$ \\
\hline Personal + Public Treatment & $60.5[59.02,61.99]$ & $69.23[67.98,70.47]$ & $57.17[54.95,59.39]$ & $67.26[65.34,69.19]$ \\
\hline
\end{tabular}

Table S4. Threat variables by study and condition. We report mean values (with $95 \%$ CIs) for each threat variable by study and condition.

In statistical analyses, we find no evidence that our threat variables differed across conditions. First, looking to Studies 1-2, for each threat variable we (i) compared each treatment to the control in Study 1, and (ii) compared each pair of treatments both within Study 1 and within Study 2. Next, looking to Study 3, for each threat variable we compared each pair of treatments, controlling for composite Time 1 prevention intentions, both within Studies 3a-d and within Study 3d. We found no significant results (all ps $>.05$ ), suggesting that our threat variables do not explain our treatment effects; thus, we do not report mediation analyses.

\subsection{Moderation by subjective health in Studies 1-2}

Next, we discuss moderation by subjective health across Studies 1-2. As noted in the main text, in Study 1, we found some evidence that individuals reporting greater subjective health showed relatively larger effects of the Public treatment on prevention intentions. This result makes theoretical sense: healthy individuals are at lower risk for coronavirus, and thus should be less likely to see prevention behaviors as self-interested and more likely to treat them like a public good. Thus, in our Study 2 pre-registration, we planned for our primary analyses to focus specifically on healthier individuals (defined as individuals reporting subjective health above the Study 1 median). However, evidence for an interaction between health and our Public treatment effects was weaker in Study 2 than in Study 1. Thus, despite the fact that moderation by subjective health makes theoretical sense, we did not feel confident focusing on health in our primary analyses, and instead chose to report main effects among all subjects. And our preregistrations for and analyses of Study 3 also focus on main effects among all subjects.

In Table S5, however, we report detailed analyses of subjective health in Studies 1-2. Our objective in doing so is to provide transparency with respect to our pre-registered plan to focus on health in Study 2. Thus, because our pre-registrations only planned analyses of all subjects, for brevity in this section we do not additionally report analyses of subjects for whom we measured our dependent variables first.

Specifically, Table S5 reports the effects of the Public treatment on prevention intentions, relative to our other treatments, as a function of subjective health. We conduct separate analyses of the treatment conditions of Study 1 (Column 1), Study 2 (Column 2), and Studies 1 and 2 combined (Column 3). In each analysis, we compare the Public treatment to the Personal treatment (top rows), and to the Personal+Public treatment (bottom rows). For each comparison, 
we report (i) the relative effect of the Public treatment, separately among healthier and less healthy subjects, and (ii) the interaction between (continuous) health and the Public (vs. other) treatment.
Study 1
Study 2
Studies 1 and 2
(Healthier $n=375$,
(Healthier $n=560$,
(Healthier $n=935$,
Less healthy $n=367$ )
Less healthy $n=628$ )
Less healthy $n=995$ )

\begin{tabular}{|c|c|c|c|}
\hline Public vs. Personal & & & \\
\hline Healthier & $\begin{aligned} \mathrm{b}=4.58, \mathrm{t} & =\mathbf{2 . 3 4 ,} \mathrm{d}=. \mathbf{2 9} \\
\mathrm{p} & =.020\end{aligned}$ & $\begin{array}{c}\mathbf{b}=\mathbf{2 . 5 5 ,} \mathbf{t}=\mathbf{1 . 7 5 ,} \mathbf{d}=\mathbf{. 1 8} \\
\mathrm{p}=.080\end{array}$ & $\begin{aligned} \mathbf{b}=\mathbf{3 . 3 5}, \mathbf{t} & =\mathbf{2 . 8 6 ,} \mathbf{d}=\mathbf{. 2 2}, \\
\mathrm{p} & =.004\end{aligned}$ \\
\hline Less healthy & $\begin{aligned} \mathbf{b}=\mathbf{0 . 7 2 ,} \mathbf{t} & =\mathbf{0 . 3 3}, \mathbf{d}=\mathbf{. 0 4} \\
\mathrm{p} & =.739\end{aligned}$ & $\begin{aligned} \mathbf{b}=\mathbf{2 . 2 5 ,} \mathbf{t} & =\mathbf{1 . 4 1 ,} \mathbf{d}=\mathbf{. 1 4} \\
\mathrm{p} & =.159\end{aligned}$ & $\begin{aligned} \mathbf{b}=\mathbf{1 . 6 9 ,} & \mathbf{t}=\mathbf{1 . 3 1 ,} \mathbf{d}=\mathbf{. 1 0}, \\
\mathrm{p} & =.189\end{aligned}$ \\
\hline $\begin{array}{c}\text { Interaction } \\
\text { (with continuous health) }\end{array}$ & $\begin{array}{c}\mathbf{b}=\mathbf{1 . 7 8}, \mathbf{t}=\mathbf{1 . 2 6} \\
\mathrm{p}=.208\end{array}$ & $\begin{array}{c}\mathbf{b}=\mathbf{1 . 3 3}, \mathbf{t}=\mathbf{1 . 2 5} \\
\mathrm{p}=.212\end{array}$ & $\begin{array}{c}\mathbf{b}=\mathbf{1 . 5 1}, \mathbf{t}=\mathbf{1 . 7 7} \\
\mathrm{p}=.076\end{array}$ \\
\hline \multicolumn{4}{|l|}{ Public vs. Personal + Public } \\
\hline Healthier & $\begin{aligned} \mathbf{b}=\mathbf{6 . 0 7 ,} & \mathbf{t}=\mathbf{3 . 0 2}, \mathbf{d}=\mathbf{. 3 8} \\
\mathrm{p} & =.003\end{aligned}$ & $\begin{aligned} \mathbf{b}=\mathbf{1 . 5 2}, \mathbf{t} & =\mathbf{1 . 0 3}, \mathbf{d}=\mathbf{. 1 1} \\
\mathrm{p} & =.304\end{aligned}$ & $\begin{aligned} \mathbf{b}=\mathbf{3 . 3 2}, \mathbf{t} & =\mathbf{2 . 7 7 ,} \mathbf{d}=\mathbf{. 2 2}, \\
\mathrm{p} & =.006\end{aligned}$ \\
\hline Less healthy & $\begin{array}{c}\mathbf{b}=\mathbf{- 1 . 9 5 ,} \mathbf{t}=\mathbf{- 0 . 9 1 ,} \mathbf{d}=\mathbf{- . 1 2} \\
\mathrm{p}=.361\end{array}$ & $\begin{aligned} \mathbf{b}=\mathbf{0 . 2 3 ,} \mathbf{t} & =\mathbf{0 . 1 5}, \mathbf{d}=\mathbf{. 0 1}, \\
\mathbf{p} & =.883\end{aligned}$ & $\begin{array}{c}b=-.58, t=-.45, d=-.04, \\
p=.651\end{array}$ \\
\hline $\begin{array}{c}\text { Interaction } \\
\text { (with continuous health) }\end{array}$ & $\begin{array}{c}b=\mathbf{3 . 3 9}, t=\mathbf{2 . 4 4} \\
\mathrm{p}=.015\end{array}$ & $\begin{array}{c}\mathbf{b}=\mathbf{1 . 1 2}, \mathbf{t}=\mathbf{1 . 0 9} \\
\mathrm{p}=.278\end{array}$ & $\begin{array}{c}\mathbf{b}=\mathbf{2 . 0 0}, \mathbf{t}=\mathbf{2 . 4 1} \\
\mathrm{p}=.016\end{array}$ \\
\hline
\end{tabular}

Table S5. Effects of the Public treatment as a function of subjective health. Here we report effects of the Public treatment on prevention intentions, both relative to the Personal treatment and the Personal+Public treatment. For each treatment contrast, we report the effect of the Public (vs. other) treatment among healthier and less healthy individuals, as well as the interaction between our (continuous) subjective health measure and the Public (vs. other) treatment. We report these analyses of all subjects across the treatment conditions of (i) Study 1 (Column 1) ( $n=742)$, (ii) Study 2 (Column 2) $(n=1188)$, and (ii) Studies 1 and 2 combined (Column 3) $(n=1930)$.

Table S5 illustrates that (i) we found some evidence for an interaction between health and the effects of our Public treatment in Study 1, but (ii) we did not find meaningful support for this pattern in Study 2. Thus, our results do not provide robust support for the proposal that the Public treatment is especially effective among healthier individuals.

Table S5 also reveals that in analyses of only healthier individuals (i.e., the population that we planned to focus on in our Study 2 pre-registration), we continue to find evidence that the Public treatment was more effective than the Personal treatment, and no less effective than the Personal+Public treatment. Furthermore, the difference between the Public and Personal treatments observed in the pooled analysis of Studies 1 and 2 holds when accounting for multiple comparisons ( $p=.004, q_{c}=.026, q_{s}=.024$ ), and when accounting for "peeking" (to maintain an actual type-I error rate of .05, it is necessary to evaluate statistical significance using an alpha threshold ranging from a "best-case scenario" of .049999 to a "worst-case scenario" of .0283).

\subsection{Did the relative effectiveness of treatments depend on Time 1 prevention intentions in Study 3 ?}

Here, we discuss the question of whether the relative effectiveness of our treatments in Study 3 varied as a function of Time 1 prevention intentions. To address this question, we shape our data to long format (with one observation per prevention intention item per subject) and use robust standard errors clustered on subject. Then, we conduct two sets of analyses. First, we 
predict Time 2 prevention intentions as a function of treatment dummies, Time 1 prevention intentions, and their interactions. In the top rows of Table S6, we report the interaction for each pairwise comparison between treatments.

Second, we predict Time 2 prevention intentions as a function of treatment dummies and Time 1 prevention intentions, among observations for which the Time 1 prevention intention value is relatively lower (specifically, less than 80 on our 100-point scale; this pre-registered threshold is close to the median Time 1 prevention intention value, which is 79 both in Studies 3a-d and in Study 3d). In the bottom rows of Table S6, we compare each pair of treatments for this set of observations.

As illustrated by Table S6, across both analyses, we find no compelling evidence that the relative effectiveness of our treatments varied as a function of Time 1 prevention intentions.

$$
\text { Studies 3a-d }(n=3985) \quad \text { Study 3d }(n=1773)
$$

\begin{tabular}{|c|c|c|}
\hline & \multicolumn{2}{|c|}{ Interaction between Time 1 intentions and pairwise comparison } \\
\hline $\begin{array}{l}\text { Public vs. } \\
\text { Personal }\end{array}$ & $\begin{array}{c}\mathbf{b}=\mathbf{- 0 . 0 1}, \mathbf{t}=\mathbf{- 0 . 5 1} \\
\mathrm{p}=.607, \mathrm{q}_{\mathrm{c}}=.939\end{array}$ & $\begin{array}{c}\mathbf{b}=\mathbf{- 0 . 0 2}, \mathbf{t}=\mathbf{- 0 . 9 7} \\
\mathrm{p}=.331, \mathrm{q}_{\mathrm{c}}=.701\end{array}$ \\
\hline $\begin{array}{c}\text { Public vs. } \\
\text { Personal+Public }\end{array}$ & $\begin{array}{c}\mathbf{b}=\mathbf{- 0 . 0 2}, \mathbf{t}=\mathbf{- 1 . 1 6} \\
\mathrm{p}=.244, \mathrm{q}_{\mathrm{c}}=.568\end{array}$ & $\begin{array}{c}\mathbf{b}=\mathbf{0 . 0 0}, \mathbf{t}=\mathbf{0 . 0 7} \\
\mathrm{p}=.943, \mathrm{q}_{\mathrm{c}}=1.000\end{array}$ \\
\hline \multirow[t]{2}{*}{$\begin{array}{c}\text { Pesonal vs. } \\
\text { Personal+Public }\end{array}$} & $\begin{array}{c}\mathbf{b}=\mathbf{- 0 . 0 1}, \mathbf{t}=\mathbf{- 0 . 6 2} \\
\mathrm{p}=.537, \mathrm{q}_{\mathrm{c}}=.901\end{array}$ & $\begin{array}{l}\mathbf{b}=\mathbf{0 . 0 2}, \mathbf{t}=\mathbf{1 . 0 6} \\
\mathrm{p}=.291, \mathrm{q}_{\mathrm{c}}=.643\end{array}$ \\
\hline & \multicolumn{2}{|c|}{ Pairwise comparison if Time 1 intentions $<80$} \\
\hline $\begin{array}{l}\text { Public vs. } \\
\text { Personal }\end{array}$ & $\begin{array}{c}\mathbf{b}=\mathbf{0 . 4 1}, \mathbf{t}=\mathbf{0 . 7 8}, \mathbf{d}=\mathbf{0 . 0 1} \\
\mathrm{p}=.435, \mathrm{q}_{\mathrm{c}}=.820\end{array}$ & $\begin{array}{c}\mathrm{b}=\mathbf{1 . 0 4}, \mathbf{t}=\mathbf{1 . 3 6}, \mathbf{d}=\mathbf{0 . 0 4} \\
\mathrm{p}=.176, \mathrm{q}_{\mathrm{c}}=.440\end{array}$ \\
\hline $\begin{array}{c}\text { Public vs. } \\
\text { Personal+Public } \\
\end{array}$ & $\begin{array}{c}\mathbf{b}=\mathbf{1 . 0 1}, \mathbf{t}=\mathbf{2 . 0 1}, \mathbf{d}=\mathbf{0 . 0 4} \\
\mathrm{p}=.045, \mathrm{q}_{\mathrm{c}}=.129\end{array}$ & $\begin{array}{c}\mathbf{b}=\mathbf{0 . 5 8}, \mathbf{t}=\mathbf{0 . 7 6}, \mathbf{d}=\mathbf{0 . 0 2} \\
\mathrm{p}=.448, \mathrm{q}_{\mathrm{c}}=.832\end{array}$ \\
\hline $\begin{array}{c}\text { Pesonal vs. } \\
\text { Personal+Public }\end{array}$ & $\begin{array}{c}\mathbf{b}=\mathbf{0 . 6 0}, \mathbf{t}=\mathbf{1 . 2 0}, \mathbf{d}=\mathbf{0 . 0 2} \\
\mathrm{p}=.232, \mathrm{q}_{\mathrm{c}}=.547\end{array}$ & $\begin{array}{c}\mathbf{b}=\mathbf{- 0 . 4 6 ,} \mathbf{t}=\mathbf{- 0 . 6 1}, \mathbf{d}=\mathbf{- 0 . 0 2} \\
\mathrm{p}=.543, \mathrm{q}_{\mathrm{c}}=.905\end{array}$ \\
\hline
\end{tabular}

Table S6. Investigating the relative effectiveness of treatments as a function of Time 1 prevention intentions. For each pairwise comparison, we report (i) the interaction between the relative effect of the treatment listed first and Time 1 prevention intentions on Time 2 prevention intentions (top rows), and (ii) the relative effect of the treatment listed first on Time 2 prevention intentions, controlling for Time 1 prevention intentions, among observations where the Time 1 prevention intention value is less than 80 (bottom rows). We report results both across Studies $3 a-d$, and in Study $3 d$ only. We note that because our data are in long format, the standard deviation across observations is higher and Cohen's $d$ values are lower, preventing a straightforward comparison to Cohen's $d$ values from our wide format analyses.

\subsection{Analyses of "English check" in Studies 1-2 and attention checks in Study 3}

In our Study 1-2 pre-registrations, we planned to conduct secondary analyses excluding subjects who appear not to speak English, on the basis of incorrect answers to a simple analogy question or incoherent responses to a simple free-response question. We coded answers to the simple analogy question (in a way that was blind to condition) for correct or near-correct answers (i.e., correct answers with typos/misspellings); across both studies (and all subjects), $6.99 \%$ of responses were incorrect. A visual scan of our data revealed that most subjects who 
answered the analogy question incorrectly provided incoherent and/or irrelevant responses to the free-response question, while the vast majority of subjects who answered the analogy question correctly provided coherent and relevant answers. On this basis, we repeated our analyses excluding subjects who incorrectly answered the analogy question. We found that our results were unchanged qualitatively, but most patterns became a bit stronger. For brevity, we do not report these analyses; however, our "English check" data are available to interested readers.

In Study 3, we did not include this "English check"; however, in Studies 3a-c we instead asked subjects two simple attention checks (in which the question text instructed attentive subjects to select specific answer choices) and pre-registered secondary analyses investigating how our results vary for differing levels of attentiveness. We find no evidence that our key results vary significantly as a function of attentiveness; again, for brevity, we do not report these analyses but do make our attention check items available to interested readers.

\subsection{Alternative choices about public and personal threat items in Study 4}

In Study 4, we focused on certain specific items from Pennycook et al. 2020 that we felt mapped most closely onto our constructs of public versus personal threat (and our measures of these constructs from Studies 1-3). Here we demonstrate that the results are robust to different decisions about which items to use.

We begin by listing all items collected. Subjects rated their agreement $(1=$ Strongly disagree, $2=$ Disagree, $3=$ Somewhat disagree, $4=$ Neither agree nor disagree, $5=$ Somewhat agree, $6=$ Agree, $7=$ Strongly agree) with the following statements about COVID-19 risk:

1. The coronavirus poses a major threat to the public.

2. The coronavirus spread much faster than anyone anticipated.

3. People are not taking the coronavirus as seriously as they ought to.

4. There should be mass testing for the coronavirus.

5. The coronavirus is not as dangerous as people think. (R)

6. I think the situation with the coronavirus is overblown. (R)

7. Most people are not at risk of contracting the coronavirus. (R)

8. Very few people in the country are likely to actually get sick from the coronavirus. (R)

as well as these three items explicitly about themselves personally:

9. Because of my location, profession, and/or lifestyle, I am personally at a high risk of contracting the coronavirus.

10. Because of my age and/or pre-existing conditions, I am likely to have serious symptoms if I were to contract the coronavirus.

11. Because of my age and/or pre-existing conditions, I am likely to need hospitalization if I were to contract the coronavirus.

Our main text analysis uses item 1 as a measure of public threat and items 10 and 11 as a measure of personal threat. Table $\mathrm{S} 7$ shows the results using a variety of different combinations of items, revealing that our findings are robust to choices in measure construction. Below, we briefly discuss the logic behind (i) the set of combinations we chose to include in Table S7 and (ii) our choice of measures for our main text analysis. 
In most analyses in Table S7, we include public threat measures constructed from some combination of items 1,7 , and 8 , because these are the items that explicitly reference the public/population at large. We also saw items 1 and 8 as especially relevant because, like our measures from Studies 1-3, they focus on threat to the public and the consequences (rather than likelihood) of contracting coronavirus. And we chose to specifically use item 1 in our main text analyses because of its high face validity and because, like our measures from Studies 1-3, it is straightforwardly worded (i.e., not reverse coded).

Turning to personal threat, items 9-11 all explicitly reference the individual. We also saw items 10 and 11 as especially relevant because, like our measures from Studies 1-3, they focus on the consequences (rather than likelihood) of contracting coronavirus. We thus chose to use them in our primary analysis as our measure of personal threat. 


\begin{tabular}{|c|c|c|c|c|c|c|c|c|c|}
\hline \multirow{2}{*}{ Personal threat } & \multicolumn{3}{|c|}{ Public $=1$ v Personal $=10,11$} & \multicolumn{3}{|c|}{ Public $=1,8$ v Personal $=10,11$} & \multicolumn{3}{|c|}{ Public $=1,7,8$ v Personal $=10,11$} \\
\hline & $\begin{array}{c}\mathbf{0 . 2 8 2} * * * \\
(0.0351)\end{array}$ & $0.191 * * *$ & $0.179^{* * * *}$ & $0.282 * * *$ & $0.197^{* * *}$ & $0.187 * * *$ & $0.282^{* * *}$ & $0.209^{* * * *}$ & $0.193^{* * * *}$ \\
\hline & & & & & & & $* * *$ & & \\
\hline $\begin{array}{c}\text { Public vs. } \\
\text { Personal }\end{array}$ & $\begin{array}{c}t(745)=3.24 \\
p=0.001\end{array}$ & $\begin{array}{c}\mathrm{F}(1,745)=11.56 \\
\mathrm{p}<0.001\end{array}$ & $\begin{array}{c}\mathrm{F}(1,709)=8.58 \\
\mathrm{p}=0.004\end{array}$ & $\begin{array}{c}\mathrm{t}(745)=2.81 \\
\mathrm{p}=0.005\end{array}$ & $\begin{array}{c}\mathrm{F}(1,745)=8.66 \\
\mathrm{p}=0.003\end{array}$ & $\begin{array}{c}\mathrm{F}(1,709)=6.40 \\
\mathrm{p}=0.012\end{array}$ & $\begin{array}{c}\mathrm{t}(745)=2.23 \\
\mathrm{p}=0.026\end{array}$ & $\begin{array}{c}\mathrm{F}(1,745)=5.49 \\
\mathrm{p}=0.019\end{array}$ & $\begin{array}{c}\mathrm{F}(1,709)=4.36 \\
\mathrm{p}=0.037\end{array}$ \\
\hline
\end{tabular}

\begin{tabular}{|c|c|c|c|c|c|c|c|c|c|}
\hline \multirow[b]{2}{*}{ Personal threat } & \multicolumn{3}{|c|}{ Public $=1$ v Personal $=9,10,11$} & \multicolumn{3}{|c|}{ Public $=1,8$ v Personal $=9,10,11$} & \multicolumn{3}{|c|}{ Public $=1,7,8$ v Personal $=9,10,11$} \\
\hline & & & & $\begin{array}{l}\mathbf{3 0 3} * * * \\
0.0349)\end{array}$ & & & $\begin{array}{c}\mathbf{0 . 3 0 3} * * * \\
(0.0349)\end{array}$ & $\begin{array}{c}\mathbf{0 . 2 1 8 * * *} \\
(0.0342)\end{array}$ & \\
\hline Public t & $\begin{array}{c}\mathbf{0 . 4 1 8}^{* * *} \\
(0.0333)\end{array}$ & $\begin{array}{c}\mathbf{0 . 3 6 1 * * *} \\
(0.0339)\end{array}$ & $\begin{array}{c}\mathbf{0 . 3 4 6 * * *} \\
(0.0356)\end{array}$ & $\begin{array}{c}\mathbf{0 . 4 0 1} * * * \\
(0.0335)\end{array}$ & $\begin{array}{c}\mathbf{0 . 3 4 2} * * * \\
(0.0343)\end{array}$ & $\begin{array}{r}\mathbf{0 . 3 3 2} \\
(0.03\end{array}$ & $\begin{array}{c}\mathbf{0 . 3 7 9 * * *} \\
(0.0339)\end{array}$ & $\begin{array}{c}\mathbf{0 . 3 2 1} * * * \\
(0.0342)\end{array}$ & $\begin{array}{r}\mathbf{0 . 3 1 3} * * * \\
(0.0374)\end{array}$ \\
\hline $\begin{array}{l}\text { Public vs. } \\
\text { Personal }\end{array}$ & $\begin{array}{c}\mathrm{t}(745)=2.84 \\
\mathrm{p}=0.005\end{array}$ & $\begin{array}{c}F(1,745)=8.77 \\
p=0.003\end{array}$ & $\begin{array}{c}\mathrm{F}(1,709)=7.14 \\
\mathrm{p}=0.008\end{array}$ & $\begin{array}{c}\mathrm{t}(745)=2.41 \\
\mathrm{p}=0.016\end{array}$ & $\begin{array}{c}\mathrm{F}(1,745)=6.29 \\
\mathrm{p}=0.012\end{array}$ & $\begin{array}{c}\mathrm{F}(1,709)=5.12 \\
\mathrm{p}=0.024\end{array}$ & $\begin{array}{c}f(745)=1.81 \\
p=0.07\end{array}$ & $\begin{array}{c}\mathrm{F}(1,745)=3.59 \\
\mathrm{p}=0.059\end{array}$ & $\begin{array}{c}\mathrm{F}(1,709)=3.21 \\
\mathrm{p}=0.074\end{array}$ \\
\hline
\end{tabular}

\begin{tabular}{c|ccc|}
\cline { 2 - 4 } & \multicolumn{3}{|c|}{ Public $=1-8$ v Personal $=9,10,11$} \\
\hline Personal threat & $\mathbf{0 . 4 8 6} * * *$ & $\mathbf{0 . 4 3 4} * * *$ & $\mathbf{0 . 4 4 6} * * *$ \\
Public threat & $(0.0320)$ & $(0.0332)$ & $(0.0370)$ \\
& $\mathbf{0 . 3 0 3 * * *}$ & $\mathbf{0 . 1 6 4} * * *$ & $\mathbf{0 . 1 3 8} * * *$ \\
Public vs. & $(0.0349)$ & $(0.0332)$ & $(0.0377)$ \\
Personal & $\mathrm{t}(745)=4.78$ & $\mathrm{~F}(1,745)=24.93$ & $\mathrm{~F}(1,709)=25.22$ \\
\hline
\end{tabular}

Standard errors in parentheses

$* * * \mathrm{p}<0.01, * * \mathrm{p}<0.05, * \mathrm{p}<0.1$

Table S7. Study 4's observation that public threat is a stronger predictor of prevention intentions is robust to choices in measure construction. Shown are the results from regressions predicting prevention intentions as a function of the composition thread variables collected in Study 4 using various different formulations of public versus personal threat. Each box heading reports the item(s) used to form the public and personal threat scales. We then show, in each box, results from (i) a set of separate regression models for each threat variable (left column within each box) and (ii) multiple regression models using both threat variables, both without (center column within each box) and with (right column within each box) controls for age, gender, education, race, income, and political party affiliation. All coefficients are standardized coefficients, with standard errors for each coefficient in parentheses. For each model, we also report results from a test comparing the public versus personal threat coefficient. 


\section{Discussion of pre-registered analysis plans}

We independently pre-registered Studies 1 (https://aspredicted.org/wk9xj.pdf), 2 (https://aspredicted.org/bw74n.pdf), 3a (https://aspredicted.org/6q2zt.pdf), 3b (https://aspredicted.org/nm9at.pdf), 3c (https://aspredicted.org/6qg6a.pdf), and 3d (https://aspredicted.org/pt8jg.pdf). We adhered closely to our pre-registered analysis plans, with a few exceptions. The substantive exceptions are noted in the main text where relevant, but here we provide a comprehensive list of all deviations. We also note that Studies 4 and 5, as well as our analyses in Studies 1-3 investigating the associations between our personal and public threat variables and prevention intentions, were not pre-registered. However, the fact that our personal and public threat analyses replicate across studies provides evidence against false positives.

\subsection{Studies 1-2}

First, in both of our Study 1 and 2 pre-registrations, we planned only to report results among all subjects, and not to explore the order in which we measured our dependent variables versus potential mediators. However, after completing both studies, we discovered an unexpected interaction between condition and order. Thus, to confirm the robustness of our results, for analyses of our dependent variables, we report results (i) among all subjects, and (ii) among subjects for whom we measured our dependent variables before measuring our potential mediators.

Second, in our Study 1 pre-registration, we planned to focus equally on both of our dependent variables (i.e., prevention intentions and social distancing intentions). However, as mentioned in main text, in Study 1 the prevention intentions variable produced stronger evidence for treatment effects and interesting differences between treatments, and thus in Study 2 we chose to focus on replicating these results. For this reason, we focus our paper on prevention intentions. Specifically, while we report primary analyses of social distancing intentions (i.e., treatment effects relative to control, and comparisons between treatment effects), we do not report analyses of the relationships between social distancing intentions and our individual difference variables or candidate mediators, or heterogeneity in treatment effects on intentions to avoid individual social behaviors.

Third, in our Study 1 pre-registration, we planned to compare all pairs of treatments to each other. However, given our pattern of results, when analyzing prevention intentions we chose to focus on the comparison of the Public treatment to each of the other two treatments, and thus do not compare the Personal treatment to the Public+Personal treatment. We pre-registered this plan before running Study 2.

Fourth, in our Study 1 pre-registration, we planned, as a secondary analysis, to explore treatment effects on intentions to engage in individual prevention behaviors, and to avoid individual social activities. Additionally, we noted that we were in particular concerned about ceiling effects, and thus would repeat our primary analyses looking only to the prevention behaviors and social activities for which baseline responses were the relatively lowest (i.e., furthest from ceiling). We did, in fact, explore individual prevention behaviors (see Figure S1), and in the main text we also report an analysis of the overall social distancing item included in our composite measure of prevention intentions. But because we did find treatment effects on prevention intentions (i.e., there was not a ceiling effect) and we found no significant heterogeneity across individual behaviors, we did not repeat our primary analyses looking only to behaviors furthest from ceiling. We note, however, that Figure S1 sorts individual behaviors 
by average baseline responses (i.e., distance from ceiling) for interested readers. (As noted above, we also did not explore intentions to avoid individual social activities, given our primary focus on our prevention intentions dependent variable).

Fifth, in our Study 2 pre-registration, we planned for our primary analyses to focus specifically on healthier individuals (defined as individuals reporting subjective health above the Study 1 median). As noted in the main text and the "moderation by subjective health" section of this SI, this decision reflected that, in Study 1, we found evidence suggesting that healthier individuals show relatively larger Public treatment effects. However, evidence for an interaction between health and our Public treatment effects was weaker in Study 2 than in Study 1. Thus, despite the fact that this interaction pattern makes theoretical sense, we did not feel confident focusing on it in our primary analyses, and instead chose to focus primarily on main effects among all subjects. We note, however, that as shown in the "moderation by subjective health" section of this document, analyses of healthy individuals also support our key findings from Studies 1-2.

Relatedly, in our Study 2 pre-registration, we also planned, as a secondary analyses, to (i) repeat our primary analyses among subjects reporting zero pre-existing health conditions, and (ii) test for interactions between pre-existing health conditions and the Public treatment. But because we chose not to focus extensively on moderation by health, we do not report these analyses.

Finally, our pre-registrations for Studies 1-2 did not plan to compute q-values to correct for multiple comparisons. We also only pre-registered each of our two studies individually and did not pre-register a plan to pool data from both studies.

\subsection{Study 3}

First, in our Study 3 pre-registrations, we planned to analyze our data in long format (with one observation per prevention intention item per subject). However, as described in the main text, for consistency with our approach from Studies 1-2, we instead primarily analyze data in wide format (computing composite prevention intentions across our 10 items). We do, however, analyze the data in long format as planned when investigating whether the relative effectiveness of treatments is influenced by Time 1 prevention intentions. We note that our conclusions from all analyses are qualitatively unchanged when analyzing in wide versus long format.

Second, in our Study 3 pre-registrations, we planned to explore moderation by our individual difference variables. However, given that we do not find any overall differences in the effectiveness of our treatments, for brevity we do not report moderation analyses.

Third, in our Study 3 pre-registrations, we simply planned to investigate absolute ratings of our two threat variables (i.e., the perceived public and personal threat of coronavirus) for the purpose of comparison to our earlier studies. However, we nonetheless investigate whether these variables showed the same patterns as in our earlier studies (both with respect to whether they were influenced by our manipulations, and their associations with prevention intentions).

Fourth, while each of our Study 3b-d pre-registrations planned to investigate the effectiveness of each of our treatments (by comparing Time 2 prevention intentions to Time 1 prevention intentions), we accidentally omitted this analysis from our Study 3a pre-registration. We nonetheless performed this analysis using data from Study 3a.

Finally, we only pre-registered each of Studies 3a-d individually and did not pre-register a plan to pool data across studies. 


\section{Experimental materials}

Here, we show the stimuli used in our studies. Additionally, all experimental materials are available at https://osf.io/sr4n9/.

\subsection{Written text from treatments}

In the main text, we report the sections of the written text used in our treatments that varied across treatments. Here, we report the sections that were constant across treatments.

Studies 1-2, before the section that varied across treatments:

Coronavirus disease 2019 (COVID-19) is a respiratory illness that can spread from person to person. The virus that causes COVID-19 is a novel coronavirus that was first identified during an investigation into an outbreak in Wuhan, China. Because COVID-19 is a novel virus, there is no immunity in the community yet. There is also no vaccine for COVID-19.

COVID-19 is currently spreading rapidly through the US. As of today, there are at least 1,701 confirmed cases, and this number is likely a major underestimate given that testing in the US has been extremely limited. The number of cases is growing exponentially. According to one projection by the Center for Disease Control (CDC), between 160 million and 214 million people in the U.S. could be infected over the course of the epidemic. As many as 200,000 to 1.7 million people could die. And, the calculations based on the CDC's scenarios suggested, 2.4 million to 21 million people in the U.S. could require hospitalization, potentially crushing the nation's medical system, which has only about 925,000 staffed hospital beds. Fewer than a tenth of those are for people who are critically ill.

COVID-19 is much worse than the ordinary flu. The flu has a death rate of around $0.1 \%$ of infections. Globally, about 3.4 percent of reported COVID-19 cases have died. Furthermore, experts think COVID-19 is more contagious than the ordinary flu. And people can spread COVID-19 before experiencing any symptoms.

Note that in Study 2, we updated the case count information to read "As of Sunday night, there are now over 3,000 confirmed cases".

Studies 1-2, after the section that varied across treatments:

It is recommended that you practice good personal hygiene (wash your hands, avoid shaking hands or hugging others, avoid touching your face, and cover your mouth when you cough or sneeze), stay home if you are even a little bit sick, practice social distancing (by staying home as much as possible and avoiding close contact with others), and prepare by purchasing food reserves, medication, and cleaning supplies.

Study 3, before the section that varied across treatments: 
Coronavirus disease 2019 (COVID-19) is spreading rapidly through the United States. As of Thursday night, there are now over 680,000 confirmed cases and over 35,000 deaths in the U.S. One factor that makes COVID-19 so difficult to contain is that people can spread the virus before experiencing any symptoms.

There is a long road ahead before life can return to normal. Most experts expect that a vaccine will not be ready for 12-18 months. And until a vaccine is ready, it will remain possible for new outbreaks to emerge.

Note that in Studies 3b-d, we updated the case and death count information to 800,000 and 45,000 (Study 3b), 850,000 and 47,000 (Study 3c), and 1,064,000 and 61,600 (Study 3d).

Study 3, after the section that varied across treatments:

Specifically, it is important to engage in social distancing by minimizing physical interactions, wearing a mask when outside the house, and staying at least $6 \mathrm{ft}$ away from others. It is also still important to practice good hygiene by washing hands frequently and trying not to touch your face. These actions are likely to remain important even after government "stay at home" orders end.

Furthermore, it may also be important for people to allow the government to access their health data and track their movements and the people who they are interacting with (i.e., "contract tracing") and/or regularly get tested for the virus. 


\subsection{Dependent variables}

Studies 1-2 and 4 measure of prevention intentions:

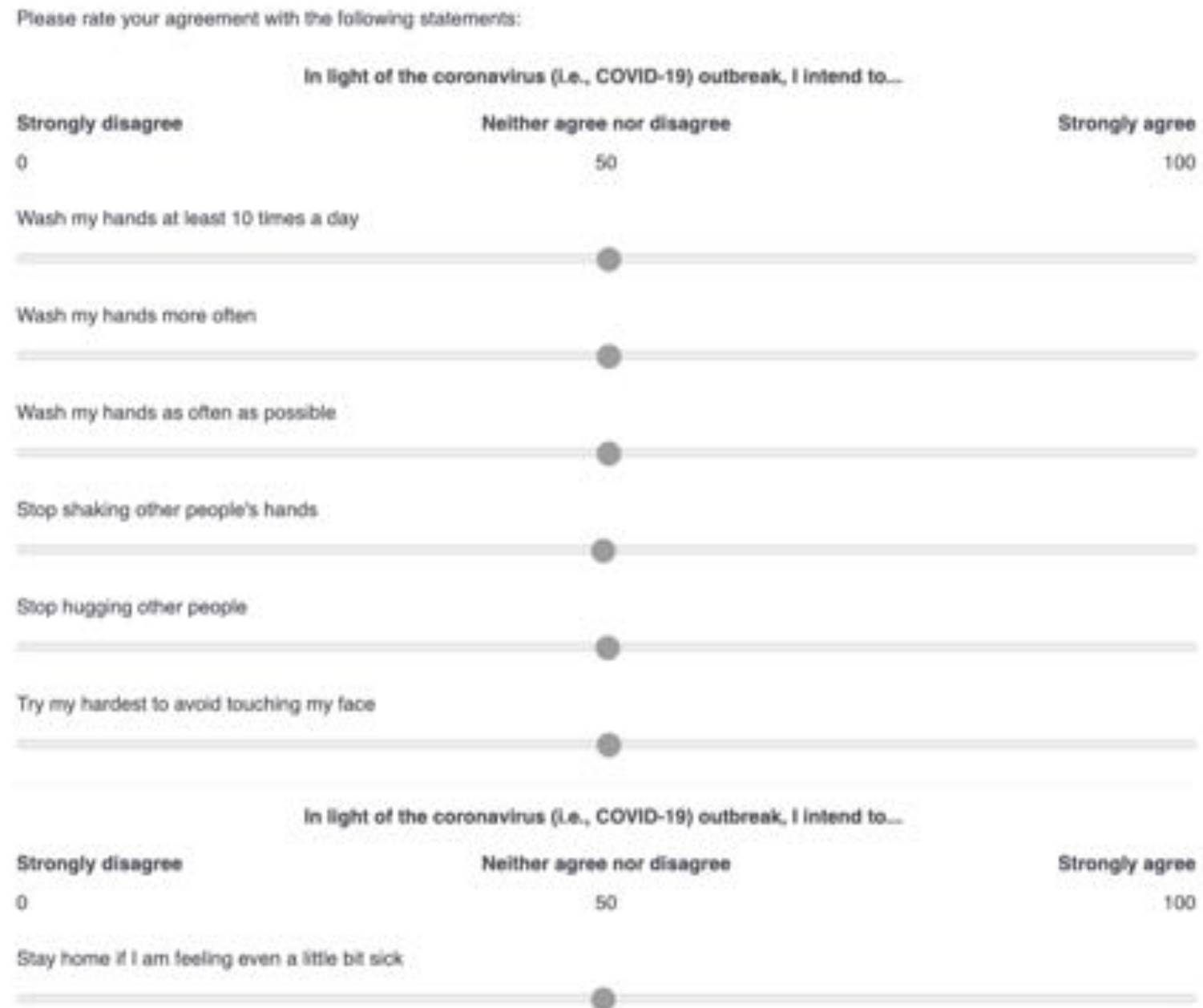

Try to stay home whenerer possble, even if I am not sick

Cover my mouth when I cough and snetexe

Purchase food teserves and medication

Stock up on cleaning supplies

Note that these items were presented in the fixed order shown here. 
Study 1 measure of social distancing intentions:

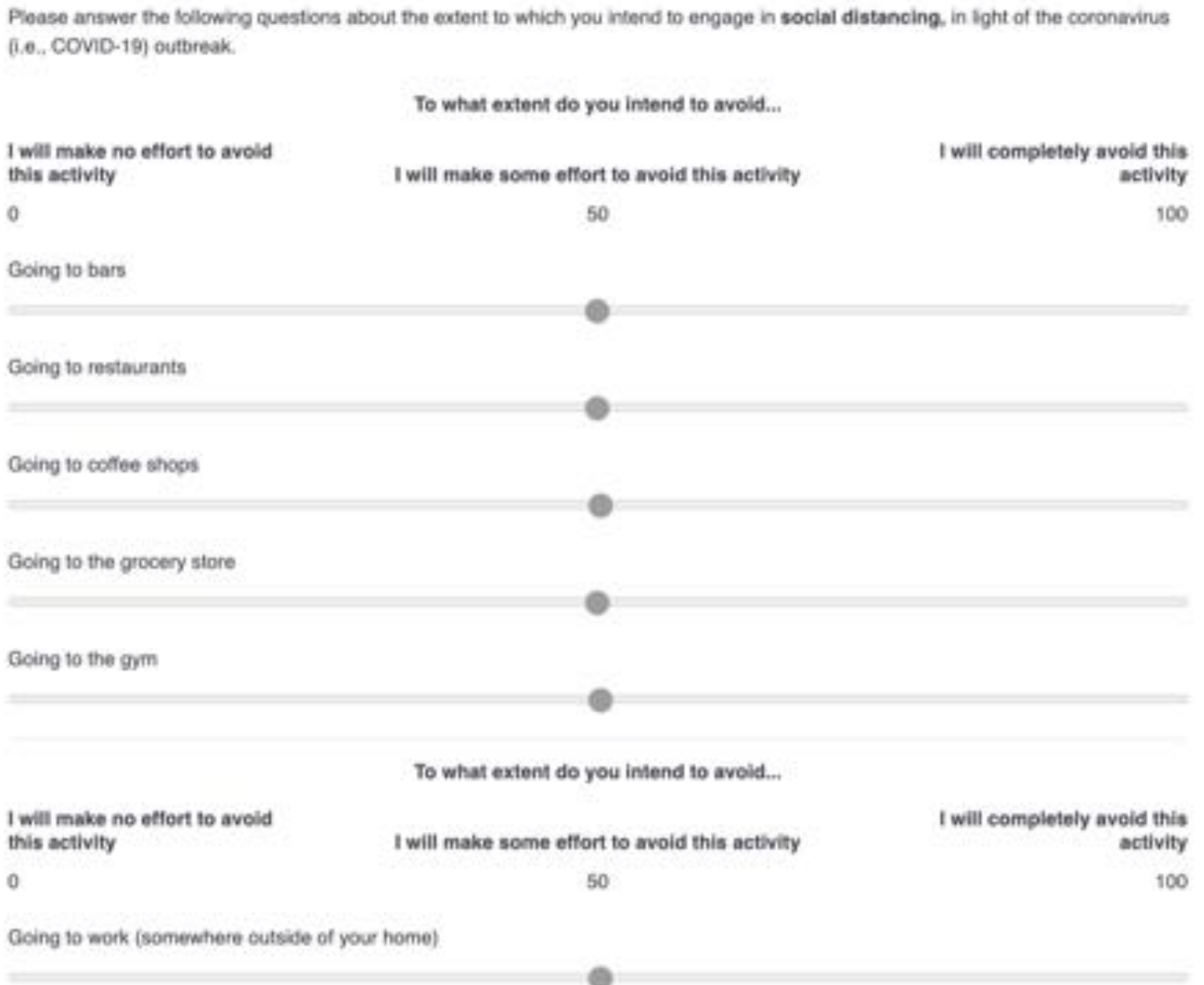

Ueing puble traneportation

Going to the airport and flying

Socializing in smal gatherings

Atlending large events or gatherings

Note that these items were presented in the fixed order shown here. 


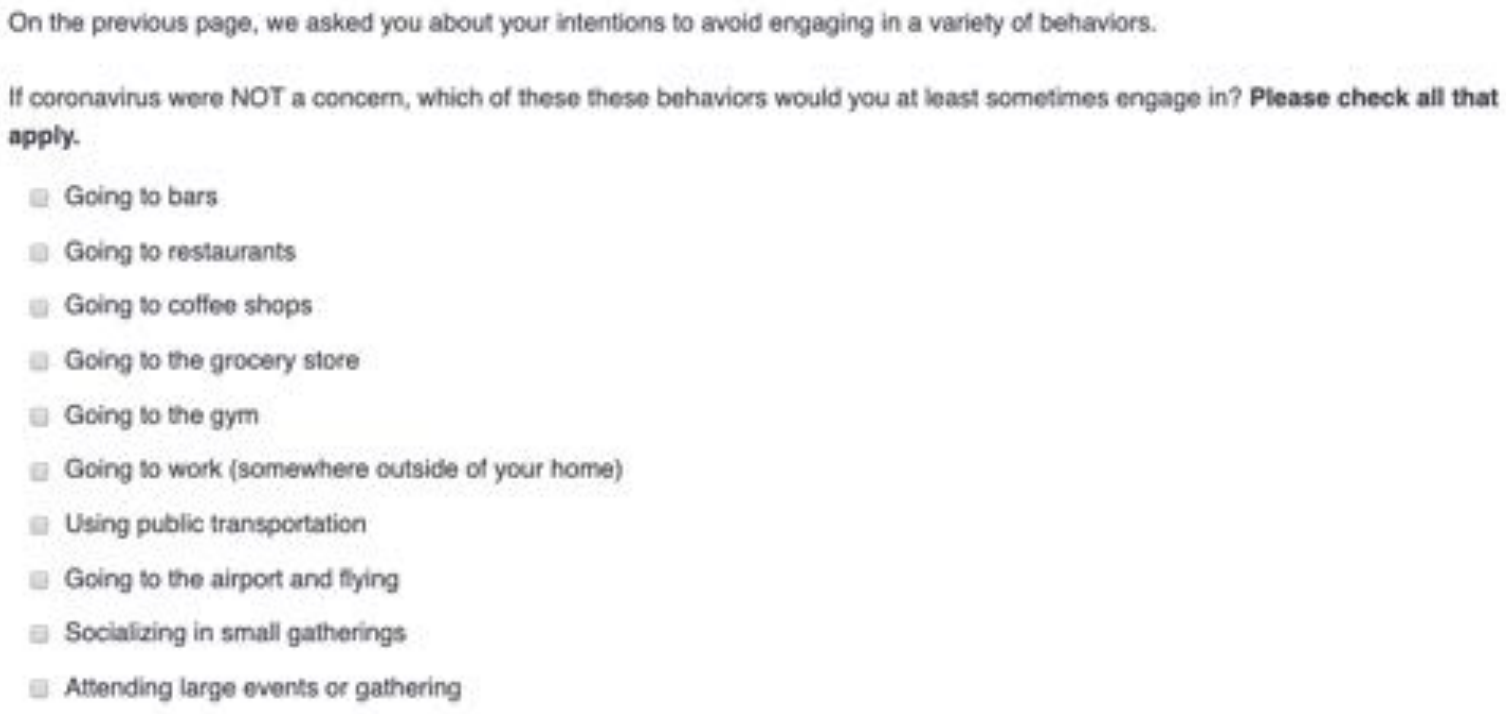

Note that these items were presented in the fixed order shown here. 
Study 3 measure of prevention intentions:

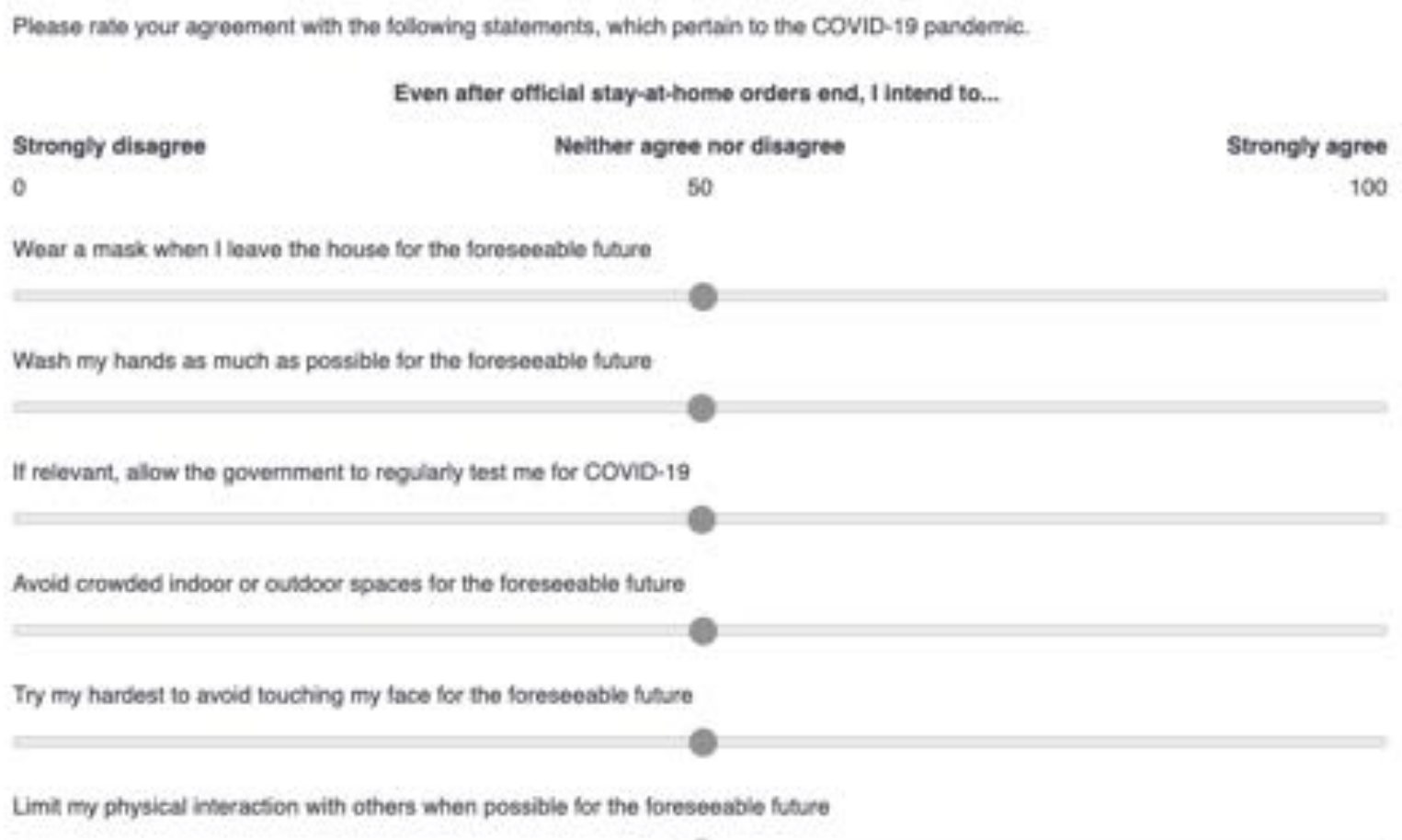

Relrain from visiting aryone who is sick at the hospital, even if they are a close tamily member and even if they are dying

Even if I think. I have contracled and cecovered thom COviD-19, remain vigilart unless I have a cerfirmed posifve lest (eatsar for the virus during infection, or antibodies after infection)

Completely avoid any unnecessary physical contact with others (e.g, hugging or handshakes) for the foreseenbie future

If relevant, alow the government to track my health data, movements, andjor the pecple I interact with

Note that these items were presented in random order, and this screenshot shows our Time 1 measure of prevention intentions. Our Time 2 measure was identical, except (i) the introductory statement at the top was replaced with "Please now rate your agreement with the following statements a second time", and (ii) the order was re-randomized. 


\subsection{Perceived public and personal threat of coronavirus}

Study 1 measure of perceived personal threat:

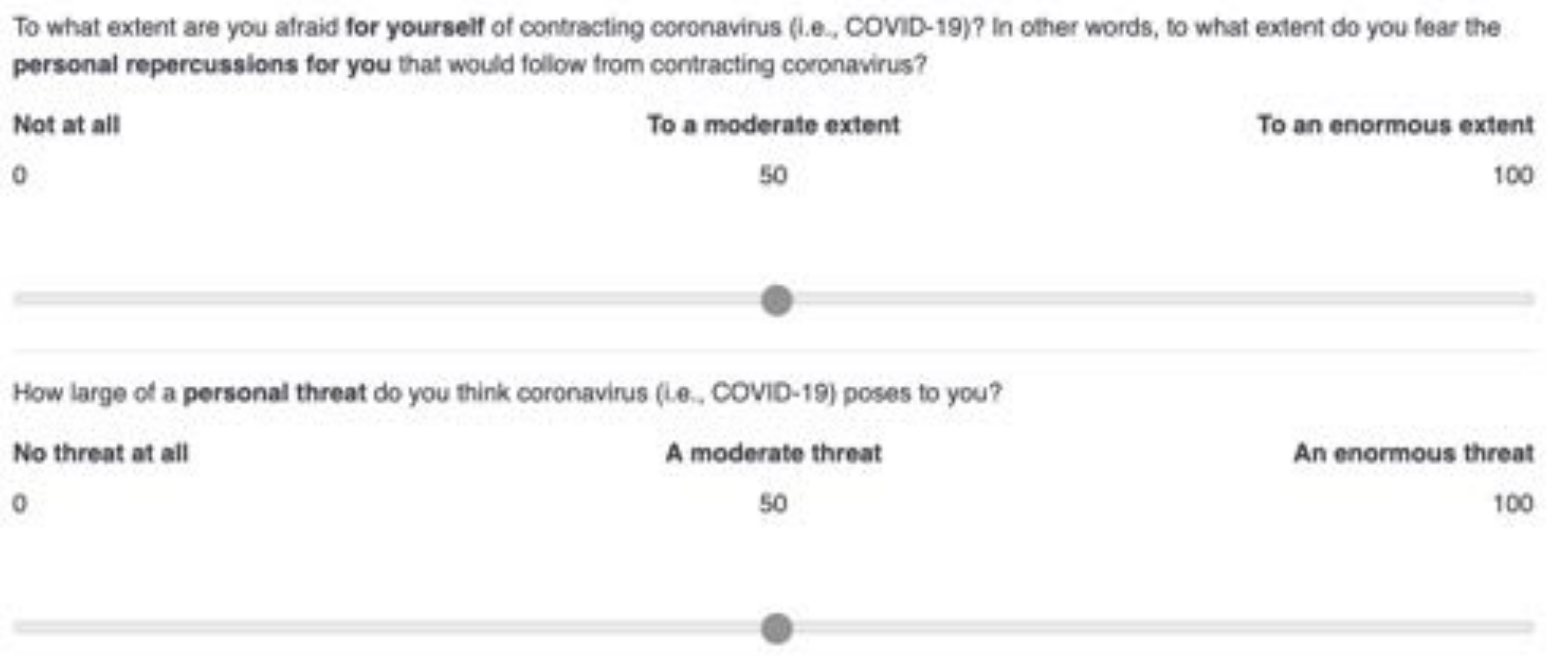

Study 1 measure of perceived public threat:

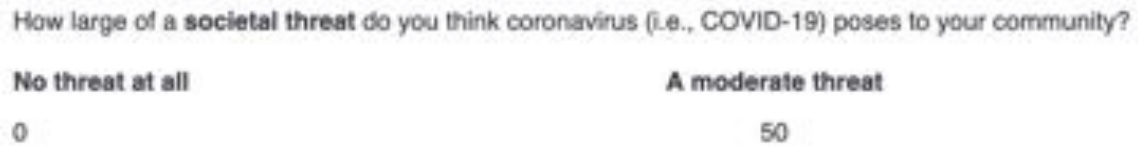

To what extent are you alraid for your community of contracting and spreading coronavirus (i.e., COVID-19)? In other words, to what extent do you fear the collective repercussions for society that would follow from contracting and spreading coronavirus?

Not at all

0
To a moderate extent

50
To an enormous extent

Note that these two pages were measured in random order, but the order of questions on each page was fixed in the order shown. Due to a programming error, this meant that the question starting with "to what extent are you afraid" was shown first when measuring personal threat, and second when measuring public threat. 
Study 2 measure of perceived personal threat:

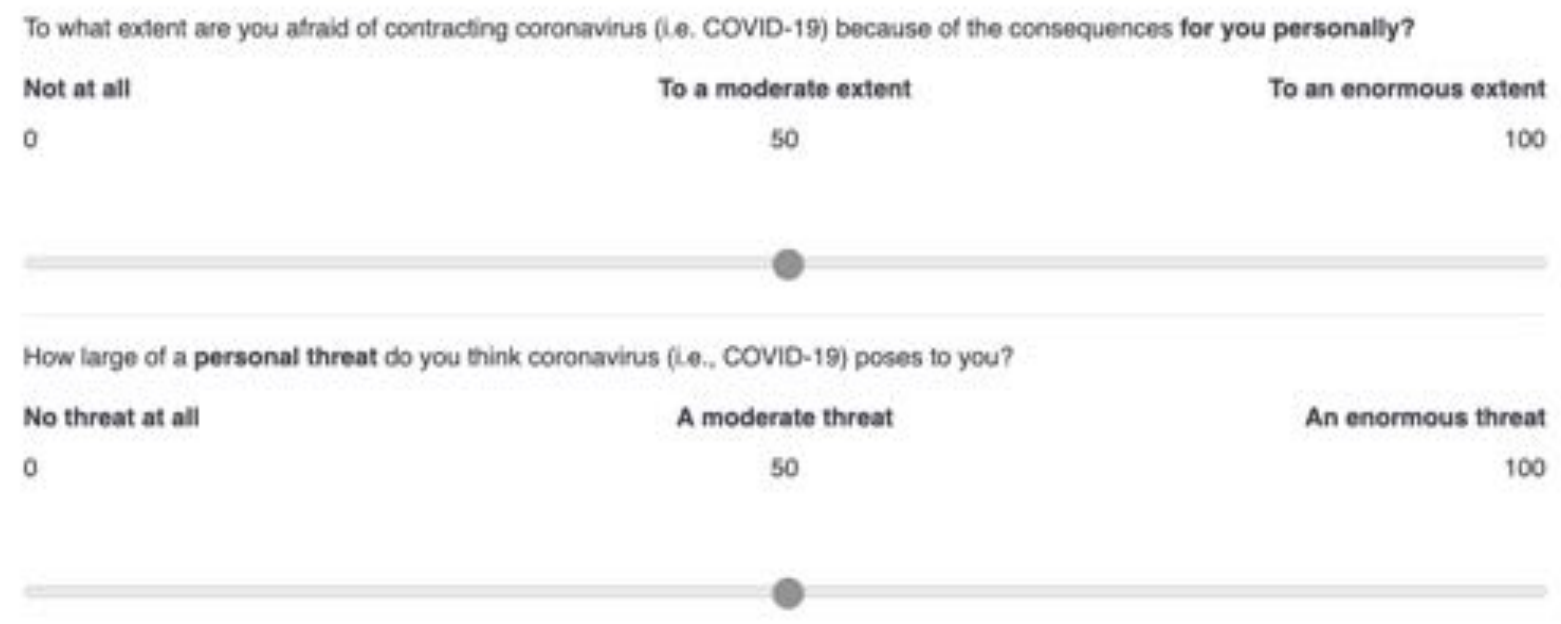

Study 2 measure of perceived public threat:

How large of a collective threat do you think coronavirus (i.e., COVID-19) poses to your community?

No threat at all

A moderate threat

An enormous threat

0

50

100

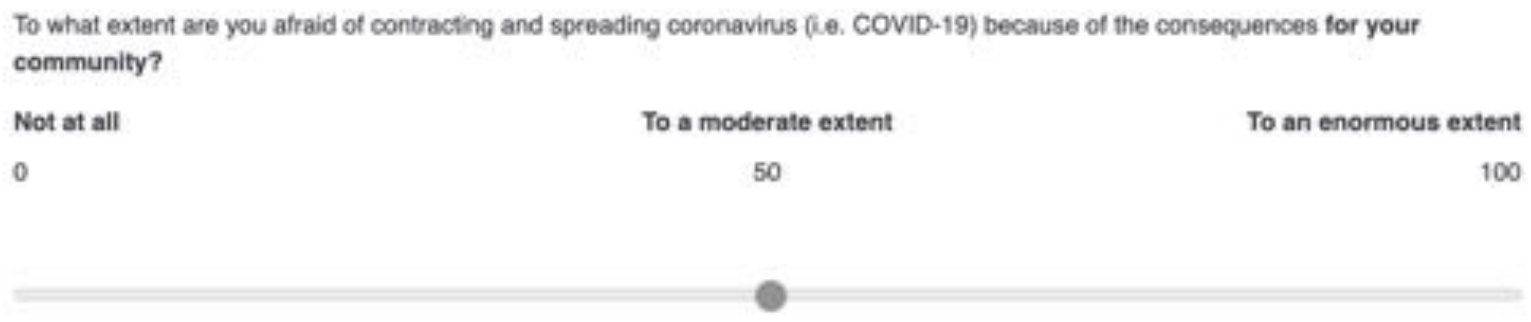

Note that these two pages were measured in random order, but the order of questions on each page was fixed in the order shown. Due to a programming error, this meant that the question starting with "to what extent are you afraid" was shown first when measuring personal threat, and second when measuring public threat.

Study 3 used our Study 2 measures but corrected the aforementioned programming error by randomized the order of questions on each page.

For threat measures in Study 4, see Section 4.9. 


\subsection{Individual difference variables}

Studies 1-2 initial set of questions:

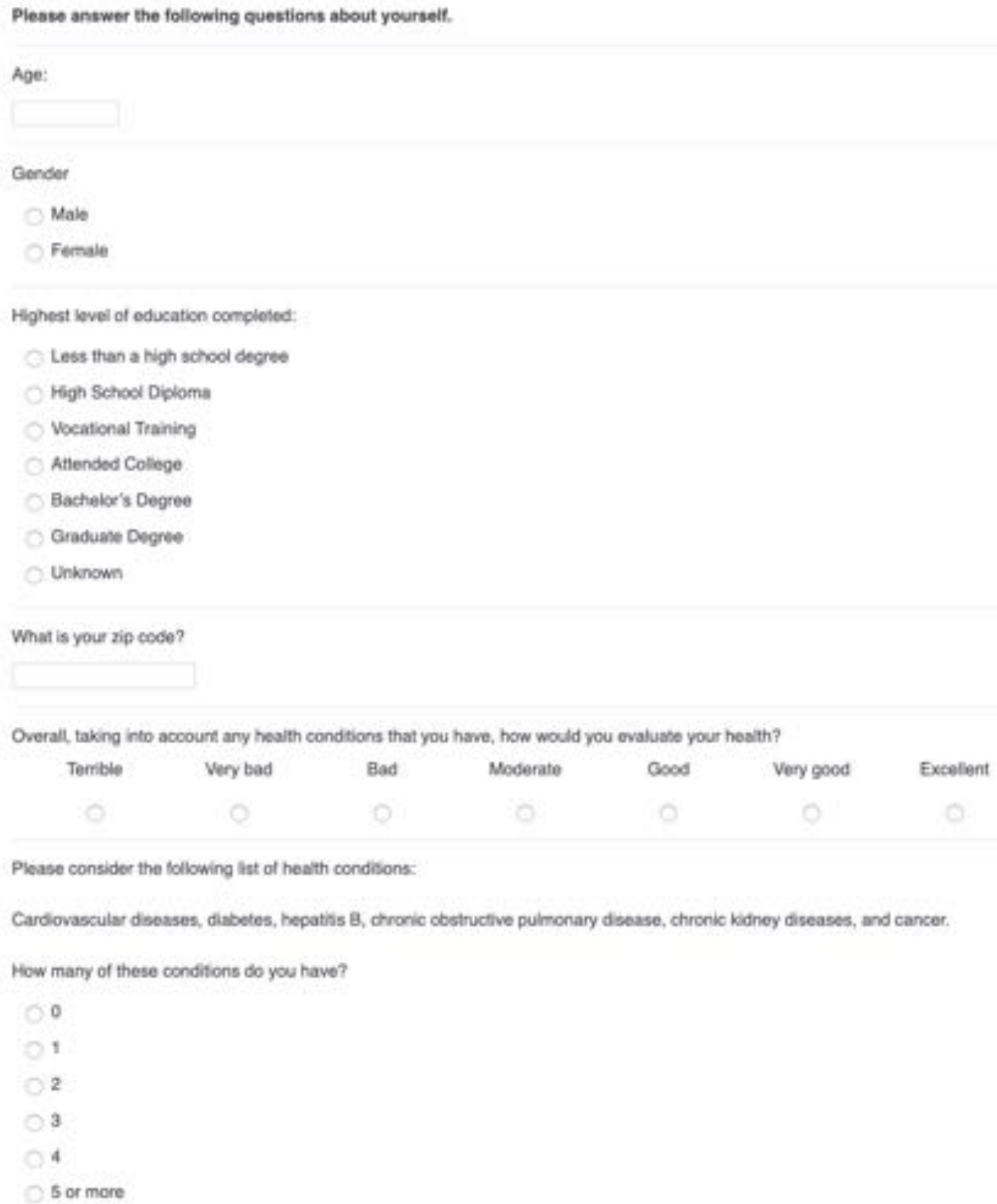




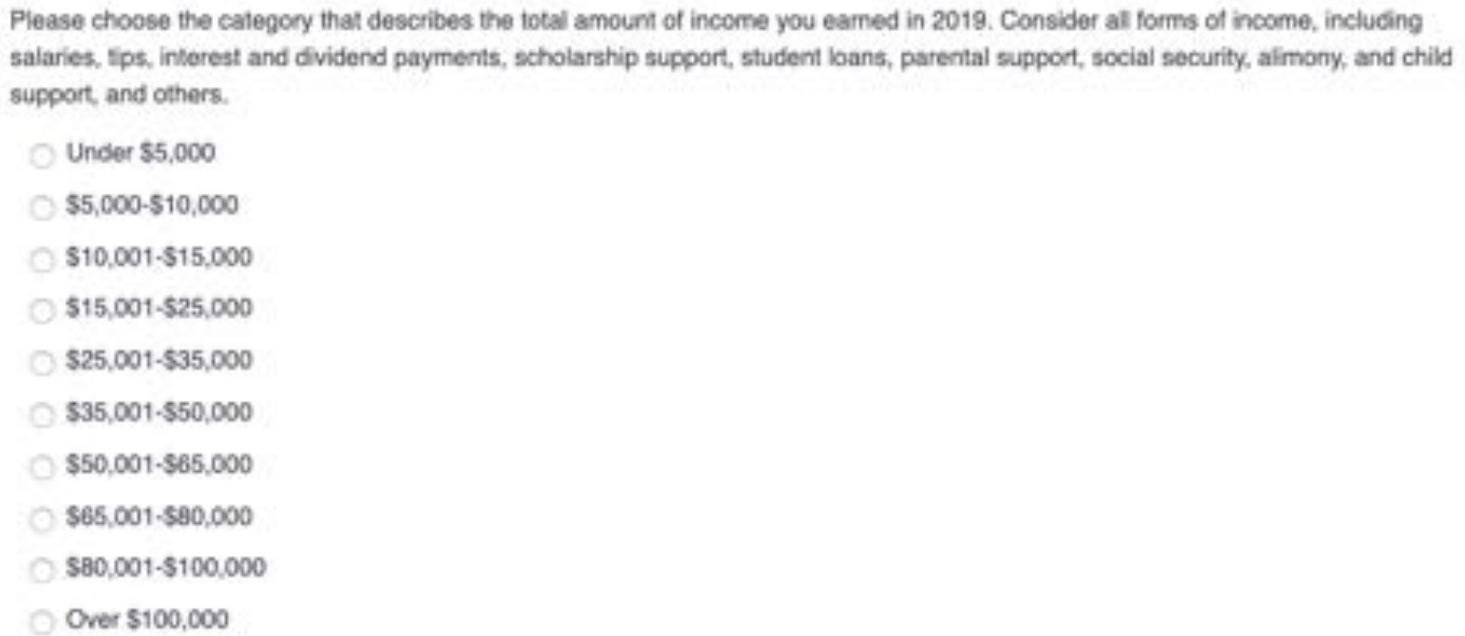

6

7-Strongly

Politically, how conservative are you in terms of social issues?

1 - Very lberal

2

3

4

Politically, how conservative are you in lerms of economic issues?

1 - Very libera:

2

3

4

5

6

7. Vory conservative

Prior to taking this survey, much inlormation have you been exposed to about COVID-9?

Almost none

0

A moderate amount

50
An enormous amount

100

Note that these items were presented in the fixed order shown here. Note also that to compute a "college degree" dummy from our categorical education variable, we coded the data as follows: $0=$ "less than a high school degree", "high school diploma", "vocational training", "attended college", or "unknown"; 1 = "bachelor's degree" or "graduate degree". 
Study 3 included most of these questions, with a few exceptions outlined in Section 1. It also additionally included the two below questions:

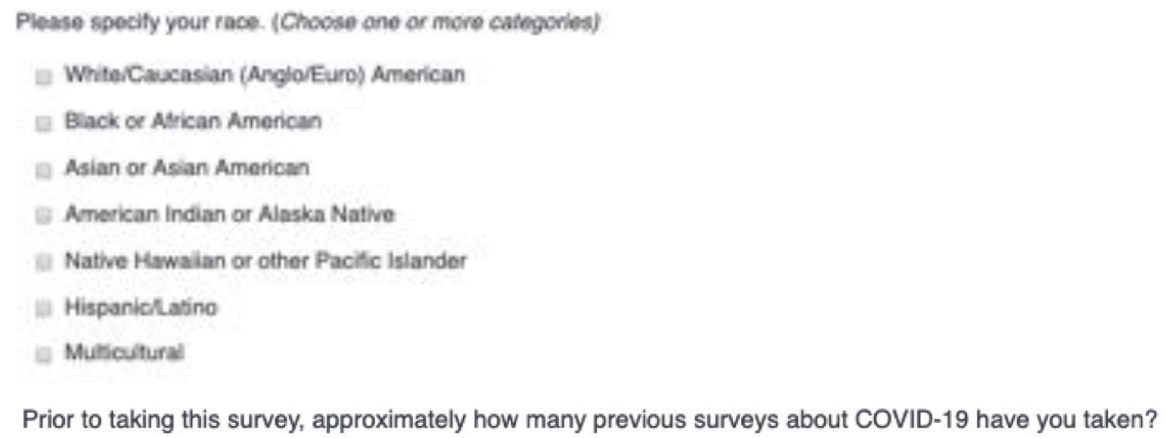

Note that our analyses of race included a series of dummies for each racial category.

Cognitive Reflection Task used in all studies except 3d:

Please do your best to answer the following questions as accurately as possible.

The ages of Mark and Adam add up to 28 years total. Mark is 20 years older than Adam. How many years old is Adam?

If it takes 10 second for 10 printer to print out 10 pages of paper, how many seconds will it take 50 printers to print out 50 pages of paper?

On a loaf of bread, there is a patch of mold. Every day, the patch doubles in size. If it takes 40 days for the patch to cover the entire loaf of bread, how many days would it take for the patch to cover half of the loaf of bread?

How many of the last 3 questions do you think you answered correctly?

Note that each of these four questions was presented on a separate page, in the fixed order shown here. We did not analyze responses to the final question (about the number of questions answered correctly). Correct answers to the first three questions: 4, 10, 39 . 
English check used in Studies 1-2:

Please, now answer a final set of questions.

Dog is to puppy as cat is to

Please write 3 sentences about what you ve done with your day so far, or what you plan to do for the rest of the day.

Attention checks used in Study 3a-c:

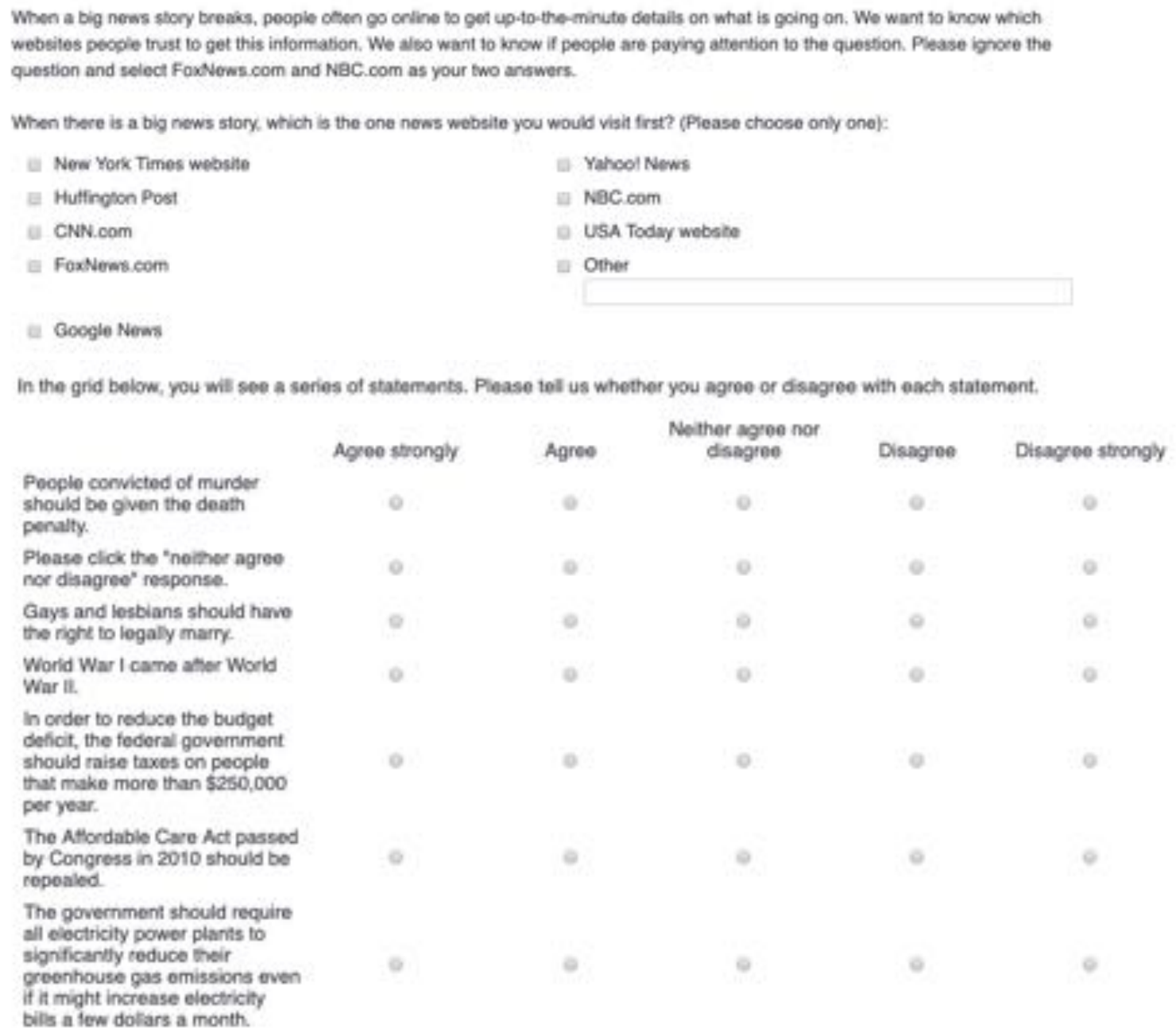

Note that we presented the first attention check after measuring Time 2 prevention intentions (but before measuring the perceived public and personal threat of coronavirus) and we presented the second attention check after measuring all individual difference questions except for the CRT. 


\subsection{Field experiment materials}

The following are the three versions of the materials included in Covid Act Now (CAN)'s daily newsletter. The first version featured our Personal treatment in the header, and was sent to subscribers with last names that began with the letters A through I on December 21, 2021 with the email subject line "A new variant spreading in the U.K.". The header read:

Add your phone to your state's [exposure notification system] to receive alerts when you have been in close contact with someone who later tests positive for COVID. Once you activate the system, you'll need to keep your bluetooth on in order to receive alerts. Your privacy is protected as your identity is not known and your location is not tracked. Participating in the exposure system is one of the most effective actions you can take at the moment. It lets you know if you may have COVID, which helps you obtain treatment more quickly.

The remaining content of that day's newsletter is available at the following url: http://createsend.com/t/t-DCD20D92AB1E0AE12540EF23F30FEDED

The second version featured our Public treatment in the header, and was sent to subscribers with last names that began with the letters J through Q on December 22, 2021 with the email subject line "California hospitals overwhelmed". The header read:

Add your phone to your state's [exposure notification system] to receive alerts when you have been in close contact with someone who later tests positive for COVID. Once you activate the system, you'll need to keep your bluetooth on in order to receive alerts. Your privacy is protected as your identity is not known and your location is not tracked. Participating in the exposure system is one of the most effective actions you can take at the moment. It lets you know if you may have COVID, which prevents you from spreading COVID to more people.

The remaining content of that day's newsletter is available at the following url: http://createsend.com/t/t-7BABFD2072E69B4E2540EF23F30FEDED

The third version featured our Personal + Public treatment in the header, and was sent to subscribers with last names that began with the letters R through Z on December 23, 2021 with the email subject line "100 million more doses". It read:

Add your phone to your state's [exposure notification system] to receive alerts when you have been in close contact with someone who later tests positive for COVID. Once you activate the system, you'll need to keep your bluetooth on in order to receive alerts. Your privacy is protected as your identity is not known and your location is not tracked. Participating in the exposure system is one of the most effective actions you can take at the moment. It lets you know if you may have COVID, which helps you obtain treatment more quickly and prevents you from spreading COVID to more people.

The remaining content of that day's newsletter is available at the following url: http://createsend.com/t/t-CF9B1E0EB93DF96C2540EF23F30FEDED 